\title{
VERTEX ALGEBRAS AND THE FORMAL LOOP SPACE
}

\author{
M. Kapranov AND E. VASSERot
}

\section{INTRODUCTION}

One of the salient mathematical features of string theory is the importance of vertex algebras. Their role in the theory can be compared to that of Lie algebras in the "ordinary" physics of point particles.

Mathematically, the approach of string theory can be cast in terms of analysis on the space of free loops, i.e., smooth maps $S^{1} \rightarrow X$ where $X$ is a given "spacetime" manifold. Accordingly, one has the folklore principle that constructions involving the space of free loops lead to vertex algebras. One class of such constructions is provided by the spaces of highest weight representations of loop groups. Another is $\Omega_{X}^{c h}$, the chiral de Rham complex of an algebraic variety $X$, introduced by Malikov, Schechtman and Vaintrob [MSV]. Heuristically, this complex should be interpreted in terms of $L X$, the space of free loops and its subvariety $L^{0} X$ consisting of loops extending holomorphically into the unit disk. More precisely, $\Omega_{X}^{c h}$ can be thought of as the semiinfinite de Rham complex with coefficients in the space of distributions on $L X$ supported on $L^{0} X$. This is not, however, the way $\Omega_{X}^{c h}$ has been defined mathematically. The definition given in $[\mathrm{MSV}]$ is of more computational nature and proceeds by constructing the action of the group of diffeomorphisms on the irreducible module over the Heisenberg algebra. In that approach it seems miraculous that such an action exists at all.

The aim of this paper is twofold. First, to give a precise mathematical theorem underlying the above folklore principle about vertex algebras. For this, we introduce an algebro-geometric version of the free loop space $\mathcal{L}(X)$ for any scheme $X$ of finite type over a field. This is an ind-scheme containing $\mathcal{L}^{0}(X)$, the scheme of formal germs of curves on $X$ studied in [DL]. We prove that both $\mathcal{L}(X)$ and $\mathcal{L}^{0}(X)$ themselves possess a non-linear version of the vertex algebra structure (which makes it clear that any natural linear construction applied to them should give a vertex 
algebra in the usual sense). More precisely, we use the geometric approach to vertex algebras developed by Beilinson and Drinfeld [BD1] and based on the concepts of chiral algebras and factorization algebras. The latter concept has a natural nonlinear version, that of a factorization monoid. What we prove is that natural "global" versions of $\mathcal{L}(X), \mathcal{L}^{0}(X)$ have natural structures of factorization monoids. An earlier known example of a factorization monoid is given by the affine Grassmannian $[G]$, and this explains why the spaces of representations of loop groups are vertex algebras. Our construction is similar in spirit.

To give a good definition of the algebraic analog of the full loop space $L X$ one has to overcome a certain subtlety. Namely, a natural approach would be to try to (ind-)represent a functor which to any commutative ring $R$ associates the set of $R((t))$-points of $X$. (This is exactly how one defines the scheme $\mathcal{L}^{0}(X)$, with $R[[t]]$ instead of $R((t))$.) If $X$ is affine, this indeed gives a good ind-scheme which we denote $\tilde{\mathcal{L}}(X)$. But when $X$ is, say, projective, then (for $R$ a field) there is no difference between $R[[t]]$-points and $R((t))$-points of $X$ (valuative criterion of properness), so it may seem that nothing is gained by allowing Laurent series. To state this phenomenon differently, the ind-schemes $\tilde{\mathcal{L}}(U)$ for affine $U \subset X$ do not glue together well. This is in fact understandable on general grounds: the loop space $L X$ is not the union of the $L U$ since a loop need not spend all its time in any given $U$.

To get around this difficulty we adopt the following strategy. For an affine $X$ we consider $\mathcal{L}(X)$, the formal neighborhood of $\mathcal{L}^{0}(X)$ in $\tilde{\mathcal{L}}(X)$. So we are dealing with formal loops which are "infinitesimal in the Laurent direction". Then, we prove that the $\mathcal{L}(U), U \subset X$, do indeed possess the right gluing properties. This is due to the infinitesimal nature of our loops.

The role of nilpotent thickenings in Laurent series models for loop spaces was first pointed out by C. Contou-Carrère $[\mathrm{CC}]$ who was studying, in our notation, the group ind-scheme $\tilde{\mathcal{L}}\left(\mathbb{G}_{m}\right)$ and found that it is a nontrivial formal thickening of $\mathcal{L}^{0}\left(\mathbb{G}_{m}\right) \times \mathbb{Z}$

Our second goal is to give a direct geometric construction of $\Omega_{X}^{\text {ch }}$ (for smooth $X$ ) in terms of our model for the loop space. By the above, this construction explains also the fact that $\Omega_{X}^{c h}$ is a sheaf of vertex algebras. In order to achieve this, we represent $\mathcal{L}(X)$ as an ind-pro-object in the category of schemes of finite type and then show that the shifted de Rham complexes of the terms of this ind-pro-system arrange naturally into a double inductive system whose inductive limit is identified with $\Omega_{X}^{c h}$.

As with the study of formal arcs and motivic integration [DL], one can view our considerations as algebro-geometric analogs of the basic constructions of $p$-adic analysis. The difference between our ind-scheme $\mathcal{L}(X)$ and the more familiar scheme 
$\mathcal{L}^{0}(X)$ is similar to the difference between $\mathbb{Q}_{p}$ and $\mathbb{Z}_{p}$ : while the latter is a pro-object in the category of schemes of finite type (resp. finite sets), the former is an ind-proobject. Further, our approach to $\Omega_{X}^{c h}$ is similar to the construction of the space of locally constant functions with compact support on $\mathbb{Q}_{p}=\lim _{i} \lim _{j} p^{-i} \mathbb{Z}_{p} / p^{j} \mathbb{Z}_{p}$ as the double inductive limit of the spaces of functions on the finite sets $p^{-i} \mathbb{Z}_{p} / p^{j} \mathbb{Z}_{p}$, cf $[\mathrm{P}]$. Notice that the reason that these spaces of functions indeed form a double inductive system (with respect to the maps of inverse image in the $j$-direction and direct image in the $i$-direction) is that the commutative squares in the indpro-system $p^{-i} \mathbb{Z}_{p} / p^{j} \mathbb{Z}_{p}$ are Cartesian (so that we have base change). This is an algebraic counterpart of the property of the local compactness of $\mathbb{Q}_{p}$, see [Kat]. In our situation it is equally important that the ind-scheme $\mathcal{L}(X)$ satisfies a certain formal analog of local compactness.

This work has been done over a period of several years during which the first author benefitted from visits to and the financial support of the Université CergyPontoise, the IHES, the Ecole Normale Superieure and the Max-Planck-Institut für Mathematik. His research was also partially supported by grants from NSF and NSERC. The second author is partially supported by EEC grant no. ERB. FMRX-CT97-0100. We would like to thank A. Beilinson for several useful remarks on the earlier version and V. Drinfeld for pointing out the reference [TT].

\section{Construction of the formal loop space}

(2.1) Generalities on schemes and ind-schemes. If $\mathbf{C}$ is a category, then we denote by $\operatorname{Ind}(\mathbf{C})$ and $\operatorname{Pro}(\mathbf{C})$ the categories of ind- and pro-objects of $\mathbf{C}$, see $[\mathrm{AM}][\mathrm{GV}]$ for background. Thus, objects of $\operatorname{Ind}(\mathbf{C})(\operatorname{resp}$. Pro(C)) are symbols "lim ${ }_{i} C_{i}$ (resp. " $\longleftarrow_{i} " C_{i}$ ) where $\left(C_{i}\right)$ is a filtering inductive (resp. projective) system over $\mathbf{C}$, with morphisms defined as in loc. cit. Recall that $\operatorname{Ind}(\mathbf{C})$ can be considered as a full subcategory in $\mathbf{F u n}^{\circ}(\mathbf{C}$, Sets), the category of contravariant functors.

Throughout the paper we fix a field $k$. We denote by Sch $\subset$ Lrs the categories of schemes and locally ringed spaces over $k$. If $R$ is a commutative ring, we will write $\operatorname{Spec} R$ for the topological space (the set of prime ideals with the Zariski topology) underlying the affine scheme Spec $R$ which is thus the ringed space $\left(\underline{\operatorname{Spec}} R, \mathcal{O}_{\text {Spec } R}\right)$.

By an ind-scheme we will mean in this paper an ind-object of $\mathbf{S c h}$ represented by an inductive system of closed embeddings of quasi-compact schemes. The category of ind-schemes will be denoted by Isch. Let us make the category Sch into a Grothendieck site by using Zariski open coverings and let Shf be the category of 
sheaves of sets on Sch. For any ind-scheme $Y$ the functor $\eta_{Y}$ on schemes represented by $Y$ is then a sheaf, so we have the embeddings

$$
\operatorname{Sch} \subset \operatorname{Isch} \subset \operatorname{Shf} \subset \operatorname{Fun}^{\circ}(\operatorname{Sch}, \text { Sets })
$$

Since the category $\mathbf{S c h}$ has finite inverse limits, so do all the categories in (2.1.1) and the embeddings preserve them. On the contrary, finite direct limits, such as cokernels (when they exist) are preserved by the first two of the embeddings but not by the third one: cokernels in the category of sheaves are not the same as in the category of all functors (presheaves).

We denote by $\mathbf{A l g}$ the category of $k$-algebras and by $\mathbf{A f f} \subset \mathbf{S c h}$ the dual category of affine schemes. Note that Isch can be as well realized as a full subcategory in Fun $^{\circ}($ Aff, Sets $)=$ Fun $($ Alg, Sets $)$.

Given two contravariant functors $\phi, \phi^{\prime}: \mathbf{S c h} \rightarrow$ Sets, and a morphism $F: \phi^{\prime} \rightarrow$ $\phi$, we will say that $F$ is formally smooth (resp. formally étale), if for any nilpotent extension of affine schemes $S \subset S^{\prime}$ the natural map

$$
\phi^{\prime}\left(S^{\prime}\right) \rightarrow \phi\left(S^{\prime}\right) \times_{\phi(S)} \phi^{\prime}(S)
$$

is surjective (resp. bijective).

We will say that $F$ is an open embedding, if for any scheme $S$ and any $u \in \phi(S)$ (which is the same as a morphism $\eta_{S} \rightarrow \phi$ ) the fiber product functor $\eta_{S} \times_{\phi} \phi^{\prime}$ is representable by a scheme $S^{\prime}$ whose natural morphism to $S$ is an open embedding.

We define formal smoothness and openness for morphisms of ind-schemes by considering their representable functors.

For a scheme $Z$ we denote by $Z_{\text {red }} \subset Z$ the corresponding reduced subscheme. We extend this notation to ind-schemes by applying it term by term in inductive systems.

Let $X$ be a $k$-scheme of finite type. We denote by $\mathbf{A f f}_{X}\left(\right.$ resp. $\mathbf{A f f}_{X}^{\mathrm{ft}}$ ) the category of schemes affine over $X$ (resp. affine of finite type over $X$ ). For future use let us quote the following fact [EGAIV, Corollary 8.13.2].

(2.1.2) Proposition. The category $\operatorname{Pro}\left(\mathbf{A f f}_{X}^{\mathrm{ft}}\right)$ is equivalent to $\mathbf{A f f}_{X}$ via the functor " $\longleftarrow_{n} " S_{n} \mapsto \lim _{n} S_{n}$.

We also denote $\mathbf{S c h}_{X}$ the category of all $X$-schemes and $\mathbf{I s c h}_{X}$ the category of ind-schemes over $X$. Thus objects of $\mathbf{I s c h}_{X}$ are arrows $Y \rightarrow X, Y \in \mathbf{I s c h}$, or, equivalently, symbols "lim " $Y_{n}$ where $Y_{n} \rightarrow X$ form an inductive system of closed embeddings of quasicompact $X$-schemes.

\section{(2.2) The scheme of germs of arcs.}


Let $X$ be a scheme. We denote by $\mathcal{L}^{0}(X)$ the scheme of germs of $\operatorname{arcs}$ on $X$, see [DL] [BLR, Theorem 7.6.4]. It represents the following covariant functor $\lambda_{X}^{0}$ on the category Alg:

$$
\lambda_{X}^{0}: R \mapsto \operatorname{Hom}_{\mathbf{S c h}}(\operatorname{Spec} R[[t]], X) .
$$

Here are some of the well-known properties of $\mathcal{L}^{0}(X)$. Note that if $R$ is a local ring with maximal ideal $M$, then $R[[t]]$ is a local ring with maximal ideal $M[[t]]+t R[[t]]$.

(2.2.1) Proposition. (a) For any scheme $S$ the pair $\left(S, \mathcal{O}_{S}[[t]]\right)$ is a locally ringed space.

(b) For any scheme $S$ we have

$$
\operatorname{Hom}_{\operatorname{Sch}}\left(S, \mathcal{L}^{0}(X)\right)=\operatorname{Hom}_{\mathbf{L r s}}\left(\left(S, \mathcal{O}_{S}[[t]]\right), X\right)
$$

(c) The scheme $\mathcal{L}^{0}(X)$ is the projective limit of the schemes $\mathcal{L}_{n}^{0}(X), n \in \mathbb{N}$, representing the functors

$$
\lambda_{n, X}^{0}: R \mapsto \operatorname{Hom}_{\mathbf{S c h}}\left(\operatorname{Spec} R[t] / t^{n+1}, X\right) .
$$

If $X$ is of finite type, than so is each $\mathcal{L}_{n}^{0}(X)$.

(d) Denote $\pi_{n}: \mathcal{L}_{n}^{0}(X) \rightarrow X, \pi: \mathcal{L}^{0}(X) \rightarrow X$ the natural projections. They are affine morphisms. For an open subset $U \subset X$ we have $\pi^{-1}(U)=\mathcal{L}^{0}(U)$ and $\pi_{n}^{-1}(U)=\mathcal{L}_{n}^{0}(U)$.

(e) If $X$ is smooth, then so is $\mathcal{L}_{n}^{0}(X)$ and $\mathcal{L}^{0}(X)$ is formally smooth.

Proof: For (a) and (b) it suffices to assume that $S=\operatorname{Spec} R$ is affine. The embedding of constant series and the evaluation at 0 give ring homomorphisms $R \stackrel{\alpha}{\rightarrow} R[[t] \stackrel{\stackrel{\beta}{\rightarrow}}{\rightarrow}$ and hence morphisms of topological spaces

$$
\underline{\operatorname{Spec}} R \stackrel{p}{\longleftarrow} \underline{\operatorname{Spec}} R[[t]] \stackrel{i}{\longleftarrow} \underline{\operatorname{Spec}} R .
$$

The statement (a) follows from the next lemma, since $\mathcal{O}_{\text {Spec }} R[[t]]$ is obviously a sheaf of local rings.

(2.2.3) Lemma. We have

$$
i^{-1} \mathcal{O}_{\text {Spec } R[[t]]}=\mathcal{O}_{\text {Spec } R}[[t]]=p_{*} \mathcal{O}_{\text {Spec } R[[t]]} .
$$

Proof: Let us prove the first equality. If $\mathfrak{p} \in \operatorname{Spec} R$ is a prime ideal in $R$, then the stalk of $i^{-1} \mathcal{O}_{\text {Spec } R[[t]]}$ at $\mathfrak{p}$ is the localization of $R[[t]]$ with respect to the multiplicative subset $\beta^{-1}(R-\mathfrak{p})$ while the stalk of $\mathcal{O}_{\text {Spec } R}[[t]]$ is $R[[t]] \otimes_{R} R_{\mathfrak{p}}$ where $R_{\mathfrak{p}}$ denotes, as usual, the localization of $R$ with respect to $R-\mathfrak{p}$. Now, to see that the two rings are the same, it suffices to use the following obvious property of 
formal power series rings: if $A$ is a commutative ring and $f(t) \in A[[t]]$ is such that $f(0)$ is invertible in $A$, then $f(t)$ is invertible in $A[[t]]$.

The second equality is obvious: the stalk of $p_{*} \mathcal{O}_{\text {Spec } R[[t]]}$ at $\mathfrak{p}$ is immediately seen to coincide with $R[[t]] \otimes_{R} R_{\mathfrak{p}}$.

Now, composing with $i$ defines a map of sets

$$
\phi: \operatorname{Hom}_{\mathbf{S c h}}(\operatorname{Spec} R[[t]], X) \rightarrow \operatorname{Hom}_{\mathbf{L r s}}\left(\left(\operatorname{Spec} R, \mathcal{O}_{\text {Spec } R}[[t]]\right), X\right) .
$$

A map $\psi$ in the other direction comes from the second equality in (2.2.3). One verifies easily that $\phi$ and $\psi$ are mutually inverse. This concludes the proof of (2.2.1)(b). The rest of (2.2.1) is proved in loc. cit.

We will also need the following generalization of Proposition 2.2.1(d).

(2.2.4) Proposition. Let $\phi: X \rightarrow Y$ be an étale morphism of schemes. Then

(a) each morphism $\mathcal{L}_{n}^{0}(\phi): \mathcal{L}_{n}^{0}(X) \rightarrow \mathcal{L}_{n}^{0}(Y)$ is étale,

(b) the square

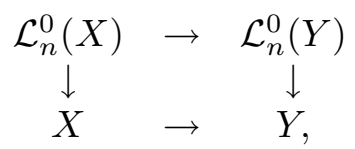

as well as the analogous square for $\mathcal{L}^{0}(X), \mathcal{L}^{0}(Y)$, is Cartesian.

Proof: We can assume that $X=\operatorname{Spec}(A), Y=\operatorname{Spec}(B)$ are affine. It is enough to prove that for each $n \geq 0$ the natural morphism

$$
\alpha: \mathcal{L}_{n}^{0}(X) \rightarrow \mathcal{L}_{n}^{0}(Y) \times_{Y} X
$$

is an isomorphism. Let us construct the inverse morphism $\beta$. Let $R$ be a $k$-algebra and $f$ be a morphism $S \rightarrow \mathcal{L}_{n}^{0}(Y) \times_{Y} X$. Thus, $f$ corresponds to a pair of ring homomorphisms forming the horizontal arrows of the commutative diagram

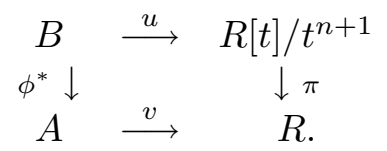

Here $\pi$ is the natural projection. Since $\phi$ is étale and $\pi$ is nilpotent, there is a unique homomorphism $w: A \rightarrow R[t] / t^{n+1}$ such that both resulting triangles are commutative. Let $g$ be the morphism $S \rightarrow \mathcal{L}_{n}^{0}(X)$ represented by $w$. Then we set $\beta(f)=g$. The verifications are obvious and left to the reader.

(2.3) Nil-Laurent series. Let $R$ be a commutative ring. A nil-Laurent series is, by definition, a Laurent series $a(t)=\sum_{i \gg-\infty} a_{i} t^{i} \in R((t))$ such that all the $a_{i}$ with $i<0$ are nilpotent. The set of such series will be denoted $R((t)) \sqrt{ }$.

It is clear that $R((t))^{\sqrt{ }}$ is a subring in $R((t))$, the ring of all Laurent series. Indeed, let $\sqrt{R}$ be the radical of $R$ (the set of all nilpotent elements) and set $R_{\text {red }}=R / \sqrt{R}$. Consider the homomorphism $\rho: R((t)) \rightarrow R_{\text {red }}((t))$ induced by the projection $R \rightarrow R_{\text {red }}$. Then $R((t))^{\sqrt{ }}=\rho^{-1}\left(R_{\text {red }}[[t]]\right)$. 
(2.3.1) Proposition. For $a=\sum a_{i} t^{i} \in R((t))^{\sqrt{ }}$ the following are equivalent:

(i) the element a is invertible in $R((t)) \sqrt{ }$,

(ii) the element $\rho(a)$ is invertible in $R_{\text {red }}[[t]]$,

(iii) the element $a_{0}$ is invertible in $R$.

Proof: (i) $\Rightarrow$ (ii) is obvious. To see that (ii) $\Leftrightarrow($ iii), note that the invertibility of $\rho(a)$ implies the invertibility of the image of $a_{0}$ in $R_{\text {red }}$ which certainly implies the invertibility of $a_{0}$ and the converse is equally obvious. Let us prove that (ii) $\Rightarrow(\mathrm{i}$ ). Indeed, if $\rho(a)$ is invertible in $R_{\text {red }}[[t]]$, we have $a b=1+c$ for some $b \in R((t))^{\sqrt{ }}$, $c \in \operatorname{Ker} \rho=\sqrt{R}((t))$. But every element $c \in \sqrt{R}((t))$ is topologically nilpotent (setting $c=c_{-}+c_{+}$with $c_{-} \in t^{-1} \sqrt{R}\left[t^{-1}\right]$ and $\left.c_{+} \in \sqrt{R}[t]\right]$, we have that $c_{-}$is nilpotent while $c_{+}$is topologically nilpotent), thus $a b$ is invertible in $R((t))^{\sqrt{ }}$, and $a$ is invertible, too.

(2.3.2) Corollary. If $R$ is a local ring with maximal ideal $M$, then $R((t))^{\sqrt{ }}$ is a local ring with maximal ideal $\rho^{-1}\left(M_{\text {red }}[[t]]+t R_{\text {red }}[[t]]\right)$.

(2.4) The formal loop space. We now describe our main construction. Let $X$ be a scheme of finite type over $k$. Define a covariant functor $\lambda_{X}$ from Alg to sets as follows:

$$
\lambda_{X}(R)=\operatorname{Hom}_{\mathbf{S c h}}\left(\operatorname{Spec} R((t))^{\sqrt{ }}, X\right) .
$$

(2.4.2) Theorem. (a) The functor $\lambda_{X}$ is represented by an ind-scheme $\mathcal{L}(X)$, containing $\mathcal{L}^{0}(X)$.

(b) $\mathcal{L}(X)$ is an inductive limit of nilpotent extensions of $\mathcal{L}^{0}(X)$. In particular, for any open set $Y \subset \mathcal{L}^{0}(X)$ there is a well-defined ind-scheme $\left.\mathcal{L}(X)\right|_{Y}$.

(c) If $U$ is an open subset in $X$, then the ind-scheme $\mathcal{L}(U)$ is identified with $\left.\mathcal{L}(X)\right|_{\pi^{-1}(U)}$.

(d) If $X$ is smooth, then $\mathcal{L}(X)$ is formally smooth.

Note that Theorem 2.4.2 is closely related to [BD1, Proposition 3.9.3.(i)]. The proof will be finished in the next subsection.

Let $R$ be a commutative ring. Since $R((t))^{\sqrt{ }}$ is a nilpotent extension of $R[[t]]$, we have $\operatorname{Spec} R((t))^{\sqrt{ }}=\operatorname{Spec} R[[t]]$, so Spec $R((t))^{\sqrt{ }}$ is the ringed space formed by $\underline{\text { Spec }} R[[t]]$ and a certain sheaf of rings $\mathcal{O}_{\text {Spec } R((t))^{v}}$ on it.

(2.4.4) Lemma. (a) We have, with respect to the maps in (2.2.2), the equalities

$$
i^{-1} \mathcal{O}_{\text {Spec } R((t))^{\mathcal{V}}}=\mathcal{O}_{\text {Spec } R}((t))^{\sqrt{ }}=p_{*} \mathcal{O}_{\text {Spec } R((t))^{\mathcal{V}}}
$$

(b) For any scheme $S$ the sheaf $\mathcal{O}_{S}((t))^{\sqrt{ }}$ is a sheaf of local rings 
The proof of (a) is analogous to that of Lemma 2.2.3. Instead of the property of $A[[t]]$ quoted there, we use Proposition 2.3.1. Part (b) follows from (a).

Let $\psi: \mathbf{S c h} \rightarrow$ Lrs be the functor such that $S \mapsto\left(S, \mathcal{O}_{S}((t))^{\sqrt{ }}\right)$. Let us define a contravariant functor $\lambda_{X}^{\prime}$ on the category $\mathbf{S c h}$ by

$$
\lambda_{X}^{\prime}(S)=\operatorname{Hom}_{\mathbf{L r s}}(\psi(S), X) .
$$

(2.4.5) Proposition. For an affine scheme $S=\operatorname{Spec}(R)$ we have $\lambda_{X}^{\prime}(S)=$ $\lambda_{X}(R)$.

Proof: Follows from Lemma 2.4.4 similarly to Proposition 2.2.1(a).

In virtue of Proposition 2.4.5, for the proof of Theorem 2.4.2 it suffices to show that the functor $\lambda_{X}^{\prime}$ on $\mathbf{S c h}$ is ind-representable. We start by estabilishing some of its properties.

(2.4.6) Proposition. (a) For every scheme $X$ the functor $\lambda_{X}^{\prime}$ is a sheaf on $\mathbf{S c h}$.

(b) If $U \subset X$ is an open subset, then the induced morphism of functors $\lambda_{U}^{\prime} \rightarrow \lambda_{X}^{\prime}$ is open.

(c) Let $\left(U_{\alpha}\right)_{\alpha \in A}$ be an open covering of $X$. Then $\lambda_{X}^{\prime}$ is equal to the cokernel, in the category Shf, of the pair of morphisms

$$
\coprod_{\alpha, \beta} \lambda_{U_{\alpha} \cap U_{\beta}}^{\prime} \rightrightarrows \coprod_{\alpha} \lambda_{U_{\alpha}}^{\prime}
$$

Proof: The proposition follows from simple properties of representable functors on the category Lrs. If $\mathcal{T}=\left(T, \mathcal{O}_{\mathcal{T}}\right)$ is a locally ringed space, we will call an open part of $\mathcal{T}$ a ringed space of the form $\left(U,\left.\mathcal{O}_{\mathcal{T}}\right|_{U}\right)$ where $U \subset T$ is an open subset in the usual sense. An open embedding is, by definition, a morphism isomorphic to the inclusion of an open part. Accordingly, we have the concept of an open covering of $\mathcal{T}$. This makes Lrs into a Grothendieck site. For $\mathcal{X} \in \operatorname{Lrs}$ let $\eta_{\mathcal{X}}$ be the corresponding representable functor on Lrs. As in (2.1), a morphism $F: \phi^{\prime} \rightarrow \phi$ of functors Lrs $\rightarrow$ Sets will be called open, if for any $\mathcal{S} \in$ Lrs and any element $u \in \phi(\mathcal{S})$ (i.e., a morphism $\eta_{\mathcal{S}} \rightarrow \phi$ ) the fiber product functor $\eta_{\mathcal{S}} \times_{\phi} \phi^{\prime}$ is representable by a locally ringed space $\mathcal{S}^{\prime}$ whose natural morphism to $\mathcal{S}$ is an open embedding. Let us recall the following basic facts.

(2.4.7) Lemma. (a) For any $\mathcal{X} \in$ Lrs the representable functor $\eta_{\mathcal{X}}$ is a sheaf on Lrs.

(b) If $\mathcal{U} \subset \mathcal{X}$ is an open embedding in Lrs, then $\eta_{\mathcal{U}} \rightarrow \eta_{\mathcal{X}}$ is an open morphism of functors.

(c) Let $\mathcal{X} \in \operatorname{Lrs}$ and $\left(\mathcal{U}_{\alpha}\right)_{\alpha \in A}$ be an open covering of $\mathcal{X}$. Then in the category of sheaves of sets on $\mathbf{L r s}$ we have the equality

$$
\eta_{\mathcal{X}}=\operatorname{Coker}\left\{\coprod_{\alpha, \beta} \eta_{\mathcal{U}_{\alpha} \cap \mathcal{U}_{\beta}} \rightrightarrows \coprod_{\alpha} \eta_{\mathcal{U}_{\alpha}}\right\}
$$


or, explicitly, for any $\mathcal{S} \in \mathbf{L r s}$,

$$
\eta_{\mathcal{X}}(\mathcal{S})=\lim _{\overrightarrow{\mathcal{S}_{\alpha}}} \operatorname{Ker}\left\{\prod_{\alpha} \eta_{\mathcal{U}_{\alpha}}\left(\mathcal{S}_{\alpha}\right) \rightrightarrows \prod_{\alpha \beta} \eta_{\mathcal{U}_{\alpha} \cap \mathcal{U}_{\beta}}\left(\mathcal{S}_{\alpha} \cap \mathcal{S}_{\beta}\right)\right\}
$$

where the limit is taken over the set of open coverings $\left(\mathcal{S}_{\alpha}\right)_{\alpha \in A}$ of $\mathcal{S}$ (the indexing set $A$ being fixed) ordered by simultaneous inclusion.

Proposition 2.4.6 follows from Lemma 2.4.7. Indeed, we have $\lambda_{X}^{\prime}=\eta_{X} \circ \psi$, where $X$ is viewed as a locally ringed space. Thus, to prove Proposition 2.4.6.(b) using Lemma 2.4.7.(b) it is enough to prove that for any open embedding of schemes $U \hookrightarrow X$ and any $u \in \lambda_{X}^{\prime}(S)$ there is an open embedding $j: S^{\prime} \hookrightarrow S$ such that the following square is Cartesian

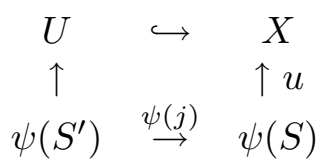

(then, $\eta_{S} \times_{\lambda_{X}^{\prime}} \lambda_{U}^{\prime}=\eta_{S^{\prime}}$ ). This is obvious.

Proof of (2.4.7) : Part (a) is obvious, and Part (b) is proved in [EGA0, (4.5.2)]. Let us prove Part $(c)$. For any $\phi \in \eta_{\mathcal{X}}(\mathcal{S})$ and any $\alpha$, consider the ringed space $\mathcal{S}_{\alpha}=\left(\phi^{-1}\left(\mathcal{U}_{\alpha}\right),\left.\mathcal{O}_{\mathcal{S}}\right|_{\phi^{-1}\left(\mathcal{U}_{\alpha}\right)}\right)$. Clearly, $\left(\mathcal{S}_{\alpha}\right)$ is an open covering of $\mathcal{S}$. Let $\phi_{\alpha}$ be the restriction of $\phi$ to $\mathcal{S}_{\alpha}$. Then, $\left(\phi_{\alpha}\right)$ is an element of $\prod_{\alpha} \eta_{\mathcal{U}_{\alpha}}\left(\mathcal{S}_{\alpha}\right)$ lying in the kernel (2.4.9). Thus we have constructed a map from $\eta_{\mathcal{X}}(\mathcal{S})$ to the right hand side of (2.4.9). On the other hand, assume that $\left(\phi_{\alpha}\right) \in \prod_{\alpha} \eta_{\mathcal{U}_{\alpha}}\left(\mathcal{S}_{\alpha}\right)$ is such that $\phi_{\alpha}\left(\mathcal{S}_{\alpha} \cap \mathcal{S}_{\beta}\right) \subset \mathcal{U}_{\alpha} \cap \mathcal{U}_{\beta}$ and $\left.\phi_{\alpha}\right|_{\mathcal{S}_{\alpha} \cap \mathcal{S}_{\beta}}=\left.\phi_{\beta}\right|_{\mathcal{S}_{\alpha} \cap \mathcal{S}_{\beta}}$. The corresponding maps $\mathcal{S}_{\alpha, \text { red }} \rightarrow$ $\mathcal{U}_{\alpha, \text { red }}$ glue together, giving a continuous map $\phi_{\text {red }}: \mathcal{S}_{\text {red }} \rightarrow \mathcal{X}_{\text {red }}$. Moreover, there is an obvious sheaf homomorphism $\phi^{*}: \phi_{\text {red }}^{-1} \mathcal{O}_{\mathcal{X}} \rightarrow \mathcal{O}_{\mathcal{S}}:$ the restriction of $\phi^{*}$ to $\mathcal{S}_{\alpha}$ is the composition of the chain of maps

$$
\left.\left(\phi_{\text {red }}^{-1} \mathcal{O}_{\mathcal{X}}\right)\right|_{\mathcal{S}_{\alpha}}=\left.\left(\phi_{\alpha, \text { red }}^{-1} \mathcal{O}_{\mathcal{U}_{\alpha}}\right)\right|_{\mathcal{S}_{\alpha}} \stackrel{\phi_{\alpha}^{*}}{\longrightarrow} \mathcal{O}_{\mathcal{S}_{\alpha}}=\left.\mathcal{O}_{\mathcal{S}}\right|_{\mathcal{S}_{\alpha}}
$$

This establishes (2.4.9).

Let $C$ be the cokernel as in (2.4.8) but taken in the category of presheaves. Then the cokernel in the category of sheaves is just the sheaf $\bar{C}$ associated to the presheaf $C$. By definition, for $\mathcal{S} \in \operatorname{Lrs}$ the set $C(\mathcal{S})$ consists of pairs $\left(\alpha, \phi: S \rightarrow \mathcal{U}_{\alpha}\right)$ taken modulo the identifications coming from morphisms of $\mathcal{S}$ into $\mathcal{U}_{\alpha} \cap \mathcal{U}_{\beta}$. Now, by the definition of the sheaf associated to the presheaf,

$$
\bar{C}=\lim _{\longrightarrow \mathcal{S}=\cup_{\gamma \in \Gamma} \mathcal{S}_{\gamma}} \operatorname{Ker}\left\{\prod_{\gamma \in \Gamma} C\left(\mathcal{S}_{\gamma}\right) \rightarrow \prod_{\gamma, \gamma^{\prime} \in \Gamma} C\left(\mathcal{S}_{\gamma} \cap \mathcal{S}_{\gamma^{\prime}}\right)\right\},
$$


where the limit is over all possible open coverings of $\mathcal{S}$ (with arbitrary indexing sets $\Gamma$ ) ordered by refinement. Notice now that specifying a section of $C$ over $\mathcal{S}_{\gamma}$ includes specifying an index $\alpha$ from the set $A$ indexing the covering $\left\{\mathcal{U}_{\alpha}\right\}$, so we get a map $p: \Gamma \rightarrow A$. Denoting $\mathcal{S}_{\alpha}=\bigcup_{p(\gamma)=\alpha} \mathcal{S}_{\gamma}$, we get an element of the right hand side of (2.4.9). This establishes the equivalence of (2.4.8) and (2.4.9).

Note that Proposition 2.4.6(c) implies the following.

(2.4.10) Corollary. We have $\lambda_{X}=\lim _{U \subset X \text { affine }} \lambda_{U}$, the limit being taken in the category Shf.

(2.5) Proof of Theorem 2.4.2. We first assume that $X=\operatorname{Spec} A$ is an affine scheme of finite type. Consider the larger functor $\tilde{\lambda}_{X}$ on Alg defined by

$$
\tilde{\lambda}_{X}(R)=\operatorname{Hom}_{\mathbf{A l g}}(A, R((t))) .
$$

(2.5.1) Proposition. (a) The functor $\tilde{\lambda}_{X}$ is represented by an ind-scheme $\tilde{\mathcal{L}}(X)$, which is an inductive limit of affine schemes of infinite type.

(b) If $X$ is smooth, then $\tilde{\mathcal{L}}(X)$ is formally smooth.

Proof: (a) Consider the $k$-ind-scheme

$$
\underline{k((t))}=\underline{\lim }_{N} \operatorname{Spec} k\left[a_{l} ;-N \leq l\right] .
$$

We can think of the $a_{i}$ as the coefficients of an indeterminate Laurent series $\sum a_{i} t^{i}$. It is clear that $k((t))$ represents the functor $\tilde{\lambda}_{\mathbb{A}^{1}}$. Since $\mathbb{A}^{1}$ is a $k$-algebra object in the category of schemes, multiplication of Laurent series makes $\underline{k((t))}$ into a $k$ algebra object in the category of ind-schemes. In particular, each polynomial $f \in$ $k\left[x_{1}, \ldots, x_{d}\right]$ defines a morphism of ind-schemes $\tilde{\mathcal{L}}(f): \underline{k((t))^{d}} \rightarrow \underline{k((t))}$. Hereafter we will write $k[x]$ instead of $k\left[x_{1}, \ldots, x_{d}\right]$ to simplify.

Suppose now that $X$ is given in $\mathbb{A}^{d}$ by a system of algebraic equations, say

$$
f_{m}\left(x_{1}, \ldots, x_{d}\right)=0, \quad m=1,2, \ldots, l .
$$

The ind-scheme $\tilde{\mathcal{L}}(X)$ is realized as the closed sub-ind-scheme in $\underline{k((t))}^{d}$ defined as the intersection of the preimages of 0 under the $\tilde{\mathcal{L}}\left(f_{m}\right)$. More explicitly, replacing $x_{i}$ by $x_{i}(t)=\sum_{j \geq-N} a_{j}^{(i)} t^{j}$ in the equations above, we get, for each $N$, a system of algebraic equations in $k\left[a_{l}^{(i)} ;-N \leq l\right]$ for each $N$ which defines a subscheme in $\left(\operatorname{Spec} k\left[a_{l} ;-N \leq l\right]\right)^{d}$. Our desired ind-scheme $\tilde{\mathcal{L}}(X)$ is the direct limit of these schemes as $N \rightarrow \infty$.

(b) The infinitesimal lifting condition for $\tilde{\lambda}_{X}$ is formulated for affine schemes $S=\operatorname{Spec}(R)$. We need to prove that for any surjection of $k$-algebras $R^{\prime} \rightarrow R$ 
whose kernel $I$ satisfies $I^{n}=0$ for some $n$, the map of sets $\tilde{\lambda}_{X}\left(R^{\prime}\right) \rightarrow \tilde{\lambda}_{X}(R)$ is surjective. But the kernel of $R^{\prime}((t)) \rightarrow R((t))$ is $I((t))$ which is also nilpotent of order $n$. So the smoothness of $A$ implies that any morphism $A \rightarrow R((t))$ can be lifted to a morphism $A \rightarrow R^{\prime}((t))$.

(2.5.2) Corollary. The functor $\lambda_{X}$ is represented by an ind-scheme $\mathcal{L}(X)$ which is the inductive limit of the formal neighborhoods of $\mathcal{L}^{0}(X)$ in the schemes forming an inductive system for $\tilde{\mathcal{L}}(X)$. If $X$ is smooth, then $\mathcal{L}(X)$ is formally smooth.

This proves parts (a), (b) and (d) of Theorem 2.4.2 for the case of affine $X$. Part (c) of the theorem follows from Proposition 2.4.6(c).

To prove Theorem 2.4.2 for general $X$, it is enough to establish the existence of the limit $\lim _{U \subset X \text { affine }} \mathcal{L}(U)$ in the category of ind-schemes. In fact, it is enough to take the limit over a finite set of $U$ consisting of elements of some finite covering and their intersections. Indeed, given this, all the other properties follow from the affine case and from Proposition 2.4.6. But using Proposition 2.4.6(b) again, we see that for any pair $U^{\prime} \subset U$ of affine open subsets in $X$ the corresponding morphism of ind-schemes $\mathcal{L}\left(U^{\prime}\right) \rightarrow \mathcal{L}(U)$ is an open embedding. Further, the ind-scheme $\mathcal{L}(U)_{\text {red }}$ is actually a scheme, namely $\mathcal{L}^{0}(U)$. So our statement follows from the next general fact.

(2.5.3) Lemma. Let $\left(Z_{i}\right)_{i \in I}$ be a finite diagram of ind-schemes in which all the arrows are open embeddings. Assume that the inductive limit of $Z_{i, \text { red }}$ exists in the category of ind-schemes. Then so does the inductive limit of the $Z_{i}$.

Theorem 2.4.2 is proved. Let us also note the following fact.

(2.5.4) Proposition. If $\phi: X \rightarrow Y$ is an étale morphism of schemes of finite type, then $\mathcal{L}(\phi): \mathcal{L}(X) \rightarrow \mathcal{L}(Y)$ is formally étale.

Proof: By the above we can assume that $X=\operatorname{Spec}(A), Y=\operatorname{Spec}(B)$ are affine. If $S$ is a scheme and we have two compatible maps

$$
\alpha: S_{\text {red }} \rightarrow \mathcal{L}(X), \quad \beta: S \rightarrow \mathcal{L}(Y)
$$

we must construct the unique map $S \rightarrow \mathcal{L}(X)$. If $S=\operatorname{Spec} R$, then we have a diagram

$$
\begin{array}{ccc}
A & \stackrel{\alpha^{*}}{\rightarrow} & R_{\mathrm{red}}((t))^{\sqrt{ }}=R_{\mathrm{red}}[[t]] \\
\phi^{*} \uparrow & & \uparrow \\
B & \stackrel{\beta^{*}}{\rightarrow} & R((t))^{\sqrt{ }},
\end{array}
$$

where the right arrow, which is the projection $\rho$ in $(2.3)$, has nilpotent kernel. Therefore there is a unique homomorphism of rings $A \rightarrow R((t))^{\sqrt{ }}$ making the diagram commute. 
(2.6) The formal loop space as an ind-object. For future purposes we construct a certain class of local realizations of $\mathcal{L}(X)$ as an ind-object in the category of schemes.

Let $x_{1}, x_{2}, \ldots, x_{d}$ be the coordinates on the affine space $\mathbb{A}^{d}$. For any $k$-algebra $A$ and any morphism $\phi: \operatorname{Spec} A \rightarrow \mathbb{A}^{d}$ let $\phi^{*}$ be the corresponding map $k\left[x_{1}, \ldots, x_{d}\right] \rightarrow$ A.

(2.6.1) Proposition. Assume that $X=\operatorname{Spec} A$ is an affine scheme and that $\phi: X \rightarrow \mathbb{A}^{d}$ is an étale morphism of schemes.

(a) There is a unique morphism $\theta_{\phi}: \mathcal{L}(X) \rightarrow X$ such that $\theta_{\phi}(f)\left(\phi^{*} x_{i}\right)=$ $f\left(\phi^{*} x_{i}\right)_{0}$ for any $f \in \lambda_{X}(R)$. The restriction of $\theta_{\phi}$ to $\mathcal{L}_{0}(X)$ is equal to the projection $p$ from (2.2.1)(b).

(b) We have $\mathcal{L}\left(\mathbb{A}^{d}\right) \times_{\mathbb{A}^{d}} X \simeq \mathcal{L}(X)$.

Proof: Consider the morphism of functors $\tilde{\lambda}_{\mathbb{A}^{d}} \rightarrow \eta_{\mathbb{A}^{d}}$ which maps a homomorphism $f \in \operatorname{Hom}_{\mathbf{A l g}}\left(k\left[x_{1}, \ldots, x_{d}\right], R((t))\right)$ to the unique morphism in $\operatorname{Hom}_{\mathbf{A l g}}\left(k\left[x_{1}, \ldots, x_{d}\right], R\right)$ such that $x_{i} \mapsto f\left(x_{i}\right)_{0}$. This morphism of functors can be seen as a morphism of ind-schemes $\tilde{\theta}_{\mathbb{A}^{d}}: \tilde{\mathcal{L}}\left(\mathbb{A}^{d}\right) \rightarrow \mathbb{A}^{d}$. It is clear that its restriction to $\mathcal{L}^{0}\left(\mathbb{A}^{d}\right)$ coincides with the projection $p$ from $(2.2 .1)(\mathrm{b})$. Let $\theta_{\mathbb{A}^{d}}$ be the restriction of $\tilde{\theta}_{\mathbb{A}^{d}}$ to the ind-subscheme $\mathcal{L}\left(\mathbb{A}^{d}\right) \subset \tilde{\mathcal{L}}\left(\mathbb{A}^{d}\right)$. Since $\mathcal{L}(X)$ is an inductive limit of nilpotent extensions of the scheme $\mathcal{L}^{0}(X)$ and $\phi$ is formally étale, there is a map $\theta_{\phi}: \mathcal{L}(X) \rightarrow X$ splitting the diagram

$$
\begin{array}{ccc}
\mathcal{L}^{0}(X) & \rightarrow & X \\
\downarrow & & \downarrow \phi \\
\mathcal{L}(X) & \rightarrow & \mathbb{A}^{d}
\end{array}
$$

into two commutative triangles. Here, the lower horizontal arrow is the composition of maps

$$
\theta_{\mathbb{A}^{d}} \circ \mathcal{L}(\phi): \mathcal{L}(X) \rightarrow \mathcal{L}\left(\mathbb{A}^{d}\right) \rightarrow \mathbb{A}^{d}
$$

Let $\psi$ be the resulting map $\mathcal{L}(X) \rightarrow \mathcal{L}\left(\mathbb{A}^{d}\right) \times_{\mathbb{A}^{d}} X$. We claim that $\psi$ is an isomorphism. For this, we construct a map $\chi: \mathcal{L}\left(\mathbb{A}^{d}\right) \times_{\mathbb{A}^{d}} X \rightarrow \mathcal{L}(X)$ inverse to $\psi$. Let $S=\operatorname{Spec} R$ be a scheme. A morphism $S \rightarrow \mathcal{L}\left(\mathbb{A}^{d}\right) \times_{\mathbb{A}^{d}} X$ is a compatible pair

$$
\left(\alpha: S \rightarrow \mathcal{L}\left(\mathbb{A}^{d}\right), \beta: S \rightarrow X\right) .
$$

We need to construct a map $\chi(\alpha, \beta): S \rightarrow \mathcal{L}(X)$. First, $\alpha\left(S_{\text {red }}\right) \subset \mathcal{L}^{0}\left(\mathbb{A}^{d}\right)$, and $\beta\left(S_{\text {red }}\right) \subset X$. Thus, by Proposition 2.2.4, we have a map $\gamma: S_{\text {red }} \rightarrow \mathcal{L}^{0}(X)$. The composition $\tilde{\gamma}$ of $\gamma$ and the embedding $\mathcal{L}^{0}(X) \subset \mathcal{L}(X)$ gives a commutative diagram

$$
\begin{array}{ccc}
S_{\text {red }} & \stackrel{\tilde{\gamma}}{\rightarrow} & \mathcal{L}(X) \\
\downarrow & & \downarrow \mathcal{L}(\phi) \\
S & \stackrel{\alpha}{\rightarrow} & \mathcal{L}\left(\mathbb{A}^{d}\right) .
\end{array}
$$


To complete the construction, we notice that $\mathcal{L}(\phi)$ is formally étale by Proposition 2.5.4 and so we have a map $S \rightarrow \mathcal{L}(X)$ splitting (2.6.2) into two commutative triangles. We take this map to be $\chi(\alpha, \beta)$. The verification that $\chi$ is inverse to $\psi$ is straightforward. Proposition 2.6.1 is proved.

Let $\mathbb{E}$ be the set of $\varepsilon=\left(\varepsilon_{-1}, \varepsilon_{-2}, \ldots\right), \varepsilon_{j} \in \mathbb{Z}_{+}$such that $\varepsilon_{j}=0$ for almost all $j$. It is equipped with the natural partial order: $\varepsilon \leq \varepsilon^{\prime}$ if $\varepsilon_{j} \leq \varepsilon_{j}^{\prime}$ for all $j$. In the remainder of this section we assume that the $k$-scheme $X$ is smooth. Thus $X$ can be covered by affine open sets $U=\operatorname{Spec} A$ possessing étale maps $\phi: U \rightarrow \mathbb{A}^{d}$. For every such $U, \phi$ we consider the functor

$$
\lambda_{\phi}^{\varepsilon}: R \mapsto\left\{f \in \lambda_{U}(R) \mid f\left(\phi^{*} x_{i}\right)_{j}^{1+\varepsilon_{j}}=0, \forall i \in[1, d], \forall j<0\right\} .
$$

(2.6.3) Proposition. The functor $\lambda_{\phi}^{\varepsilon}$ is representable by a closed subscheme $\mathcal{L}^{\varepsilon}(\phi) \subset$ $\mathcal{L}(U)$, such that $\mathcal{L}(U)$ is the inductive limit of the schemes $\mathcal{L}^{\varepsilon}(\phi)$.

Proof: We first consider the case $U=\mathbb{A}^{d}, \phi=$ Id. Let $N$ be such that $\epsilon_{j}=0$ for $j<N$. Define the scheme $\mathcal{L}^{\varepsilon}\left(\mathbb{A}^{d}\right)$ by

$$
\mathcal{L}^{\varepsilon}\left(\mathbb{A}^{d}\right)=\operatorname{Spec}\left(k\left[a_{l}^{(i)} ;-N \leq l\right] /\left(\left(a_{l}^{(i)}\right)^{1+\epsilon_{l}} ; l<0\right)\right)
$$

It is immediate that this scheme represents the functor $\lambda_{\text {Id }}^{\varepsilon}$, and that $\lambda_{\phi}^{\varepsilon}=\lambda_{\text {Id }}^{\varepsilon} \times \lambda_{A^{d}}$ $\lambda_{U}$. The proof of Proposition 2.6.1 implies that that the map

$$
\lambda_{\phi}^{\varepsilon} \rightarrow \lambda_{\mathrm{Id}}^{\varepsilon} \times_{\eta_{\mathbb{A} d}} \eta_{U}, \quad f \mapsto\left(f \circ \phi^{*}, \theta_{\phi}(f)\right),
$$

is an isomorphism of functors. Thus, the closed subscheme

$$
\mathcal{L}^{\varepsilon}\left(\mathbb{A}^{d}\right) \times_{\mathbb{A}^{d}} U \subset \mathcal{L}\left(\mathbb{A}^{d}\right) \times_{\mathbb{A}^{d}} U=\mathcal{L}(U),
$$

see Proposition 2.6.1.(b), represents the functor $\lambda_{\phi}^{\varepsilon}$. It is clear by the definition that $\lambda_{U}=\lim _{\varepsilon} \lambda_{\phi}^{\varepsilon}$ in the category of functors Alg $\rightarrow$ Sets and thus we have that $\mathcal{L}(U)=\lim _{\varepsilon} \overrightarrow{\mathcal{L}^{\varepsilon}}(\phi)$ in the category of ind-schemes.

Let us note the following reformulation of this fact, to be used later.

(2.6.7) Corollary. If $\phi, \psi$ are two étale maps $U \rightarrow \mathbb{A}^{d}$, then the ind-objects "lim $" \mathcal{L}^{\varepsilon}(\phi), ~ " \lim _{\varepsilon} " \mathcal{L}^{\varepsilon}(\psi)$ are isomorphic, i.e., for any $\varepsilon$ there is $\varepsilon^{\prime}$ such that $\mathcal{L}^{\varepsilon}(\phi) \subset \mathcal{L}^{\varepsilon^{\prime}}(\psi)$ and vice versa.

Proof: Given $\psi$, any map $S \rightarrow \mathcal{L}(U)$ with $S$ an affine scheme, factors through some $\mathcal{L}^{\varepsilon^{\prime}}(\psi)$. Now take $S=\mathcal{L}^{\varepsilon}(\phi)$ which we know to be an affine scheme by $(2.6 .4,6)$. 
(2.7) The formal loop space as an ind-pro-object. We keep the notations of (2.6). Thus $U=\operatorname{Spec}(A)$ is affine and $\phi: U \rightarrow \mathbb{A}^{d}$ is étale. The schemes $\mathcal{L}^{\varepsilon}(\phi)$ are of infinite type. In fact, each of these schemes is a projective limit of schemes of finite type, so $\mathcal{L}(U)$ can be viewed as an ind-pro-object in the category of schemes of finite type over $k$. In this subsection we construct explicit ind-pro-systems for $\mathcal{L}(U)$.

Consider the functor $\lambda_{n \phi}^{\varepsilon}: R \mapsto \lambda_{\phi}^{\varepsilon}(R) / \sim_{n \phi}$, where

$$
f \sim_{n \phi} g \Longleftrightarrow f\left(\phi^{*} x_{i}\right)-g\left(\phi^{*} x_{i}\right) \in t^{n+1} R[[t]], \quad \forall i .
$$

(2.7.1) Proposition. (a) The functor $\lambda_{n \phi}^{\varepsilon}$ is representable by a scheme $\mathcal{L}_{n}^{\varepsilon}(\phi)$. The scheme $\mathcal{L}_{n}^{\varepsilon}(\phi)$ is of finite type and is a nilpotent extension of $\mathcal{L}_{n}^{0}(U)=\mathcal{L}_{n}^{0}(\phi)$. Moreover, $\mathcal{L}^{\varepsilon}(\phi)=\lim _{\longleftarrow} \mathcal{L}_{n}^{\varepsilon}(\phi)$.

(b) The schemes $\overleftarrow{\mathcal{L}}_{n}^{\varepsilon}(\phi)$ form an ind-pro-system with Cartesian squares $\left(n^{\prime} \geq\right.$ $\left.n, \varepsilon^{\prime} \geq \varepsilon\right)$

$$
\begin{array}{cccc}
\mathcal{L}_{n^{\prime}}^{\varepsilon}(\phi) & \hookrightarrow & \mathcal{L}_{n^{\prime}}^{\varepsilon^{\prime}}(\phi) \\
\downarrow & & \\
\downarrow & & \\
\mathcal{L}_{n}^{\varepsilon}(\phi) & \hookrightarrow & \mathcal{L}_{n}^{\varepsilon^{\prime}}(\phi)
\end{array}
$$

where the vertical arrows are smooth affine morphisms.

(c) The ind-pro-object " $\stackrel{\lim }{\longrightarrow}_{\varepsilon} " \longleftarrow_{\longleftarrow_{n}} " \mathcal{L}_{n}^{\varepsilon}(\phi)$ is independent, up to isomorphism, on $\phi$.

Proof: Claim $(a)$ is entirely similar to Proposition 2.6.3. We first consider the case $U=\mathbb{A}^{d}, \phi=\operatorname{Id}$ and define the scheme $\mathcal{L}_{n}^{\varepsilon}\left(\mathbb{A}^{d}\right)$ by

$$
\mathcal{L}_{n}^{\varepsilon}\left(\mathbb{A}^{d}\right)=\operatorname{Spec}\left(k\left[a_{l}^{(i)} ;-N \leq l \leq n\right] /\left(\left(a_{l}^{(i)}\right)^{1+\varepsilon_{l}} ; l<0\right)\right), \quad N \gg 0 .
$$

It represents $\lambda_{n \mathrm{Id}}^{\varepsilon}$. The fiber product scheme $\mathcal{L}_{n}^{\varepsilon}\left(\mathbb{A}^{d}\right) \times_{\mathbb{A}^{d}} U$ represents the functor $\lambda_{n \mathrm{Id}}^{\varepsilon} \times \eta_{\eta_{\AA} d} \eta_{U}$. The isomorphism of functors (2.6.5) yields an isomorphism of functors $\lambda_{n \phi}^{\varepsilon} \rightarrow \lambda_{n \mathrm{Id}}^{\varepsilon} \times{ }_{\eta_{\mathbb{A}}} \eta_{U}$. Thus

$$
\mathcal{L}_{n}^{\varepsilon}(\phi)=\mathcal{L}_{n}^{\varepsilon}\left(\mathbb{A}^{d}\right) \times_{\mathbb{A}^{d}} U
$$

Claim (b) is obvious in the case $U=\mathbb{A}^{d}, \phi=\mathrm{Id}$. The general case follows from (2.7.3) since the base change of a smooth affine morphism is still smooth affine, and the base change of a Cartesian square is Cartesian.

Claim (c) follows from Corollary 2.6.7 and Proposition 2.1.2.

Passing to the limit in $\epsilon$ we get the ind-scheme $\mathcal{L}_{n}(\phi)=\underline{\lim }_{\epsilon} \mathcal{L}_{n}^{\epsilon}(\phi)$. It represents the functor $R \mapsto \lambda_{U}(R) / \sim_{n \phi}$. As in (2.7.2) we get

$$
\mathcal{L}_{n}\left(\mathbb{A}^{d}\right)=\lim _{N} \operatorname{Spf} k\left[a_{l}^{(i)}, 0 \leq l \leq n\right]\left[\left[a_{l}^{(i)},-N \leq l<0\right]\right]
$$


and we see that $\mathcal{L}_{n}\left(\mathbb{A}^{d}\right)$ is formally smooth. Further, we see that

$$
\mathcal{L}_{n}(\phi)=\mathcal{L}_{n}\left(\mathbb{A}^{d}\right) \times_{\mathbb{A}^{d}} U
$$

from which we see that $\mathcal{L}_{n}(\phi)$ is also formally smooth. 


\section{The global loop space}

(3.1) Localization on a curve. Consider the Lie algebra Der $k[[t]]$ and the group scheme Aut $k[[t]]$ over $k$. They form a Harish-Chandra pair [BB] [F, §6.1]. By construction, we have the action of this pair on the scheme $\mathcal{L}^{0}(X)$ and on the ind-scheme $\mathcal{L}(X)$ constructed in $\S 2$.

Let $C$ be a smooth curve over $k$. The well known procedure of localization, see loc. cit. and [GKF], gives then a scheme $\mathcal{L}^{0}(X)_{C}$ and an ind-scheme $\mathcal{L}(X)_{C}$ over $C$ defined as follows. Let $C^{\wedge} \rightarrow C$ be the scheme of pairs $\left(c, t_{c}\right)$ where $c \in C$ and $t_{c}$ is a formal coordinate near $c$. Then $C^{\wedge}$ has a natural (Der $k[[t]]$, Aut $\left.k[[t]]\right)$-structure, i.e. a simply transitive Der $k[[t]]$-action extending the fiberwise Aut $k[[t]]$-action. We define

$$
\mathcal{L}^{0}(X)_{C}=C^{\wedge} \times_{\text {Aut } k[[t]]} \mathcal{L}^{0}(X), \quad \mathcal{L}(X)_{C}=C^{\wedge} \times_{\text {Aut } k[[t]]} \mathcal{L}(X) .
$$

If $X$ is affine, we define, in a similar way, the ind-scheme $\tilde{\mathcal{L}}(X)_{C}$ starting from $\tilde{\mathcal{L}}(X)$. Because of the simple transitivity of the Der $k[[t]]$-action on $C^{\wedge}$, the (ind-)schemes thus constructued possess natural connections along $C$ which are compatible with the embeddings $\mathcal{L}^{0}(X)_{C} \subset \mathcal{L}(X)_{C}$ and, for $X$ affine, $\mathcal{L}^{0}(X)_{C} \subset \tilde{\mathcal{L}}(X)_{C}$.

Note that $\mathcal{L}^{0}(X)_{C}$ is nothing but the scheme of infinite jets of morphisms $C \rightarrow X$, so it is easy to describe explicitly the functor represented by $\mathcal{L}^{0}(X)_{C}$ (and also by $\left.\mathcal{L}(X)_{C}, \tilde{\mathcal{L}}(X)_{C}\right)$. Let us introduce some notations. For a scheme $S$ and a morphism $f: S \rightarrow C$ we denote by $\Gamma(f) \subset S \times C$ the graph of $f$. Let $f_{\text {red }}: S_{\text {red }} \rightarrow C$ be the restriction of $f$ to $S_{\text {red }}$. We have then the following sheaves of $k$-algebras on $S \times C$ supported on $\Gamma(f)$ :

- $\mathcal{O}_{f}^{\wedge}$, the completion of $\mathcal{O}_{S \times C}$ along $\Gamma(f)$, i.e. the sheaf of functions on the formal neighborhood of $\Gamma(f)$.

- $\mathcal{K}_{f}^{\wedge}$, the sheaf of functions on the punctured formal neighborhood of $\Gamma(f)$. Thus $\mathcal{K}_{f}^{\wedge}$ is obtained from $\mathcal{O}_{f}^{\wedge}$ by inverting the local equations of the divisor $\Gamma(f) \subset S \times C$.

$-\mathcal{K}_{f}^{\sqrt{ }} \subset \mathcal{K}_{f}^{\wedge}$, the subsheaf of sections whose restriction to $S_{\text {red }} \times C$ lies in $\mathcal{O}_{f_{\text {red }}}^{\wedge} \subset \mathcal{K}_{f_{\text {red }}}^{\wedge}$.

(3.1.2) Proposition. (a) The sheaves $\mathcal{O}_{f}^{\wedge}$ and $\mathcal{K}_{f}^{\sqrt{-}}$ are sheaves of local rings.

(b) The scheme $\mathcal{L}^{0}(X)_{C}$ represents the functor $\lambda_{X, C}^{0}: \mathbf{S c h} \rightarrow$ Sets which associates to a scheme $S$ the set of pairs $(f, \rho)$ where $f: S \rightarrow C$ is a morphism of schemes and $\rho:\left(\Gamma(f), \mathcal{O}_{f}^{\wedge}\right) \rightarrow X$ is a morphism of locally ringed spaces.

(c) The ind-scheme $\mathcal{L}(X)_{C}$ ind-represents the functor $\lambda_{X, C}$ defined as in (b) but with $\mathcal{O}_{f}^{\wedge}$ replaced by $\mathcal{K}_{f}^{\sqrt{ }}$.

(d) Similarly, when $X$ is affine, the ind-scheme $\tilde{\mathcal{L}}(X)_{C}$ ind-represents the functor $\tilde{\lambda}_{X, C}$ defined as in (b) but with $\mathcal{O}_{f}^{\wedge}$ replaced by $\mathcal{K}_{f}^{\wedge}$. 
Proof: (a) By choosing an etale coordinate $y$ on $C$ and using the relative etale coordinate $y-f(s)$ on $C \times S$, we reduce to the case when $C=\mathbb{A}^{1}$ and $f$ is constant with value $0 \in \mathbb{A}^{1}$. Then

$$
\mathcal{O}_{f}^{\wedge}=\mathcal{O}_{S}[[t]], \quad \mathcal{K}_{f}=\mathcal{O}_{S}((t)), \quad \mathcal{K}_{f}^{\sqrt{ }}=\mathcal{O}_{S}((t))^{\sqrt{ }}
$$

and our assertion follows from (2.2.1)(a) and (2.4.4)(b).

(b) The projection $\pi: C^{\wedge} \rightarrow C$ induces, for any scheme $S$, a map of sets

$$
\pi_{S}: \operatorname{Hom}\left(S, \mathcal{L}^{0}(X)_{C}\right) \rightarrow \operatorname{Hom}(S, C)
$$

It is enough to show that for any $f: S \rightarrow C$ the set $\pi_{S}^{-1}(f)$ is naturally identified with the set of $\rho:\left(\Gamma(f), \mathcal{O}_{f}^{\wedge}\right) \rightarrow X$. Further, since both functors $\lambda_{X, C}^{0}$ and $\eta_{\mathcal{L}^{0}(X)_{C}}$ are sheaves of sets on Sch, it is enough to construct such an identification Zariski locally on $S$. But locally on $S$ we have, from the definition (3.1.1):

$$
\pi_{S}^{-1}(f)=\operatorname{Lifts}\left(f, C^{\wedge}\right) \times_{\text {Aut } k[[t]]} \operatorname{Hom}\left(S, \mathcal{L}^{0}(X)\right)
$$

where $\operatorname{Lifts}\left(f, C^{\wedge}\right)$ is the set of $\tilde{f}: S \rightarrow C^{\wedge}$ such that $\pi \tilde{f}=f$. Recall that

$$
\operatorname{Hom}\left(S, \mathcal{L}^{0}(X)\right)=\operatorname{Hom}_{\mathbf{L r s}}\left(\left(S, \mathcal{O}_{S}[[t]]\right), X\right)
$$

This means that

$$
\pi_{S}^{-1}(f)=\operatorname{Hom}_{\mathbf{L r s}}((S, \mathcal{A}), X)
$$

where $\mathcal{A}$ is the sheaf of rings on $S$ associated to the presheaf

$$
U \mapsto\left(\prod_{\tilde{f} \in \operatorname{Lifts}\left(\left.f\right|_{U}, C^{\wedge}\right)} \mathcal{O}_{U}[[t]]\right)^{\operatorname{Aut} k[[t]]} .
$$

But it is clear that $\mathcal{A} \simeq \mathcal{O}_{f}^{\wedge}$, whence the statement. Parts (c) and (d) are proved similarly.

(3.2) Factorization monoids. Let $C$ be a smooth curve, as before. For any surjection $J \rightarrow I$ of finite sets and $i \in I$ we denote by $J_{i}$ the preimage of $i$. To such a surjection one associates, in a standard way, the "diagonal" embedding $\Delta^{(J / I)}$ : $C^{I} \hookrightarrow C^{J}$. Let $U^{(J / I)} \subset C^{J}$ be the locus of $\left(c_{j}\right)_{j \in J}$ such that $c_{j} \neq c_{j^{\prime}}$ whenever the images of $j$ and $j^{\prime}$ in $I$ are different. We denote by $j^{(J / I)}: U^{(J / I)} \hookrightarrow C^{J}$ the embedding. 
(3.2.1) Definition. Let $Y$ be an ind-scheme over $C$. A factorization monoid structure on $Y$ is a collection of ind-schemes $Y_{I}$ over $C^{I}$ with a formally integrable connection, given for each nonempty finite set $I$ such that $Y_{\{1\}}=Y$ and $Y_{I}$ is formally smooth over $C^{I}$, equipped with the following data:

(a) An isomorphism of $C^{I}$-ind-schemes $\nu^{(J / I)}: \Delta^{(J / I) *} Y_{J} \stackrel{\sim}{\rightarrow} Y_{I}$ for every surjection $J \rightarrow I$, compatible with compositions of surjections.

(b) An isomorphism of $U^{(J / I)}$-ind-schemes

$$
\kappa^{(J / I)}: j^{(J / I) *}\left(\prod_{i \in I} Y_{J_{i}}\right) \stackrel{\sim}{\rightarrow} j^{(J / I) *} Y_{J}
$$

for every $J \rightarrow I$, such that for $K \rightarrow J$ the isomorphism $\kappa^{(K / I)}$ coincides with the composition $\kappa^{(J / I)} \circ\left(\prod_{i \in I} \kappa^{\left(K_{i} / J_{i}\right)}\right)$. We also demand that $\nu, \kappa$ are compatible in the following sense : for every $J \rightarrow J^{\prime} \rightarrow I$ one has $\nu^{\left(J / J^{\prime}\right)} \circ \Delta^{\left(J / J^{\prime}\right) *}\left(\kappa^{(J / I)}\right)=$ $\kappa^{\left(J^{\prime} / I\right)} \circ\left(\bigotimes_{i \in I} \nu^{\left(J_{i} / J_{i}^{\prime}\right)}\right)$.

(3.2.2) Remarks. (a) This is a nonlinear counterpart of the concept of a factorization algebra due to Beilinson and Drinfeld [BD1] [G], see also (6.1) below. factorization monoids can be used to construct factorization algebras by applying "natural" linear constructions.

(b) More generally, we can speak about a factorization monoid structure on any functor $\mathcal{Y}: \mathbf{S c h} \rightarrow$ Sets (not necessarily representable by an ind-scheme) which is equipped with a morphism to $C$ (i.e. to the representable functor $\eta_{C}$ ).

(3.2.3) Example. Let $G$ be an affine algebraic group over $k$. Then $\mathcal{L}^{0}(G)$ is a group scheme and $\tilde{\mathcal{L}}(G)$ is a group ind-scheme over $C$. The quotient ind-scheme $\mathfrak{G} r_{G}=\tilde{\mathcal{L}}(G) / \mathcal{L}^{0}(G)$ is known as the affine Grassmannian associated to $G$. The natural family of such Grassmannians over $C$, i.e. the ind-scheme $\mathfrak{G} r_{G}(C)=$ $\tilde{\mathcal{L}}(G)_{C} / \mathcal{L}^{0}(G)_{C}$ is known to have a structure of a factorization monoid, see $[\mathrm{G}$, $\S 5.2 .2]$.

Now, the main result of this section is the following.

(3.2.4) Theorem. Let $X$ be a scheme of finite type. Then the $C$-scheme $\mathcal{L}^{0}(X)_{C}$ and the $C$-ind-scheme $\mathcal{L}(X)_{C}$ possess natural structures of factorization monoids so that the embedding $\mathcal{L}^{0}(X)_{C} \subset \mathcal{L}(X)_{C}$ is a factorization homomorphism. Similarly, if $X$ is affine, then the $C$-ind-scheme $\tilde{\mathcal{L}}(X)_{C}$ has a natural structure of a factorization monoid so that the embedding $\mathcal{L}^{0}(X)_{C} \subset \tilde{\mathcal{L}}(X)_{C}$ is a factorization homomorphism.

(3.3) Factorization monoid structure on the functors represented by $\mathcal{L}^{0}(X)_{C}, \tilde{\mathcal{L}}(X)_{C}$ and $\mathcal{L}(X)_{C}$. Let $S$ be a scheme and $f_{I}$ be $I$-uple of morphisms $f_{i}: S \rightarrow C, i \in I$. We denote by $\Gamma\left(f_{I}\right) \subset S \times C$ the union of the graphs of the $f_{i}$ 's and by $f_{I \text {,red }} \subset S_{\text {red }} \times C$ the union of the graphs of the $f_{i \text {,red }}$. Let us introduce, similarly to (3.1), the following sheaves of rings on $S \times C$ with support in $\Gamma\left(f_{I}\right)$ : 
- $\mathcal{O}_{f_{I}}^{\wedge}$, the completion of $\mathcal{O}_{S \times C}$ along $\Gamma\left(f_{I}\right)$, i.e. the sheaf of functions on the formal neighborhood of $\Gamma\left(f_{I}\right)$.

- $\mathcal{K}_{f_{I}}^{\wedge}$, the sheaf of functions on the punctured formal neighborhood of $\Gamma\left(f_{I}\right)$.

$-\mathcal{K}_{f_{I}}^{\sqrt{ }} \subset \mathcal{K}_{f_{I}}^{\wedge}$, the subsheaf of sections whose restriction to $S_{\text {red }} \times C$ lies in $\mathcal{O}_{f_{I, \text { red }}}^{\wedge} \subset \mathcal{K}_{f_{I, \text { red }}}^{\wedge}$

(3.3.1) Proposition. The sheaves $\mathcal{O}_{f_{I}}^{\wedge}$ and $\mathcal{K}_{f_{I}}^{\sqrt{ }}$ are sheaves of local rings.

Proof: The case of $\mathcal{O}_{f_{I}}^{\wedge}$ is immediate because $\left(\Gamma\left(f_{I}\right), \mathcal{O}_{f_{I}}^{\wedge}\right)$ is the formal neighborhood of $\Gamma\left(f_{I}\right)$ in $S \times C$, hence it is a locally ringed space. The case of $\mathcal{K}_{f_{I}}^{\sqrt{ }}$ is analog to the proof of Proposition 2.2.1. Indeed we can assume that $C=\operatorname{Spec} k[t]$ and $S=\operatorname{Spec} R$. Set $b_{i}=f_{i}^{*}(t), K_{f_{I}}^{\sqrt{ }}=H^{0}\left(\Gamma\left(f_{I}\right), \mathcal{K}_{f_{I}}^{\sqrt{ }}\right)$, and $O_{f_{I}}^{\wedge}=H^{0}\left(\Gamma\left(f_{I}\right), \mathcal{O}_{f_{I}}^{\wedge}\right)$. The ring $K_{f_{I}}^{\sqrt{ }}$ is identified with the series $\sum_{l>>-\infty} a_{l}(t) \prod_{i}\left(t-b_{i}\right)^{l}$ where $a_{l}(t) \in R[t]$ are polynomials of degree less than $|I|$ with nilpotent coefficients if $l<0$, and the subring $O_{f_{I}}^{\wedge}$ with the series such that $a_{l}(t)=0$ if $l<0$, see Example (3.7). We have $\underline{\operatorname{Spec}}\left(K_{f_{I}}^{\sqrt{ }}\right)=\underline{\operatorname{Spec}}\left(O_{f_{I}}^{\wedge}\right)$ because $K_{f_{I}}^{\sqrt{ }}$ is a nilpotent extension of $O_{f_{I}}^{\wedge}$. Let $i: \Gamma \overline{\left(f_{I}\right)} \rightarrow \underline{\operatorname{Spec}}\left(O_{f_{I}}^{\widehat{\Lambda}}\right)$ be the natural map. Then

$$
i^{-1} \mathcal{O}_{\operatorname{Spec}\left(K_{f_{I}}^{-}\right)}=\mathcal{K}_{f_{I}}^{\sqrt{ }}
$$

The proposition follows.

(3.3.2) Definition. Let I be a nonempty finite set.

(a) We define the contravariant functors $\lambda_{X, C^{I}}^{0}, \lambda_{X, C^{I}}$ from Sch to Sets as follows. For a scheme $S$ the set $\lambda_{X, C^{I}}^{0}(S)$ consists of pairs $\left(f_{I}, \rho\right)$ such that

$$
f_{I} \in \operatorname{Hom}_{\mathbf{S c h}_{k}}\left(S, C^{I}\right) \quad \text { and } \quad \rho \in \operatorname{Hom}_{\mathbf{L r s}_{k}}\left(\left(\Gamma\left(f_{I}\right), \mathcal{O}_{f_{I}}^{\wedge}\right), X\right)
$$

The contravariant functor $\lambda_{X, C^{I}}$ is defined similarly but with $\mathcal{O}_{f_{I}}^{\wedge}$ replaced by $\mathcal{K}_{f_{I}}^{\sqrt{ }}$.

(b) If $X$ is affine, the functor $\tilde{\lambda}_{X, C^{I}}$ from Alg to Sets is such that the set $\tilde{\lambda}_{X, C^{I}}(R)$ consists of pairs $\left(f_{I}, \rho\right)$ with

$$
f_{I} \in \operatorname{Hom}_{\mathbf{S c h}_{k}}\left(\operatorname{Spec} R, C^{I}\right) \quad \text { and } \quad \rho \in \operatorname{Hom}_{\mathbf{A l g}_{k}}\left(k[X], \mathrm{H}^{0}\left(\Gamma\left(f_{I}\right), \mathcal{K}_{f_{I}}^{\wedge}\right)\right) .
$$

The embeddings of sheaves of rings $\mathcal{O}_{f_{I}}^{\wedge} \hookrightarrow \mathcal{K}_{f_{I}}^{\sqrt{ }} \hookrightarrow \mathcal{K}_{f_{I}}^{\wedge}$ induce embeddings of functors $\lambda_{X, C^{I}}^{0} \hookrightarrow \lambda_{X, C^{I}} \hookrightarrow \tilde{\lambda}_{X, C^{I}}$. 
(3.3.3) Proposition. Let $X$ be a fixed scheme of finite type. The families of functors $\left(\lambda_{X, C^{I}}^{0}\right),\left(\tilde{\lambda}_{X, C^{I}}\right),\left(\lambda_{X, C^{I}}\right)$ with I running over nonempty finite sets, form each a factorization monoid in the category of functors.

Proof: This is almost obvious by construction. Indeed, let $J \rightarrow I$ be a surjection of nonempty sets. Then $\Delta^{(J / I) *}$ of the $J$ th functor in any of the three families takes $S$ into the set of $\left(f_{J}, \rho\right)$ where $f_{J}$ is a morphism $S \rightarrow C^{J}$ which in fact lie in the image of $\Delta^{(J / I)}$. Thus $f_{J}$ comes from a uniquely defined $I$-tuple $f_{I}$. Now, $\Gamma\left(f_{J}\right)=\Gamma\left(f_{I}\right)$ and so each of the three sheaves $\mathcal{O}^{\wedge}, \mathcal{K}^{\wedge}, \mathcal{K}^{\sqrt{ }}$ associated to them coincide. This gives the datum (a) of Definition 3.2.1. Similarly, $j^{(J / I)^{*}}$ applied to the $J$ th functor in any of the families, takes $S$ into the set of $\left(f_{J}, \rho\right)$ where $f_{J}: S \rightarrow U^{(J / I)}$. But this means that $\Gamma\left(f_{J}\right)=\coprod_{i \in I} \Gamma\left(f_{J_{i}}\right)$ and hence on the level of set of morphisms of $\Gamma\left(f_{J}\right)$ equipped with any of the three sheaves of rings, we get a direct product. This gives the datum (b), i.e. the isomorphism $\kappa^{(J / I)}$. The associativity of these isomorphisms is obvious.

Therefore, to establish Theorem 3.2.4, we need only to prove the representability of the functors $\lambda_{X, C^{I}}^{0}, \lambda_{X, C^{I}}$ and, when $X$ is affine, of $\tilde{\lambda}_{X, C^{I}}$, as well as to prove the formal smoothness of the structure morphisms of the representing objects to $C^{I}$.

\section{(3.4) The global space of germs of arcs.}

(3.4.1) Proposition. The functor $\lambda_{X, C^{I}}^{0}$ is representable by a scheme $\mathcal{L}^{0}(X)_{C^{I}}$ of infinite type over $C^{I}$.

Proof: For $n>0$ let $C^{(n)}$ be the $n$th symmetric product of $C$. As $C$ is a smooth curve, $C^{(n)}$ is identified with the Hilbert scheme $\operatorname{Hilb}^{n}(C)$ parametrizing subschemes in $C$ of finite length $n$. Explicitly, to a point of $C^{(n)}$, i.e. an effective divisor $D$ on $C$ of degree $n$, there corresponds the subscheme $Z_{D}=\operatorname{Spec}\left(\mathcal{O}_{C} / \mathcal{O}_{C}(-D)\right)$. The following lemma is well-known.

(3.4.2) Lemma. Let $T$ be a $k$-scheme, and $X, Z$ be any $T$-schemes. Assume that the morphism $Z \rightarrow T$ is finite, and that $X \rightarrow T$ is of finite type. The contravariant functor

$$
\text { Sch } \rightarrow \text { Sets }, \quad S \mapsto \operatorname{Hom}_{T}(S \times Z, X),
$$

is represented by a T-scheme $\underline{\mathrm{Hom}}_{T}(Z, X)$.

Let $Z \subset C \times C^{(n)}$ be the total space of the family of the schemes $Z_{D}, D \in C^{(n)}$. We set $\operatorname{Map}^{n}(C, X)=\underline{\operatorname{Hom}}_{C^{(n)}}\left(Z, X \times C^{(n)}\right)$. Let $u_{I}^{n}$ be the composition of the maps

$$
C^{I} \rightarrow C^{I \times[1, n+1]} \rightarrow C^{((n+1)|I|)}
$$


where the first map takes the $I$-uple $\left(c_{i}, i \in I\right)$ to the $I \times[1, n+1]$-uple $\left(c_{i}, c_{i}, \ldots, c_{i}, i \in\right.$ $I$ ), each $c_{i}$ counted $n+1$ times, and the second map is the projection from the Cartesian product to the symmetric product. Let $\mathcal{L}_{n}^{0}(X)_{C^{I}}$ be the fiber product

$$
\mathcal{L}_{n}^{0}(X)_{C^{I}}=C^{I} \times_{C((n+1)|I|)} \operatorname{Map}^{(n+1)|I|}(C, X) .
$$

For any $I$-uple $c \in C^{I}$ we have inclusions of subschemes of $C$

$$
u_{I}^{0}(c) \subset u_{I}^{1}(c) \subset u_{I}^{2}(c) \subset \cdots
$$

Thus we get a projective system of schemes

$$
\mathcal{L}_{1}^{0}(X)_{C^{I}} \leftarrow \mathcal{L}_{2}^{0}(X)_{C^{I}} \leftarrow \mathcal{L}_{3}^{0}(X)_{C^{I}} \leftarrow \cdots
$$

The morphisms in this projective system are affine because the embeddings $u_{I}^{n}(c) \subset$ $u_{I}^{n+1}(c)$ are purely nilpotent. Therefore we have the limit scheme

$$
\mathcal{L}^{0}(X)_{C^{I}}=\lim _{\leftarrow_{n}} \mathcal{L}_{n}^{0}(X)_{C^{I}}
$$

We claim that the scheme $\mathcal{L}^{0}(X)_{C^{I}}$ represents the functor $\lambda_{X, C^{I}}^{0}$. Indeed a morphism from a $k$-scheme $S$ to $\mathcal{L}_{n}^{0}(X)_{C^{I}}$ is a pair $\left(f_{I}, \rho\right)$ where $f_{I}: S \rightarrow C^{I}$ and $\rho: u_{I}^{n}\left(f_{I}\right) \rightarrow X$ are morphisms of schemes. Here $u_{I}^{n}\left(f_{I}\right) \subset S \times C$ is the subscheme of relative length $n|I|$ over $S$ corresponding to the $S$-point $f_{I}$ of $C^{I}$ via $u_{I}^{n}$. When we pass to the limit we get $\lim _{n} \mathcal{O}_{u_{I}^{n}\left(f_{I}\right)}=\mathcal{O}_{f_{I}}^{\wedge}$.

(3.4.5) Remark. When $X=\operatorname{Spec} A$ is affine, the scheme $\mathcal{L}^{0}(X)_{C}$, being the scheme of infinite jets of maps $C \rightarrow X$, is the spectrum of a commutative $\mathcal{O}_{C^{-}}$ algebra with connection along $C$ (in fact, of the universal such algebra with connection generated by $\mathcal{O}_{C} \otimes_{k} A$ ). According to Beilinson and Drinfeld [BD1], [G], commutative $\mathcal{O}_{C}$-algebras with connection are particular case of chiral algebras which, in their turn, give factorization algebras. So our construction in this case is a particular case of theirs.

(3.4.6) Proposition. Let $\phi: X \rightarrow Y$ be an étale morphism of schemes of finite type, and $\pi: C \rightarrow D$ be a morphism of smooth curves. Then

(a) if $\pi$ is étale then each morphism $\mathcal{L}_{n}^{0}(\phi)_{\pi^{I}}: \mathcal{L}_{n}^{0}(X)_{C^{I}} \rightarrow \mathcal{L}_{n}^{0}(Y)_{D^{I}}$ is étale,

(b) the square

$$
\begin{array}{cccc}
\mathcal{L}_{n}^{0}(X)_{C^{I}} & \rightarrow & \mathcal{L}_{n}^{0}(Y)_{D^{I}} \\
\downarrow & & \downarrow \\
\mathcal{L}_{0}^{0}(X)_{C^{I}} & \rightarrow & \mathcal{L}_{0}^{0}(Y)_{D^{I}},
\end{array}
$$

as well as the analogous square for $\mathcal{L}^{0}(X)_{D^{I}}, \mathcal{L}^{0}(Y)_{D^{I}}$, is Cartesian. 
Proof: (a) Let $S$ be any scheme. Given two compatible morphisms

$$
\alpha=\left(\alpha_{I}, \rho_{\alpha}\right): S_{\mathrm{red}} \rightarrow \mathcal{L}_{n}^{0}(X)_{C^{I}}, \quad \beta=\left(\beta_{I}, \rho_{\beta}\right): S \rightarrow \mathcal{L}_{n}^{0}(Y)_{D^{I}},
$$

we must prove that there is a unique morphism $\gamma=\left(\gamma_{I}, \rho_{\gamma}\right): S \rightarrow \mathcal{L}_{n}^{0}(X)_{C^{I}}$ which splits the square

$$
\begin{array}{ccc}
S_{\text {red }} & \rightarrow & \mathcal{L}_{n}^{0}(X)_{C^{I}} \\
\downarrow & & \downarrow \\
S & \rightarrow & \mathcal{L}_{n}^{0}(Y)_{D^{I}},
\end{array}
$$

into two commutative triangles. We have a commutative square

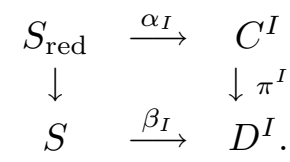

Thus, $\pi$ being étale, there is a unique morphism $\gamma_{I}: S \rightarrow C^{I}$ splitting (3.4.7) into two commutative triangles. Let the subschemes

$$
u_{I}^{n}\left(\alpha_{I}\right) \subset S_{\text {red }} \times C, \quad u_{I}^{n}\left(\beta_{I}\right) \subset S \times D, \quad u_{I}^{n}\left(\gamma_{I}\right) \subset S \times C
$$

be as in (3.4). We have a Cartesian square

$$
\begin{array}{ccc}
u_{I}^{n}\left(\alpha_{I}\right) & \rightarrow & S_{\text {red }} \times C \\
\downarrow & & \downarrow \\
u_{I}^{n}\left(\gamma_{I}\right) & \rightarrow & S \times C,
\end{array}
$$

yielding a nilpotent extension of schemes $u_{I}^{n}\left(\alpha_{I}\right) \rightarrow u_{I}^{n}\left(\gamma_{I}\right)$. This map fits into a commutative diagram

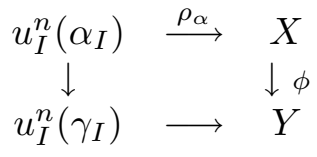

where the lower arrow is the composition of the chain of maps

$$
u_{I}^{n}\left(\gamma_{I}\right) \stackrel{\operatorname{Id} \times \pi}{\longrightarrow} u_{I}^{n}\left(\beta_{I}\right) \stackrel{\rho_{\beta}}{\longrightarrow} Y .
$$

Thus, $\phi$ being étale, there is a unique morphism $\rho_{\gamma}: u_{I}^{n}\left(\gamma_{I}\right) \rightarrow X$ splitting (3.4.8) into two commutative triangles. We have proved (a).

(b) Let

$$
\psi: \mathcal{L}_{n}^{0}(X)_{C^{I}} \rightarrow \mathcal{L}_{0}^{0}(X)_{C^{I}} \times_{\mathcal{L}_{0}^{0}(Y)_{D^{I}}} \mathcal{L}_{n}^{0}(Y)_{D^{I}}
$$


be the morphism induced by the diagram. To prove that $\psi$ is an isomorphism we show that for every scheme $S$ and every two compatible morphisms

$$
\alpha: S \rightarrow \mathcal{L}_{0}^{0}(X)_{C^{I}}, \quad \beta: S \rightarrow \mathcal{L}_{n}^{0}(Y)_{D^{I}}
$$

there is a unique $\gamma: S \rightarrow \mathcal{L}_{n}^{0}(X)_{C^{I}}$ such that $\psi(\gamma)=(\beta, \alpha)$. By definition $\alpha=\left(\alpha_{I}, \rho_{\alpha}\right)$ with $\alpha_{I}: S \rightarrow D^{I}, \rho_{\alpha}: u_{I}^{n}\left(\alpha_{I}\right) \rightarrow Y$ morphisms of schemes. Similarly $\beta=\left(\beta_{I}, \rho_{\beta}\right)$ with $\beta_{I}: S \rightarrow C^{I}, \rho_{\beta}: \Gamma\left(\beta_{I}\right) \rightarrow X$. We look for $\gamma=\left(\gamma_{I}, \rho_{\gamma}\right)$ with $\gamma_{I}: S \rightarrow C^{I}, \rho_{\gamma}: u_{I}^{n}\left(\gamma_{I}\right) \rightarrow X$.

We first prove the existence of $\gamma$. Take $\gamma_{I}=\beta_{I}$. Next, by compatibility of $\alpha$ and $\beta$ we have $(\operatorname{Id} \times \pi)\left(\Gamma\left(\beta_{I}\right)\right)=\Gamma\left(\alpha_{I}\right)$, and this implies that $\operatorname{Id} \times \pi$ induces a morphism $\varpi: u_{I}^{n}\left(\beta_{I}\right) \rightarrow u_{I}^{n}\left(\alpha_{I}\right)$. Composing $\varpi$ with $\rho_{\alpha}$ we get a diagram

$$
\begin{array}{ccc}
\Gamma\left(\beta_{I}\right) & \rightarrow & X \\
\downarrow & & \downarrow \\
u_{I}^{n}\left(\beta_{I}\right) & \rightarrow & Y,
\end{array}
$$

with horizontal arrows $\rho_{\beta}, \rho_{\alpha} \circ \varpi$ and right vertical arrow $\pi$. This diagram is commutative by compatibility of $\alpha$ and $\beta$. Now the left vertical arrow is a nilpotent embedding, while the right vertical arrow is étale. Therefore there is a unique morphism $\rho_{\gamma}: u_{I}^{n}\left(\beta_{I}\right) \rightarrow X$ splitting the diagram intotwo commutative triangles.

We now check the unicity of $\gamma$. That $\psi(\gamma)=(\beta, \alpha)$ means that, first, $\beta_{I}=\gamma_{I}$ and the diagram (whose horizontal maps are $\rho_{\beta}, \rho_{\gamma}$ respectively)

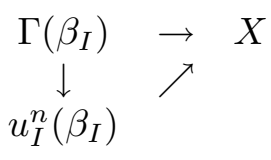

commutes and, second, $\alpha_{I}=\pi^{I} \circ \gamma_{I}$ and the diagram (whose horizontal maps are $\phi \circ \rho_{\gamma}, \rho_{\alpha}$ respectively)

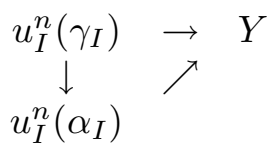

commutes. The second condition determines $\phi \circ \rho_{\gamma}$ uniquely as $\rho_{\alpha} \circ \varpi$. So $\rho_{\gamma}$ splits the following square into two commutative triangles

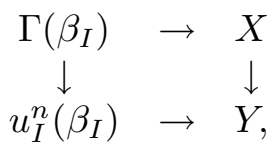

so it is unique because $\phi$ is étale.

(3.5) The meromorphic loop space of an affine scheme. Here we prove the ind-representability of the functor $\tilde{\lambda}_{X, C^{I}}$ for $X$ affine. We first treat the case when 
$X=\mathbb{A}^{1}$. Let $I$ be fixed. To every point $\left(c_{i}\right)_{i \in I} \in C^{I}$ we associate the effective divisor $\sum c_{i}$ on $C$. For every $m, n \geq 0$ let $\mathcal{A}_{m n}$ be the vector bundle on $C^{I}$ whose fiber over $\left(c_{i}\right)$ is the vector space of global sections of the coherent 0-dimensional sheaf

$$
\mathcal{O}_{C}\left(m \sum c_{i}\right) / \mathcal{O}_{C}\left(-n \sum c_{i}\right)
$$

on $C$. Thus $\operatorname{rk}\left(\mathcal{A}_{m n}\right)=|I|(m+n)$. Let $A_{m n}$ be the total space of the bundle $\mathcal{A}_{m n}$, considered as an algebraic variety over $C^{I}$. When $m, n$ vary, these varieties form a double inductive-projective system and the following is then obvious.

(3.5.1) Proposition. (a) For every $m>0$ the limit $\underset{n}{\lim } A_{m n}$ exists as a scheme. The ind-scheme $\tilde{\mathcal{L}}\left(\mathbb{A}^{1}\right)_{C^{I}}=\lim _{m} \lim _{n} A_{m n}$ represents the functor $\tilde{\lambda}_{\mathbb{A}^{1}, C^{I}}$.

(b) $\tilde{\mathcal{L}}\left(\mathbb{A}^{1}\right)_{C^{I}}$ has a natural structure of a $k$-algebra object in the category of indschemes over $C^{I}$.

Proof: A morphism of $k$-schemes $\operatorname{Spec} R \rightarrow A_{m n}$ is a pair of a morphism of $k$ schemes $f_{I}: S \rightarrow C^{I}$, and a morphism of sheaves of $k$-algebras $\mathcal{O}_{A_{m n}} \otimes_{\mathcal{O}_{C I}} R \rightarrow R$ (here, the right hand side is identified with the constant sheaf on $C^{I}$ ). There is a canonical isomorphism

$\lim _{m} \lim _{n} \operatorname{Hom}\left(\mathcal{O}_{A_{m n}} \otimes_{\mathcal{O}_{C} I} R, R\right) \simeq \mathrm{H}^{0}\left(\Gamma\left(f_{I}\right), \mathcal{K}_{f_{I}}\right) \simeq \operatorname{Hom}_{\mathbf{A l g}}\left(k[x], \mathrm{H}^{0}\left(\Gamma\left(f_{I}\right), \mathcal{K}_{f_{I}}\right)\right)$. Claim $(a)$ is proved. In part $(b)$, the variety $A_{m n}$ is not a ring scheme. However there is an obvious map $A_{m n} \times A_{m^{\prime} n^{\prime}} \rightarrow A_{m+m^{\prime}, n+n^{\prime}}$ which induces a ring structure on the limit of the ind-pro system.

(3.5.2) Proposition. For any affine $X$ of finite type the functor $\tilde{\lambda}_{X, C^{I}}$ is representable by an ind-scheme $\tilde{\mathcal{L}}(X)_{C^{I}}$ over $C^{I}$.

Proof: By part (b) of Proposition 3.5.1, for every $f \in k\left[x_{1}, \ldots, x_{d}\right]$ we have a morphism of ind-schemes

$$
\tilde{\mathcal{L}}(f)_{C^{I}}:\left(\tilde{\mathcal{L}}\left(\mathbb{A}^{1}\right)_{C^{I}}\right)^{d} \rightarrow \tilde{\mathcal{L}}\left(\mathbb{A}^{1}\right)_{C^{I}}
$$

If now the scheme $X$ is given in some $\mathbb{A}^{d}$ be equations $f_{j}\left(x_{1}, \ldots, x_{d}\right)=0$, then $\tilde{\lambda}_{X, C^{I}}$ is represented by the ind-scheme $\tilde{\mathcal{L}}(X)_{C^{I}}$ which is the intersection, in $\left(\tilde{\mathcal{L}}\left(\mathbb{A}^{1}\right)_{C^{I}}\right)^{d}$, of the preimages of 0 under the $\tilde{\mathcal{L}}\left(f_{j}\right)_{C^{I}}$, i.e. the inverse limit of an obvious diagram in the category of ind-schemes.

(3.5.3) Corollary. For an affine scheme $X$ the functor $\lambda_{X, C^{I}}$ is represented by an ind-scheme $\mathcal{L}(X)_{C^{I}}$ which is the inductive limit of the formal neighborhoods of $\mathcal{L}^{0}(X)_{C^{I}}$ in the schemes of an inductive system for $\tilde{\mathcal{L}}(X)_{C^{I}}$.

(3.6) The global loop space of an arbitrary scheme. Let now $X$ be an arbitrary scheme of finite type. The (ind-)representability of the functor $\lambda_{X, C^{I}}$ follows from Corollary 3.5.3 for the affine case and from the general gluing properties of the functors summarized in the next proposition. 
(3.6.1) Proposition. (a) The functor $\lambda_{X, C^{I}}$ is a sheaf on $\mathbf{S c h}$.

(b) If $U \subset X$ is an open subset, then the induced morphism of functors $\lambda_{U, C^{I}} \rightarrow$ $\lambda_{X, C^{I}}$ is open.

(c) Let $\left\{U_{\alpha}\right\}_{\alpha \in A}$ be an open covering of $X$. Then $\lambda_{X, C^{I}}$ is equal to the cokernel, in the category $\mathbf{S h f}$, of the pair of morphisms

$$
\prod_{\alpha, \beta} \lambda_{U_{\alpha} \cap U_{\beta}, C^{I}} \rightrightarrows \prod_{\alpha} \lambda_{U_{\alpha}, C^{I}}
$$

Proof: (a) For any $f_{I}: S \rightarrow C^{I}$ the graph $\Gamma\left(f_{I}\right)$ is identified with $S$, so $\mathcal{K}_{f_{I}}^{\sqrt{ }}$ can be regarded as a sheaf of local rings on $S$. So our statement follows from the fact that the representable functor $\eta_{X}$ on Lrs is a sheaf.

(b) Let $S$ be a scheme and $u$ a morphism of functors $\eta_{S} \rightarrow \lambda_{X, C^{I}}$. WE need to prove that the fiber product of $\eta_{S}$ and $\lambda_{U, C^{I}}$ over $\lambda_{X, C^{I}}$ is represented by a scheme $S^{\prime}$ whose natural morphism to $S$ is an open embedding. To see this, we view $u$ as an element of $\lambda_{X, C^{I}}(S)$, so $u=\left(f_{I}, \rho\right)$ with $f_{I}: S \rightarrow C^{I}$ and $\rho:\left(\Gamma\left(f_{I}\right), \mathcal{K}_{f_{I}}^{\sqrt{ }}\right) \rightarrow X$. Notice that $\Gamma\left(f_{I}\right) \simeq S$, so $\rho$ gives, in particular, a continuous map of topological spaces $\bar{\rho}: S \rightarrow X$. It is clear then that the fiber product mentioned above is represented by the open subset $S^{\prime}=\bar{\rho}^{-1}(U) \subset S$.

(c) This follows from (b) and from Lemma 2.4.7(c).

An immediate corollary of Proposition 3.6.1 and Corollary 3.5.3 is that the functor $\lambda_{X, C^{I}}$ is representable by an ind-scheme $\mathcal{L}(X)_{C^{I}}$ over $C^{I}$. Then, Proposition 3.3.3 implies that the collections of schemes and ind-schemes $\mathcal{L}^{0}(X)_{C^{I}}$ and $\mathcal{L}(X)_{C^{I}}$ are factorization monoids in the categories of schemes and ind-schemes. To finish the proof of Theorem 3.2.4 it remains to establish part (b) of the following.

(3.6.2) Proposition. (a) If $\phi: X \rightarrow Y, \pi: C \rightarrow D$ are étale morphisms, then the induced morphism $\mathcal{L}(\phi)_{\pi^{I}}: \mathcal{L}(X)_{C^{I}} \rightarrow \mathcal{L}(Y)_{D^{I}}$ is formally étale.

(b) If $X$ is smooth then the morphism $\mathcal{L}(X)_{C^{I}} \rightarrow C^{I}$ is formally smooth.

Proof: To prove Claim (b) it is sufficient to observe that if $U \subset X, V \subset C$ are affine open sets with étale maps $U \rightarrow \mathbb{A}^{d}, V \rightarrow \mathbb{A}^{1}$ then the composition of maps

$$
\mathcal{L}(U)_{V^{I}} \rightarrow \mathcal{L}\left(\mathbb{A}^{d}\right)_{\mathbb{A}^{I}} \rightarrow \mathbb{A}^{I}
$$

is formally smooth by Claim $(a)$ and the statement (3.7.5) of the example below.

The proof of Claim $(a)$ is similar to that of Proposition 2.5.4. Let $S$ be a scheme. We are given $\alpha: S_{\text {red }} \rightarrow \mathcal{L}(X)_{C^{I}}, \beta: S \rightarrow \mathcal{L}(Y)_{D^{I}}$, and we look for a unique $\gamma$ which splits the square

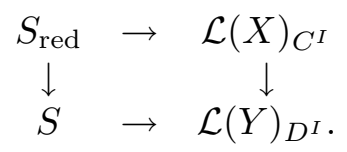


The morphism $\alpha$ consists of a pair $\left(\alpha_{I}: S_{\text {red }} \rightarrow C^{I}, \rho_{\alpha}:\left(\Gamma\left(\alpha_{I}\right), \mathcal{K}_{\alpha_{I}}^{\sqrt{ }}\right) \rightarrow X\right)$. Similarly $\beta$ consists of a pair $\left(\beta_{I}: S \rightarrow D^{I}, \rho_{\beta}:\left(\Gamma\left(\beta_{I}\right), \mathcal{K}_{\beta_{I}}^{\sqrt{ }}\right) \rightarrow Y\right)$. We must construct a pair $\left(\gamma_{I}: S \rightarrow C^{I}, \rho_{\gamma}:\left(\Gamma\left(\gamma_{I}\right), \mathcal{K}_{\gamma_{I}}^{\sqrt{ }}\right) \rightarrow X\right)$. There is a map $\gamma_{I}$ splitting the square

$$
\begin{array}{ccc}
S_{\text {red }} & \rightarrow & C^{I} \\
\downarrow & & \downarrow \\
S & \rightarrow & D^{I}
\end{array}
$$

into two commutative triangles, because $\pi^{I}$ is étale. We have a Cartesian square

$$
\begin{array}{ccc}
\Gamma\left(\alpha_{I}\right) & \rightarrow & S_{\text {red }} \times C \\
\downarrow & & \downarrow \\
\Gamma\left(\gamma_{I}\right) & \rightarrow & S \times C,
\end{array}
$$

which implies that we have a morphism of ringed spaces $i:\left(\Gamma\left(\alpha_{I}\right), \mathcal{K}_{\alpha_{I}}^{\sqrt{ }}\right) \rightarrow$ $\left(\Gamma\left(\gamma_{I}\right), \mathcal{K}_{\gamma_{I}}^{\sqrt{2}}\right)$ with nilpotent kernel. This map fits into a diagram of ringed spaces

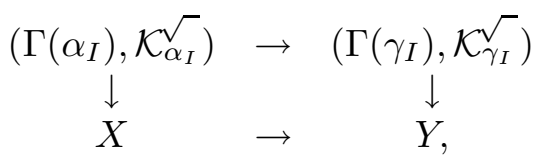

where the left vertical arrow is $\rho_{\alpha}:\left(\Gamma\left(\alpha_{I}\right), \mathcal{K}_{\alpha_{I}}^{\sqrt{-}}\right) \rightarrow X$, the right vertical arrow is the composition of $j:\left(\Gamma\left(\gamma_{I}\right), \mathcal{K}_{\gamma_{I}}^{\sqrt{ }}\right) \rightarrow\left(\Gamma\left(\beta_{I}\right), \mathcal{K}_{\beta_{I}}^{\sqrt{ }}\right)$ and $\rho_{\beta}:\left(\Gamma\left(\beta_{I}\right), \mathcal{K}_{\beta_{I}}^{\sqrt{ }}\right) \rightarrow Y$, the topological map underlying $j$ is $j_{\text {top }}=\left(\operatorname{Id}_{S} \times \pi^{I}\right): \Gamma\left(\gamma_{I}\right) \rightarrow \Gamma\left(\beta_{I}\right)$ and the structure morphism $j_{\text {top }}^{*}: \mathcal{K}_{\beta_{I}}^{\sqrt{ }} \rightarrow \mathcal{K}_{\gamma_{I}}^{\sqrt{ }}$ is an isomorphism. Note that $i$ yields an isomorphism of underlying topological spaces. Let $p$ be any point in $\Gamma\left(\alpha_{I}\right)$. We have a diagram of stalks

$$
\begin{array}{ccc}
\left(\mathcal{K}_{\alpha_{I}}^{\sqrt{2}}\right)_{p} & \leftarrow & \left(\mathcal{K}_{\gamma_{I}}^{\sqrt{ }}\right)_{p} \\
\uparrow & & \uparrow \\
\mathcal{O}_{X, \rho_{\alpha}(p)} & \leftarrow \mathcal{O}_{Y, \phi \rho_{\alpha}(p)}
\end{array}
$$

where the upper horizontal map is $i^{*}$, hence has a nilpotent kernel, and the lower horizontal map is $\phi^{*}$, hence is étale. Therefore there is a unique morphism of rings $\mathcal{O}_{X, \rho_{\alpha}(p)} \rightarrow\left(\mathcal{K}_{\gamma_{I}}^{\sqrt{ }}\right)_{p}$ for each $p$. Theses morphisms give a morphism of sheaves of rings $\rho_{\gamma, \text { top }}^{-1} \mathcal{O}_{X} \rightarrow \mathcal{K}_{\gamma_{I}}^{\sqrt{-}}$, i.e. a desired morphism of ringed spaces $\gamma$.

(3.7) Example : the cases $C=\mathbb{A}^{1}$ and $X=\mathbb{A}^{d}$. Let $C=\operatorname{Spec} k[t]$. If $S=\operatorname{Spec} R$, a morphism $f_{I}=\left(f_{i}\right): S \rightarrow C^{I}$ is the same as a collection of elements $b_{i}=f_{i}^{*}(t) \in R$. Assume these have been fixed. Then the subscheme $u_{I}^{n}\left(f_{I}\right) \subset S \times C$ from the proof of Proposition 3.4.1 is described explicitly :

$$
u_{I}^{n}\left(f_{I}\right)=\operatorname{Spec}\left(R[t] / \prod_{i \in I}\left(t-b_{i}\right)^{n+1}\right) .
$$

This implies the following. 
(3.7.2) Proposition. (a) The ring

$$
H^{0}\left(\Gamma\left(f_{I}\right), \mathcal{O}_{f_{I}}^{\wedge}\right)=\lim _{n}\left(R[t] / \prod_{i}\left(t-b_{i}\right)^{n+1}\right)
$$

is identified with the set of series $\sum_{l=0}^{\infty} a_{l}(t) \prod_{i}\left(t-b_{i}\right)^{l}$, where $a_{l}(t) \in R[t]$ are polynomials of degree less than $|I|$ (such polynomials form a set of representatives for $\left.R[t] / \prod_{i}\left(t-b_{i}\right)\right)$.

(b) The ring $H^{0}\left(\Gamma\left(f_{I}\right), \mathcal{K}_{f_{I}}\right)$ is identified with the set of series $\sum_{l \gg-\infty} a_{l}(t) \prod_{i}(t-$ $\left.b_{i}\right)^{l}$, where $a_{l}(t)$ are as in $(a)$.

(c) The subring $H^{0}\left(\Gamma\left(f_{I}\right), \mathcal{K}_{f_{I}}^{\sqrt{ }}\right)$ is identified with the series as in (b) but with the condition that all the coefficients of the polynomials $a_{l}(t), l<0$, are nilpotent elements of $R$.

(3.7.3) Notation. For fixed $b_{i} \in R$ and $a \in H^{0}\left(\Gamma\left(f_{I}\right), \mathcal{K}_{f_{I}}\right)$ we denote by $a_{l}(t) \in$ $R[t]$ the $l$ th coefficient of the series corresponding to $a$ by Proposition 3.7.2(b) and by $a_{l \nu} \in R, \nu=0, \ldots,|I|-1$, the $\nu$ th coefficient of the polynomial $a_{l}(t)$.

Assume moreover that $X=\mathbb{A}^{d}$ with coordinates $x_{1}, \ldots, x_{d}$. In this case we can give a completely explicit description of the ind-schemes $\tilde{\mathcal{L}}\left(\mathbb{A}^{d}\right)_{\mathbb{A}^{I}}$ and $\mathcal{L}\left(\mathbb{A}^{d}\right)_{\mathbb{A}^{I}}$, using Proposition 3.7.2. Indeed,

$$
\tilde{\lambda}_{\mathbb{A}^{d}, \mathbb{A}^{I}}(R)=\left\{\left(b_{i}, \rho\right) \mid\left(b_{i}\right) \in R^{I}, \rho: k[x] \rightarrow H^{0}\left(\Gamma\left(f_{I}\right), \mathcal{K}_{f_{I}}\right)\right\} .
$$

The algebra homomorphism $\rho$ is uniquely determined by the choice of

$$
\rho\left(x_{j}\right)=\sum_{l \gg-\infty} \sum_{\nu=0}^{|I|-1} a_{l \nu}^{(j)} t^{\nu} \prod_{i \in I}\left(t-b_{i}\right)^{l}, \quad a_{l \nu}^{(j)} \in R .
$$

A choice of $\rho\left(x_{j}\right)$ is the same as a choice of elements $a_{l \nu}^{(j)} \in R$. Thus the universal case corresponds to $b_{i}, a_{l \nu}^{(j)}$, with $i \in I, j \in[1, d], l \in \mathbb{Z}$ and $\nu \in[0,|I|-1]$, being independent variables, i.e.

$$
\tilde{\mathcal{L}}\left(\mathbb{A}^{d}\right)_{\mathbb{A}^{I}}=\lim _{N} \operatorname{Spec} k\left[b_{i}, a_{l \nu}^{(j)} ; l \geq-N\right] .
$$

and

$$
\mathcal{L}\left(\mathbb{A}^{d}\right)_{\mathbb{A}^{I}}=\lim _{\longrightarrow} \operatorname{Spf} k\left[b_{i}, a_{l \nu}^{(j)} ; l \geq 0\right]\left[\left[a_{l \nu}^{(j)} ;-N \leq l \leq-1\right]\right] .
$$

Notice further that

$$
\mathcal{L}_{n}^{0}\left(\mathbb{A}^{d}\right)_{\mathbb{A}^{I}}=\operatorname{Spec} k\left[b_{i}, a_{l \nu}^{(j)} ; 0 \leq l \leq n\right]
$$

In particular, we have a natural morphism

$$
\theta_{\mathbb{A}^{d}, I}: \mathcal{L}\left(\mathbb{A}^{d}\right)_{\mathbb{A}^{I}} \rightarrow \mathcal{L}_{0}^{0}\left(\mathbb{A}^{d}\right)_{\mathbb{A}^{I}}
$$

taking all $a_{l \nu}^{(j)}, l \neq 0$, to 0 . 
(3.7.7) Proposition. If $X, C$ are affine and $\phi: X \rightarrow \mathbb{A}^{d}, \pi: C \rightarrow \mathbb{A}^{1}$ are étale morphisms, there is a Cartesian diagram

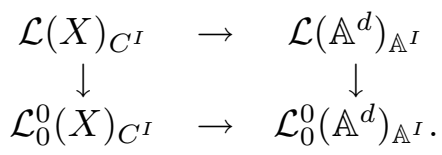

Proof : The proof is similar to that of Proposition 2.6.1.(b). Consider the diagram

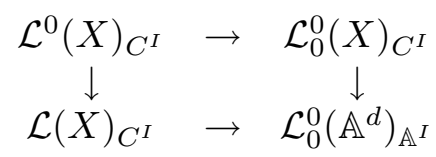

where the right vertical arrow is $\mathcal{L}_{0}^{0}(\phi)_{\pi^{I}}$, the lower horizontal arrow is the composition of $\theta_{\mathbb{A}^{d}, I}: \mathcal{L}\left(\mathbb{A}^{d}\right)_{\mathbb{A}^{I}} \rightarrow \mathcal{L}_{0}^{0}\left(\mathbb{A}^{d}\right)_{\mathbb{A}^{I}}$ defined in (3.7.6) and $\mathcal{L}(\phi)_{\pi^{I}}: \mathcal{L}(X)_{C^{I}} \rightarrow$ $\mathcal{L}\left(\mathbb{A}^{d}\right)_{\mathbb{A}^{I}}$. The upper horizontal arrow is the natural projection of $\mathcal{L}^{0}(X)_{C^{I}}$ to the 0 -th term of the projective system, see (3.4). Further, the left vertical arrow is an inductive limit of nilpotent embeddings of schemes, while the right vertical arrow is étale by (3.4.6). Therefore there exists a unique morphism $\theta_{\phi, \pi^{I}}: \mathcal{L}(X)_{C^{I}} \rightarrow$ $\mathcal{L}_{0}^{0}(X)_{C^{I}}$ splitting the diagram into two commutative triangles.

Combining $\theta_{\phi, \pi^{I}}$ with $\mathcal{L}(\phi)_{\pi^{I}}: \mathcal{L}(X)_{C^{I}} \rightarrow \mathcal{L}\left(\mathbb{A}^{d}\right)_{\mathbb{A}^{I}}$ we obtain a morphism

$$
\psi: \mathcal{L}(X)_{C^{I}} \rightarrow \mathcal{L}_{0}^{0}(X)_{C^{I}} \times_{\mathcal{L}_{0}^{0}\left(\mathbb{A}^{d}\right)_{\mathbb{A}^{I}}} \mathcal{L}\left(\mathbb{A}^{d}\right)_{\mathbb{A}^{I}}
$$

We claim that $\psi$ is an isomorphism and to prove this we construct its inverse $\chi$. Let $S$ be a scheme. A morphism from $S$ to the RHS of (3.7.8) is a compatible pair

$$
\left(\alpha: S \rightarrow \mathcal{L}\left(\mathbb{A}^{d}\right)_{\mathbb{A}^{I}}, \beta: S \rightarrow \mathcal{L}_{0}^{0}(X)_{C^{I}}\right)
$$

We construct a morphism $\chi(\alpha, \beta): S \rightarrow \mathcal{L}(X)_{C^{I}}$. For this notice that $\alpha\left(S_{\text {red }}\right) \subset$ $\mathcal{L}^{0}\left(\mathbb{A}^{d}\right)_{\mathbb{A}^{I}}$ and $\beta\left(S_{\text {red }}\right) \subset \mathcal{L}_{0}^{0}(X)_{C^{I}}$. By Proposition 3.4.6.(b) we have a map $\gamma$ : $S_{\text {red }} \rightarrow \mathcal{L}^{0}(X)_{C^{I}}$. The composition $\tilde{\gamma}$ of $\gamma$ and the embedding $\mathcal{L}^{0}(X)_{C^{I}} \rightarrow \mathcal{L}(X)_{C^{I}}$ gives a commutative diagram

$$
\begin{array}{ccc}
S_{\text {red }} & \rightarrow & \mathcal{L}(X)_{C^{I}} \\
\downarrow & & \downarrow \\
S & \rightarrow & \mathcal{L}\left(\mathbb{A}^{d}\right)_{\mathbb{A}^{I}}
\end{array}
$$

Because $\mathcal{L}(\phi)_{\pi^{I}}$ is formally etale by $(3.6 .2)(\mathrm{a})$, we set $\chi(\alpha, \beta)$ to be the unique splitting of the diagram into two exact triangles. The verification that $\chi$ is inverse to $\psi$ is straightforward. 
(3.8) The global loop space as an ind-object. We first consider the case $X=\mathbb{A}^{d}, C=\mathbb{A}^{1}$, employing the notations of (3.7). Let $\varepsilon=\left(\varepsilon_{-1}, \varepsilon_{-2}, \ldots\right) \in \mathbb{E}$ be as in (2.6). Define the scheme

$$
\mathcal{L}^{\varepsilon}\left(\mathbb{A}^{d}\right)_{\mathbb{A}^{I}}=\operatorname{Spec}\left(k\left[b_{i}, a_{l \nu}^{(j)}\right] /\left(a_{l, \nu_{1}}^{(j)} \cdots a_{l, \nu_{1+\varepsilon_{l}}}^{(j)} ; l<0\right)\right),
$$

where $j \in[1, d], l \in \mathbb{Z}$, and $\nu_{1}, \ldots, \nu_{1+\varepsilon_{l}} \in[0,|I|-1]$ are arbitrary for $l<0$. It is clear that

$$
\mathcal{L}\left(\mathbb{A}^{d}\right)_{\mathbb{A}^{I}}=\lim _{\varepsilon} \mathcal{L}^{\varepsilon}\left(\mathbb{A}^{d}\right)_{\mathbb{A}^{I}}
$$

next, assume that $X$ is an arbitrary smooth scheme of finite type and $C$ is an arbitrary smooth curve. Then $X$ can be covered by open $U=\operatorname{Spec} A$ possessing an étale map $\phi: U \rightarrow \mathbb{A}^{d}$ and similarly $C$ can be covered by open $V$ possessing an étale $\pi: V \rightarrow \mathbb{A}^{1}$. We set

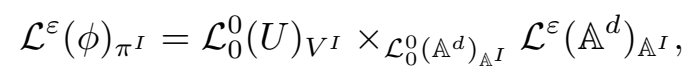

where the map $\mathcal{L}^{\varepsilon}\left(\mathbb{A}^{d}\right)_{\mathbb{A}^{I}} \rightarrow \mathcal{L}_{0}^{0}\left(\mathbb{A}^{d}\right)_{\mathbb{A}^{I}}$ is the restriction of $\theta_{\mathbb{A}^{d}, I}$ defined in (3.7.6). This is an affine scheme.

(3.8.2) Proposition. The ind-object " $\lim _{\varepsilon} " \mathcal{L}^{\varepsilon}(\phi)_{\pi^{I}}$ in $\mathbf{S c h}$ is isomorphic to $\mathcal{L}(U)_{V^{I}}$.

Proof: This follows from the case $X=\mathbb{A}^{d}, C=\mathbb{A}^{1}$, from Proposition 3.7.7, and from the fact that fiber products commute with filtering inductive limits.

(3.9) The global loop space as an ind-pro-object. We keep the notation of (3.8). For $\varepsilon \in \mathbb{E}, n \geq 1$ consider the scheme

$$
\mathcal{L}_{n}^{\varepsilon}\left(\mathbb{A}^{d}\right)_{\mathbb{A}^{I}}=\operatorname{Spec}\left(k\left[b_{i}, a_{l \nu}^{(j)} ; l \leq n\right] /\left(a_{l \nu_{1}}^{(j)} \cdots a_{l, \nu_{1+\varepsilon_{l}}}^{(j)}\right)\right) .
$$

We set

$$
\mathcal{L}_{n}^{\varepsilon}(\phi)_{\pi^{I}}=\mathcal{L}_{0}^{0}(U)_{V^{I}} \times{ }_{\mathcal{L}_{0}^{0}\left(\mathbb{A}^{d}\right)_{\mathbb{A}^{I}}} \mathcal{L}_{n}^{\varepsilon}\left(\mathbb{A}^{d}\right)_{\mathbb{A}^{I}}
$$

where the map $\mathcal{L}_{n}^{\varepsilon}\left(\mathbb{A}^{d}\right)_{\mathbb{A}^{I}} \rightarrow \mathcal{L}_{0}^{0}\left(\mathbb{A}^{d}\right)_{\mathbb{A}^{I}}$ is defined as $\theta_{\mathbb{A}^{d}, I}$ in (3.7.6).

(3.9.3) Proposition. (a) The scheme $\mathcal{L}_{n}^{\varepsilon}(\phi)_{\pi^{I}}$ is of finite type and is a nilpotent extension of $\mathcal{L}_{n}^{0}(U)_{V^{I}}$. The second projection $\mathcal{L}_{n}^{\varepsilon}(\phi)_{\pi^{I}} \rightarrow \mathcal{L}_{n}^{\varepsilon}\left(\mathbb{A}^{d}\right)_{\mathbb{A}^{I}}$ is étale. Moreover $\mathcal{L}^{\varepsilon}(\phi)_{\pi^{I}}=\lim _{n} \mathcal{L}_{n}^{\varepsilon}(\phi)_{\pi^{I}}$.

(b) The schemes $\left.\overleftarrow{\mathcal{L}_{n}^{\varepsilon}(\phi)}\right)_{\pi^{I}}$ form a double ind-pro-system with Cartesian squares $\left(n^{\prime} \geq n, \varepsilon \leq \varepsilon^{\prime}\right)$

$$
\begin{array}{cccc}
\mathcal{L}_{n^{\prime}}^{\varepsilon}(\phi)_{\pi^{I}} & \hookrightarrow & \mathcal{L}_{n^{\prime}}^{\varepsilon^{\prime}}(\phi)_{\pi^{I}} \\
\downarrow & & \downarrow \\
\mathcal{L}_{n}^{\varepsilon}(\phi)_{\pi^{I}} & \hookrightarrow & \mathcal{L}_{n}^{\varepsilon^{\prime}}(\phi)_{\pi^{I}}
\end{array}
$$


where the vertical arrows are smooth affine morphisms.

(c) The ind-pro-object " $\mathrm{lim}_{\varepsilon} "{ }^{-\lim _{n}} " \mathcal{L}_{n}^{\varepsilon}(\phi)_{\pi^{I}}$ is independent, up to isomorphism, on $\phi, \pi$.

Proof: The proof of $(a)$ is similar to that of Proposition 3.8.2. In order to prove that $\mathcal{L}_{n}^{\varepsilon}(\phi)_{\pi^{I}} \rightarrow \mathcal{L}_{n}^{\varepsilon}\left(\mathbb{A}^{d}\right)_{\mathbb{A}^{I}}$ is étale, due to (3.9.2) it is sufficient to check that the morphism $\mathcal{L}_{0}^{0}(\phi)_{\pi^{I}}: \mathcal{L}_{0}^{0}(U)_{V^{I}} \rightarrow \mathcal{L}_{0}^{0}\left(\mathbb{A}^{d}\right)_{\mathbb{A}^{I}}$ is étale. This is a consequence of Proposition (3.4.6)(a).

Claim (b) is obvious in the case $\phi=\mathrm{Id}, \pi=\mathrm{Id}$. The general case follows from (3.9.2) since the base change of a smooth affine morphism is still smooth affine, and the base change of a Cartesian square is Cartesian.

(c) The pro-object " $\longleftarrow_{n} " \mathcal{L}_{n}^{\varepsilon}(\phi)_{\pi^{I}}$ in $\operatorname{Pro}\left(\mathbf{A f f}^{\mathrm{ft}}\right)$ can be identified, due to Proposition 2.1.2, with the scheme $\mathcal{L}^{\varepsilon}(\phi)_{\pi^{I}}$. The ind-object "lim ${ }_{\varepsilon} \mathcal{L}^{\varepsilon}(\phi)_{\pi^{I}} \operatorname{in} \operatorname{Ind}\left(\operatorname{Pro}\left(\mathbf{A f f}{ }^{\mathrm{ft}}\right)\right)$ can then be identified with the ind-scheme $\mathcal{L}(U)_{V^{I}}$. 


\section{D-MODUleS OVER IND-SCHEMES}

(4.1) Reminder on $\mathcal{D}$-modules. From now on we assume that $\operatorname{char}(k)=0$. Fix a $k$-scheme $S$ of finite type. Let $\mathbf{S c h}_{S}^{\mathrm{ft}}$ denote the category of $S$-schemes of finite type. For any such scheme $X$ let $\mathbf{O}_{X}$ be the category of all quasi-coherent $\mathcal{O}_{X^{-}}$ modules. For a morphism $f: X \rightarrow Y$ in $\mathbf{S c h}_{S}^{\mathrm{ft}}$ we denote by $f_{*}, f^{*}$ the functors of the direct and inverse images on $\mathbf{O}_{X}, \mathbf{O}_{Y}$. If $f$ is a closed embedding and $\mathcal{E} \in \mathbf{O}_{Y}$, let

$$
f^{!} \mathcal{E}=f^{-1} \mathcal{H o m}_{\mathcal{O}_{Y}}\left(f_{*} \mathcal{O}_{X}, \mathcal{E}\right)
$$

be the inverse image of the subsheaf of $\mathcal{E}$ consisting of sections supported schemetheoretically on $f(X) \subset Y$.

For $X \in \mathbf{S c h}_{S}^{\mathrm{ft}}$ let $\mathbf{D}_{X / S}$ be the category of coherent right $\mathcal{D}_{X / S}$-modules on $X$. It is defined as follows, see [BD2, §7.10] or [G, §0.2.2]. If $X$ is smooth over $S$, then we have the sheaf of rings $\mathcal{D}_{X / S}$ of differential operators from $\mathcal{O}_{X}$ to itself which are linear over $\mathcal{O}_{S}$. An object of $\mathbf{D}_{X / S}$ is then a coherent sheaf of right $\mathcal{D}_{X / S}$-modules. It is quasicoherent over $\mathcal{O}_{X}$. Next, if $X$ admits a closed embedding into a smooth $S$ scheme $Y$, one defines $\mathbf{D}_{X / S}$ as $\mathbf{D}_{Y / S, X}$, the full subcategory of $\mathbf{D}_{Y / S}$ consisting of modules supported (as sheaves) on $X$. This definition is independent on the choice of embedding: if $X$ is embedded into two smooth $S$-schemes $Y_{1}$ and $Y_{2}$, then one has an equivalence $\mathbf{D}_{Y_{1} / S, X} \rightarrow \mathbf{D}_{Y_{2} / S, X}$ which is unique up to a unique isomorphism of functors. Now, given any $X$, an embedding into a smooth scheme always exists locally on $X$. Therefore we have an open covering $X=\bigcup U_{\alpha}$, the categories $\mathbf{D}_{U_{\alpha} / S}, \mathbf{D}_{U_{\alpha \beta} / S}$ etc. and the obvious restriction functors among them. One then defines an object $\mathcal{M}$ of $\mathbf{D}_{X / S}$ as a collection of objects $\mathcal{M}_{\alpha} \in \mathbf{D}_{U_{\alpha} / S}$ together with isomorphisms of their images in $\mathbf{D}_{U_{\alpha \beta} / S}$ whose images in $\operatorname{Mor}\left(\mathbf{D}_{U_{\alpha \beta \gamma} / S}\right)$ satisfy the obvious compatibility conditions.

Given $X \in \mathbf{S c h}_{S}^{\mathrm{ft}}$ and $\mathcal{M} \in \mathbf{D}_{X / S}$, we define a sheaf $\mathcal{M}^{\mathcal{O}} \in \mathbf{O}_{X}$ as follows. If $X$ admits a closed embedding $i: X \rightarrow Y$ with $Y$ smooth over $S$ and $\mathcal{M}$ is represented by a sheaf of right $\mathcal{D}_{Y / S}$-modules supported on $X$ (which we also denote $\mathcal{M}$ ) then we set $\mathcal{M}^{\mathcal{O}}=i^{!} \mathcal{M}$. This definition is easily seen to be independent on the choice of $Y$. In the case of a general $X$ one defines $\mathcal{M}^{\mathcal{O}}$ by gluing the sheaves given by above procedures on open parts of $X$ admitting embeddings into smooth schemes.

Any smooth morphism $f: X \rightarrow Y$ in $\mathbf{S c h}_{S}^{\mathrm{ft}}$ induces the functor of inverse image $f^{\bullet}: \mathbf{D}_{Y / S} \rightarrow \mathbf{D}_{X / S}$. If $\omega_{X / Y}$ is the relative canonical bundle, then

$$
f^{\bullet}(\mathcal{M})^{\mathcal{O}}=f^{*}\left(\mathcal{M}^{\mathcal{O}}\right) \otimes_{\mathcal{O}_{X}} \omega_{X / Y}
$$

We have then a canonical embedding

$$
\mathcal{M}^{\mathcal{O}} \hookrightarrow f_{*}\left(\left(f^{\bullet} \mathcal{M}\right)^{\mathcal{O}} \otimes \omega_{X / Y}^{-1}\right)
$$


If $f: X \rightarrow Y$ is a closed embedding in $\mathbf{S c h}_{S}^{\mathrm{ft}}$, we have an exact functor of direct image $f_{\bullet}: \mathbf{D}_{X / S} \rightarrow \mathbf{D}_{Y / S}$. In the particular case when $X, Y$ are smooth over $S$, we can view $\mathcal{M} \in \mathbf{D}_{X / S}$ as a sheaf on $X$ and we have

$$
f_{\bullet}(\mathcal{M})=f_{*}\left(\mathcal{M} \otimes_{\mathcal{D}_{X / S}} \mathcal{D}_{X \rightarrow Y}\right) .
$$

Here $\mathcal{D}_{X \rightarrow Y}$ is the sheaf of differential operators from $f^{-1} \mathcal{O}_{Y}$ to $\mathcal{O}_{X}$ linear over $\mathcal{O}_{S}$, see $[\mathrm{BB}],[\mathrm{G}]$.

For a general closed embedding $f$ we have a canonical embedding

$$
\mathcal{M}^{\mathcal{O}} \simeq f^{!} f_{\bullet}(\mathcal{M}) \hookrightarrow f^{-1} f_{\bullet}(\mathcal{M}) .
$$

For example, if $X$ is non-reduced and $i: X_{\text {red }} \hookrightarrow X$ is the reduced part, then $i \bullet$ identifies $\mathbf{D}_{X_{\text {red }} / S}$ with $\mathbf{D}_{X / S}$.

We have the following base change property.

(4.1.4) Lemma. Suppose that in a Cartesian diagram of S-schemes of finite type

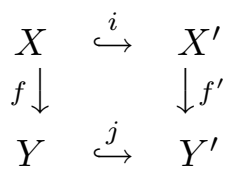

the morphisms $f, f^{\prime}$ are smooth, $i, j$ are closed embeddings. Then for any $\mathcal{M} \in$ $\mathbf{D}_{Y / S}$, we have

$$
i_{\bullet} f^{\bullet} \mathcal{M} \simeq f^{\prime \bullet} j_{\bullet} \mathcal{M} \text {. }
$$

Note that if $f$ is a closed embedding of smooth schemes over $S$, the inverse image functor $f^{\bullet}$ is still defined. Furthermore, the projection formula holds for right $\mathcal{D}$ modules. More precisely, if $f$ is a closed or open embedding of smooth $S$-schemes and $\mathcal{M} \in \mathbf{D}_{X / S}, \mathcal{N} \in \mathbf{D}_{Y / S}$, then there is a canonical isomorphism

$$
f_{\bullet}\left(f^{\bullet}(\mathcal{N}) \otimes_{\mathcal{O}_{X}} \mathcal{M} \otimes_{\mathcal{O}_{X}} \omega_{X}^{-1}\right) \simeq \mathcal{N} \otimes_{\mathcal{O}_{Y}} f_{\bullet}(\mathcal{M}) \otimes_{\mathcal{O}_{Y}} \omega_{Y}^{-1}
$$

\section{(4.2) $\mathcal{D}$-modules over pro-schemes.}

(4.2.1) Definition. (cf. [Kap].) Let $A$ be a filtering poset and $\left(\mathbf{C}_{\alpha}\right)_{\alpha \in A}$ be an inductive system of categories labelled by $A$. In other words, for each $\alpha \leq \beta$ we have a functor $i_{\alpha \beta}: \mathbf{C}_{\alpha} \rightarrow \mathbf{C}_{\beta}$, for each $\alpha \leq \beta \leq \gamma$ a natural isomorphism $i_{\beta \gamma} \circ i_{\alpha \beta} \Rightarrow i_{\alpha \gamma}$ and these isomorphisms satisfy the obvious coherence conditions for any $\alpha \leq \beta \leq \gamma \leq \delta$.

The inductive limit $2 \lim _{\alpha} \mathbf{C}_{\alpha}$ is the category whose objects are pairs $\left(\alpha, x_{\alpha}\right)$, $\alpha \in A, x_{\alpha} \in \mathrm{Ob}\left(\mathbf{C}_{\alpha}\right)$ and

$$
\operatorname{Hom}\left(\left(\alpha, x_{\alpha}\right),\left(\beta, y_{\beta}\right)\right)=\lim _{\longrightarrow} \operatorname{Hom}_{\mathbf{C}_{\alpha}}\left(i_{\alpha \gamma}\left(x_{\alpha}\right), i_{\beta \gamma}\left(y_{\beta}\right)\right) .
$$


(4.2.2) Definition. A $S$-scheme $X_{\infty}$ (possibly of infinite type) is called compact if it can be represented as $\lim _{\alpha} X_{\alpha}$ where $\left(X_{\alpha}\right)_{\alpha \in A}$ is a filtering projective system over $\mathbf{S c h}_{S}^{\mathrm{ft}}$ such that all the maps $p_{\alpha \beta}: X_{\beta} \rightarrow X_{\alpha}, \alpha \leq \beta$, are affine morphisms.

(4.2.3) Proposition. A scheme is compact if and only if it is quasi-compact and quasi-separated. The category of compact $k$-schemes can be identified with a full subcategory in $\operatorname{Pro}\left(\mathbf{S c h}_{S}^{\mathrm{ft}}\right)$, via $X_{\infty}=\lim _{\alpha} X_{\alpha} \mapsto \stackrel{\longleftarrow^{-}}{\longleftarrow_{\alpha}} " X_{\alpha}$.

Proof: This follows from [TT], Appendix C, Theorem C9.

(4.2.4) Definition. (a) A compact $S$-scheme $X_{\infty}$ is called smooth if it can be represented as $\lim _{\alpha} X_{\alpha}$ for some $\left(X_{\alpha}\right)$ as in (4.2.2) with the extra property that each $X_{\alpha}$ is a smooth $S$-scheme and each $p_{\alpha \beta}$ is a smooth affine morphism.

(b) $X_{\infty}$ is called almost smooth if it can be represented as $\lim X_{\alpha}$ for some $\left(X_{\alpha}\right)$ as in (4.2.2) with the extra property that each $p_{\alpha \beta}$ is a smooth affine morphism.

(4.2.5) Example. For any smooth $X \in \mathbf{S c h}_{k}^{\mathrm{ft}}$, the scheme $\mathcal{L}^{0}(X)$ is smooth and compact over $\operatorname{Spec}(k)$, and the scheme $\mathcal{L}^{0}(X)_{C^{I}}$ is smooth and compact over $C^{I}$. Moreover, the schemes $\mathcal{L}^{\varepsilon}(\phi), \mathcal{L}^{\varepsilon}(\phi)_{\pi^{I}}$ from (2.6.3) and (3.8) are almost smooth.

Let $q: X_{\infty} \rightarrow S$ be a compact almost smooth $S$-scheme and $\left(q_{\alpha}: X_{\alpha} \rightarrow S\right)$ be as in Definition 4.2.4. We have two inductive systems of categories $\left(\mathbf{O}_{X_{\alpha}}, p_{\alpha \beta}^{*}\right)$, $\left(\mathbf{D}_{X_{\alpha} / S}, p_{\alpha \beta}^{\bullet}\right)$. We set $\mathbf{D}_{X_{\infty} / S}=2 \underset{\lim }{\longrightarrow} \mathbf{D}_{X_{\alpha} / S}$.

(4.2.6) Proposition. The category $\mathbf{D}_{X_{\infty} / S}$ is independent, up to canonical equivalence of categories, on the choice of $\left(X_{\alpha}\right)$ as in (4.2.4).

Proof : We first consider the case when $X_{\infty}$ is smooth, so that each $X_{\alpha}$ is smooth over $S$. Let $\mathbf{D}_{X_{\alpha} / S}^{\ell}$ be the category of left coherent $\mathcal{D}_{X_{\alpha} / S}$-modules. We have an equivalence

$$
\mathbf{D}_{X_{\alpha} / S}^{\ell} \rightarrow \mathbf{D}_{X_{\alpha} / S}, \mathcal{N} \mapsto \mathcal{N} \otimes \omega_{X_{\alpha} / S}
$$

Let also $\mathcal{D}_{X_{\infty} / S}$ be the sheaf of rings of differential operators on $X_{\infty}$ linear over $\mathcal{O}_{S}$. It is equipped with the natural topology, see $[\mathrm{KT}], \S 1.7$. Using the pullback of left $\mathcal{D}$-modules (which is the same as for quasi-coherent sheaves) we get an inductive system of categories $\mathbf{D}_{X_{\alpha} / S}^{\ell}$. It is proved in $[\mathrm{KT}], \S 1.9$, that $2 \lim _{\alpha} \mathbf{D}_{X_{\alpha} / S}^{\ell}$ is identified with the category of discrete, locally finitely generated quasicoherent sheaves of left $\mathcal{D}_{X_{\infty} / S}$-modules, and thus is independent on the choice of $\left(X_{\alpha}\right)$. Therefore the category $2 \lim _{\alpha} \mathbf{D}_{X_{\alpha} / S}$, being equivalent to the previous one, is also independent.

Now assume that $X_{\infty}$ is almost smooth. Fix $\alpha_{0} \in A$. Using a covering of $X_{\alpha_{0}}$ by affine open subsets, we reduce to the case when $X_{\alpha_{0}}$ (and thus $X_{\infty}$ ) is affine. We can also assume that $\alpha_{0}$ is the minimal element in $A$. Let us embed $X_{\alpha_{0}}$ as 
a closed subscheme into a smooth affine $S$-scheme $Y_{\alpha_{0}}$. We can then extend each $p_{\alpha_{0}, \alpha}: X_{\alpha} \rightarrow X_{\alpha_{0}}$ to a smooth map $q_{\alpha_{0}, \alpha}: Y_{\alpha} \rightarrow Y_{\alpha_{0}}$. We get then a smooth compact scheme $Y_{\infty}=\lim _{\leftarrow} Y_{\alpha}$ containing $X_{\infty}$ as a closed subscheme. The category $\underset{\longrightarrow \alpha}{2} \mathbf{D}_{X_{\alpha} / S}$ is then identified with the category of sheaves of discrete locally finitely generated left $\mathcal{D}_{Y_{\infty} / S}$-modules supported on $X_{\infty}$.

Informally, an object of $\mathbf{D}_{X_{\infty} / S}$ is a " $\mathcal{D}$-module pulled back from some $X_{\alpha}$ ". Let $p_{\alpha}: X_{\infty} \rightarrow X_{\alpha}$ be the projection. Let $p_{\alpha}^{\bullet}: \mathbf{D}_{X_{\alpha} / S} \rightarrow \mathbf{D}_{X_{\infty} / S}, \mathcal{M}_{\alpha} \mapsto\left(\alpha, \mathcal{M}_{\alpha}\right)$ be the canonical functor. If the compact $S$-scheme $X_{\infty}$ is smooth, there is the functor

$\mathbf{D}_{X_{\infty} / S} \rightarrow \underset{2 \lim }{\lim } \mathbf{O}_{X_{\alpha}} \subset \mathbf{O}_{X_{\infty}}, \quad p_{\alpha}^{\bullet} \mathcal{M}_{\alpha} \mapsto\left(p_{\alpha}^{\bullet} \mathcal{M}_{\alpha}\right)^{\mathcal{O}}:=\left(\left(p_{\alpha \beta}^{\bullet} \mathcal{M}_{\alpha}\right)^{\mathcal{O}} \otimes_{\mathcal{O}_{X_{\beta}}} \omega_{X_{\beta}}^{-1}\right)_{\beta \geq \alpha}$.

In particular, we can associate to any $\mathcal{M} \in \mathbf{D}_{X_{\infty} / S}$ its "space of global sections", i.e., the direct image to $S$ as an $\mathcal{O}$-module. This is a quasi-coherent sheaf on $S$ such that if $\mathcal{M}=p_{\alpha}^{\bullet} \mathcal{M}_{\alpha}$ then

$$
q_{*}(\mathcal{M}):=q_{*}\left(\mathcal{M}^{\mathcal{O}}\right)=\lim _{\beta \geq \alpha}\left(q_{\beta}\right)_{*}\left(X_{\beta},\left(p_{\alpha \beta}^{\bullet} \mathcal{M}_{\alpha}\right)^{\mathcal{O}} \otimes_{\mathcal{O}_{X_{\beta}}} \omega_{X_{\beta}}^{-1}\right)
$$

When $S=$ Spec $k$, we write $\Gamma\left(X_{\infty}, \mathcal{M}\right)$ for $q_{*}(\mathcal{M})$.

(4.3) $\mathcal{D}$-modules over ind-schemes.

(4.3.1) Definition. Let $A$ be a filtering poset and $\left(\mathbf{C}_{\alpha}\right)_{\alpha \in A}$ be a projective system of categories labelled by $A$. In other words, for each $\alpha \leq \beta$ we have a functor $j_{\alpha \beta}: \mathbf{C}_{\beta} \rightarrow \mathbf{C}_{\alpha}$ and for any $\alpha \leq \beta \leq \gamma$ a natural isomorphism $j_{\alpha \beta} \circ j_{\beta \gamma} \Rightarrow j_{\alpha \gamma}$ satisfying the obvious compatibility conditions.

The projective limit $2 \lim \mathbf{C}_{\alpha}$ is the category whose objects are systems consisting of objects $x_{\alpha} \in \mathbf{C}_{\alpha}$ given for all $\alpha \in A$ and isomorphisms $j_{\alpha \beta}\left(x_{\beta}\right) \rightarrow x_{\alpha}$ given for each $\alpha \leq \beta$ and satisfying the compatibility condition for each $\alpha \leq \beta \leq \gamma$. Morphisms are defined in the obvious way.

(4.3.2) Definition. Let $X^{\infty}$ be an ind-S-scheme. We say that $X_{\infty}$ is discrete over $S$ if it can be represented as $X^{\infty}={ }^{\longrightarrow}{ }_{\alpha}$ " $X^{\alpha}$ where $\left(X^{\alpha}\right)_{\alpha \in A}$ is a filtering inductive system over $\mathbf{S c h}_{S}^{\mathrm{ft}}$ such that each map $i_{\alpha \beta}: X^{\alpha} \rightarrow X^{\beta}, \alpha \leq \beta$, is a closed embedding.

(4.3.3) Example. For any $\phi, U$ as in $(2.7)$ the ind-scheme $\mathcal{L}_{n}(\phi)$ is discrete over $\operatorname{Spec}(k)$.

Let $q: X^{\infty} \rightarrow S$ be a discrete ind-scheme over $S$ and $\left(q^{\alpha}: X^{\alpha} \rightarrow S\right)$ be as in Definition 4.3.2. We have then the projective system of categories $\left(\mathbf{O}_{X^{\alpha}}, i_{\alpha \beta}^{!}\right)$. We 
define $\mathbf{O}_{X^{\infty}}=2 \lim \mathbf{O}_{X^{\alpha}}$. If $\left(\mathcal{E}^{\alpha}, \gamma_{\alpha \beta}: \mathcal{E}^{\alpha} \rightarrow i_{\alpha \beta}^{!} \mathcal{E}^{\beta}\right)$ is an object of $\mathbf{O}_{X^{\infty}}$, then the direct images $q_{*}^{\alpha} \overleftarrow{\left(\mathcal{E}^{\alpha}\right)}$ form an inductive system and we define

$$
q_{*} \mathcal{E}=\lim _{\alpha} q_{*}^{\alpha}\left(\mathcal{E}^{\alpha}\right)
$$

When $S=\operatorname{Spec}(k)$ we write $\Gamma\left(X^{\infty}, \mathcal{E}\right)$ for $q_{*} \mathcal{E}$.

We will also use the category $\hat{\mathbf{O}}_{X^{\infty}}$ which is the limit of the projective system of categories $\left(\mathbf{O}_{X^{\alpha}}, i_{\alpha \beta}^{*}\right)$. There is a functor

$$
\mathbf{O}_{X^{\infty}} \times \hat{\mathbf{O}}_{X^{\infty}} \rightarrow \mathbf{O}_{X^{\infty}}, \quad(\mathcal{E}, \hat{\mathcal{F}}) \mapsto \mathcal{E} \otimes \hat{\mathcal{F}}=\left(\mathcal{E}^{\alpha} \otimes_{\mathcal{O}_{X^{\alpha}}} \hat{\mathcal{F}}^{\alpha}\right)
$$

See [BD2, §7.11.4] for more details on $\mathbf{O}_{X^{\infty}}, \hat{\mathbf{O}}_{X^{\infty}}$.

We set also $\mathbf{D}_{X^{\infty} / S}=2 \lim \left(\mathbf{D}_{X^{\alpha} / S}, i_{\alpha \beta} \bullet\right)$. Let $i_{\alpha}$ be the embedding $X^{\alpha} \hookrightarrow X^{\infty}$. Let $i_{\alpha \bullet}: \mathbf{D}_{X^{\alpha} / S} \rightarrow \mathbf{D}_{X \infty / S}, \mathcal{M}^{\alpha} \mapsto\left(\alpha, \mathcal{M}^{\alpha}\right)$ be the canonical functor. It is exact. There is also the functor

$$
\mathbf{D}_{X^{\infty} / S} \rightarrow \mathbf{O}_{X^{\infty}}, \quad \mathcal{M}=i_{\alpha \bullet} \mathcal{M}^{\alpha} \mapsto \mathcal{M}^{\mathcal{O}}=\left(i_{\alpha \bullet} \mathcal{M}^{\alpha}\right)^{\mathcal{O}}:=\left(i_{\alpha \beta \bullet} \mathcal{M}^{\alpha}\right)_{\beta \geq \alpha}^{\mathcal{O}}
$$

see (4.1.3). In particular, to any $\mathcal{M} \in \mathbf{D}_{X^{\infty} / S}$ we can associate its direct image to $S:$ if $\mathcal{M}$ is represented by $\mathcal{M}^{\alpha} \in \mathbf{D}_{X^{\alpha} / S}$, then

$$
q_{*}(\mathcal{M}):=q_{*}\left(\mathcal{M}^{\mathcal{O}}\right)=\lim _{\beta \geq \alpha} q_{*}^{\beta}\left(\left(i_{\alpha \beta} \cdot \mathcal{M}^{\alpha}\right)^{\mathcal{O}}\right) .
$$

(4.3.8) Remark. (a) We have an exact functor

$$
2 \lim _{\longrightarrow} \mathbf{O}_{X^{\alpha}} \rightarrow \mathbf{O}_{X^{\infty}}, \quad\left(\alpha, \mathcal{E}^{\alpha}\right) \mapsto\left(i_{\alpha \beta *} \mathcal{E}^{\alpha}\right)_{\beta \geq \alpha}
$$

The two categories are not equivalent in general.

(b) The category $\mathbf{O}_{X^{\infty}}$ is closed by inductive limits.

(c) If the ind-scheme $X^{\infty}$ is not discrete anymore, the $\mathcal{O}_{X^{\alpha}-\text { module }} i_{\alpha \beta}^{!} \mathcal{E}^{\beta}$ may not be quasi-coherent. However the category $\mathbf{O}_{X^{\infty}}$ is still well-defined.

Let $X^{\infty}=\lim _{\longrightarrow} X^{\alpha}$ be a formally smooth (over $S$ ) discrete ind- $S$-scheme. Following [BD2, Proposition 7.11.8] we define the tangent sheaf of $X$ to be the object $\hat{\Theta}_{X \infty / S} \in \hat{\mathbf{O}}_{X^{\infty}}$ such that

$$
\mathcal{E} \otimes \hat{\Theta}_{X^{\infty} / S}=\lim _{\alpha} \mathcal{H o m}\left(\Omega_{X^{\alpha} / S}^{1}, \mathcal{E}^{\alpha}\right), \quad \forall \mathcal{E} \in \mathbf{O}_{X^{\infty}}
$$

Denoting $i^{\alpha}: X^{\alpha} \rightarrow X^{\infty}$ the canonical embedding, we have that $i^{\alpha *} \hat{\Theta}_{X^{\infty} / S}$ is a locally free $\mathcal{O}_{X^{\alpha}}$-module (possibly of infinite rank), see [BD2, Proposition 7.12.13].

(4.4) $\mathcal{D}$-modules over ind-pro-schemes. The following definition is inspired by the paper of K. Kato [Kat]. 
(4.4.1) Definition. An ind-S-scheme $X_{\infty}^{\infty}$ is called locally compact if it can be represented as

$$
X_{\infty}^{\infty}=\lim _{\alpha \in A \longleftarrow} \lim _{\beta \in B} X_{\beta}^{\alpha}
$$

where $\left(X_{\beta}^{\alpha}\right)$ is a bi-filtering ind-pro-system over $\mathbf{S c h}_{S}^{\mathrm{ft}}$ with the following properties:

(1) For each $\beta \in B$ and $\alpha \leq \alpha^{\prime} \in A$ the structure map $i_{\beta}^{\alpha \alpha^{\prime}}: X_{\beta}^{\alpha} \rightarrow X_{\beta}^{\alpha^{\prime}}$ is a closed embedding.

(2) For each $\alpha \in A$ and $\beta \leq \beta^{\prime} \in B$ the structure map $p_{\beta \beta^{\prime}}^{\alpha}: X_{\beta^{\prime}}^{\alpha} \rightarrow X_{\beta}^{\alpha}$ is an affine morphism.

(3) For each $\alpha \leq \alpha^{\prime} \in A$ and $\beta \leq \beta^{\prime} \in B$ the commutative square

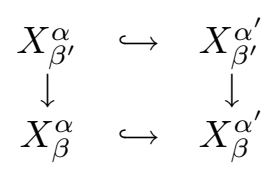

is Cartesian.

We denote by $\mathbf{L c}_{S} \subset \mathbf{I s c h}_{S}$ the full subcategory formed by locally compact ind$S$-schemes.

Let $\left(X_{\beta}^{\alpha}\right)$ be an ind-pro-system as above. The maps $p_{\beta \beta^{\prime}}^{\alpha}$ being affine, the projective limit $\lim _{\leftarrow} X_{\beta}^{\alpha}$ is represented by a compact scheme (possibly of infinite type) denoted by $X_{\infty}^{\alpha}$. Similarly, we have the discrete ind-schemes $X_{\beta}^{\infty}=\lim _{\alpha} X_{\beta}^{\alpha}$. By definition $X_{\infty}^{\infty}=\lim _{\alpha} X_{\infty}^{\alpha}$.

(4.4.2) Proposition. For an ind-pro-system satisfying the conditions of (4.4.1) we also have $X_{\infty}^{\infty}=\lim _{\leftarrow} X_{\beta}^{\infty}=\lim _{\lim _{\beta}} X_{\beta}^{\alpha}$.

Proof: By passing to the functors represented by our (ind-)schemes, we reduce the statement (a) to the following lemma whose proof we leave to the reader.

(4.4.3) Lemma. Let $\left(T_{\beta}^{\alpha}\right)_{\alpha \in A, \beta \in B}$ be a bi-filtering ind-pro-system of sets. Then there is a canonical map

$$
c: \lim _{\alpha} \lim _{\beta} T_{\beta}^{\alpha} \rightarrow \lim _{\longleftarrow} \lim _{\beta} T_{\beta}^{\alpha} .
$$

If, moreover, all the squares in $\left(T_{\beta}^{\alpha}\right)$ are Cartesian, then $c$ is an isomorphism.

(4.4.4) Definition. We say that a locally compact ind-S-scheme $X_{\infty}^{\infty}$ is smooth (over $S$ ) if it admits a presentation as in (4.1.1) where:

(1) All the $p_{\beta \beta^{\prime}}^{\alpha}$ are smooth morphisms of relative dimension $d_{\beta \beta^{\prime}}$ (independent on $\alpha)$. There is an element $(\alpha, \beta) \in A \times B$ such that $X_{\beta}^{\alpha}$ is smooth over $S$.

(2) All the ind-S-schemes $X_{\beta}^{\infty}=\lim _{\alpha} X_{\beta}^{\alpha}$ are formally smooth over $S$. 
Let $X_{\infty}^{\infty}$ be a locally compact smooth ind-S-scheme and $\left(X_{\beta}^{\alpha}\right)$ be an ind-prosystem as in (4.4.1), (4.4.4). By Lemma 4.1.4, we have then a double inductive system of categories $\left(\mathbf{D}_{X_{\beta}^{\alpha} / S}, i_{\beta \bullet^{\prime}}^{\alpha \alpha^{\prime}}, p_{\beta \beta^{\prime}}^{\alpha \bullet}\right)$ and we define the category of (right) $\mathcal{D}$ modules on $X_{\infty}^{\infty}$ to be $\mathbf{D}_{X_{\infty}^{\infty} / S}=2 \lim _{\alpha, \beta} \mathbf{D}_{X_{\beta}^{\alpha} / S}$.

(4.4.5) Proposition. The category $\mathbf{D}_{X_{\infty}^{\infty} / S}$ is independent, up to canonical equivalence, on the choice of $\left(X_{\beta}^{\alpha}\right)$ as in (4.4.4).

Proof: Each $X_{\infty}^{\alpha}=\lim _{\leftarrow} X_{\beta}^{\alpha}$ being almost smooth, the category $\mathbf{D}_{X_{\infty}^{\alpha} / S}=2 \lim _{\beta} \mathbf{D}_{X_{\beta}^{\alpha} / S}$ depends, by Proposition 4.2.6, on $X_{\infty}^{\alpha}$ only. Next, for $\alpha<\alpha^{\prime}$ the functor $\mathbf{D}_{X_{\infty}^{\alpha} / S} \rightarrow$ $\mathbf{D}_{X_{\infty}^{\alpha^{\prime}} / S}$ depends only on the morphism $X_{\infty}^{\alpha} \rightarrow X_{\infty}^{\alpha^{\prime}}$. This is seen by the same argument as in (4.2.6). Let $\left(\tilde{X}_{\tilde{\beta}}^{\tilde{\alpha}}\right)$ be another ind-pro-system as in (4.4.4) representing $X_{\infty}^{\infty}$. So $X_{\infty}^{\infty}=\lim _{\longrightarrow} X_{\infty}^{\alpha}=\lim _{\longrightarrow} \tilde{X}_{\infty}^{\tilde{\alpha}}$. The second equality (of ind-objects) means that each $X_{\infty}^{\alpha}$ is included into some $\tilde{X}_{\infty}^{\tilde{\alpha}}$ as a closed subset and vice versa. This means that $\mathbf{D}_{X_{\infty}^{\alpha} / S}$ is identified with a full subcategory in some $\mathbf{D}_{\tilde{X}_{\infty}^{\tilde{\alpha}} / S}$ and vice versa. Therefore their 2-limits are identified.

(4.4.6) Remark. Although we have defined $\mathbf{D}_{X_{\infty}^{\infty} / S}$ as an abstract category, it is impossible, in general, to view its objects as sheaves in a more conventional sense. For example, it is impossible to associate to an object of $\mathbf{D}_{X_{\infty}^{\infty} / S}$ its direct image onto $S$. Indeed, assume that $S=\operatorname{Spec}(k)$ for simplicity. If such an object $\mathcal{M}$ is represented by some $\mathcal{M}_{\beta}^{\alpha} \in \mathbf{D}_{X_{\beta}^{\alpha}}$, then the spaces of global sections of the coherent sheaves

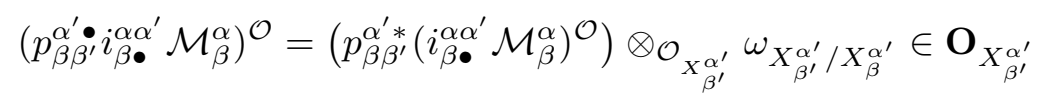

do not form an inductive system because of the twist by the relative canonical class. For a compact smooth pro-scheme it is possible to get around this problem by untwisting by the absolute canonical classes of the terms of the projective system, see (4.2.7). To achieve the same effect in the ind-pro-case one would need to make sense of the (absolute) canonical class of the ind-scheme $X_{\beta}^{\infty}$, i.e. of the determinant of the (possibly infinite-dimensional) vector bundle $\hat{\Theta}_{X_{\beta}^{\infty}}$. The impossibility of doing this ("the determinantal anomaly") is precisely the reason why there is no natural space $\Gamma\left(X_{\infty}^{\infty}, \mathcal{M}\right)$.

(4.4.7) Example. (a) If $X$ is a smooth affine variety admitting an étale map to $\mathbb{A}^{d}$, then the ind-scheme $\mathcal{L}(X)$ is locally compact and smooth. Thus, the category $\mathbf{D}_{\mathcal{L}(X)}$ is well-defined. If $X$ is no longer affine it admits a covering by affine open sets $U_{\alpha}$ admitting an étale map to $\mathbb{A}^{d}$. Then, an object of $\mathbf{D}_{\mathcal{L}(X)}$ is a sheaf on $\mathcal{L}(X)$ whose restriction to $\mathcal{L}\left(U_{\alpha}\right)$ is an object of $\mathbf{D}_{\mathcal{L}\left(U_{\alpha}\right)}$. 
(b) If $X$ is smooth then $\mathcal{L}(X)_{C^{I}} \rightarrow C^{I}$ is a locally compact and smooth indscheme over $C^{I}$.

(c) If $X$ is a smooth affine variety, then the ind-scheme $\tilde{\mathcal{L}}(X)$ is locally compact and formally smooth. But we do not know if it is smooth in the sense of (4.4.4). 


\section{De Rham Complexes on InD-SChemes}

As in $\S 4$, let $S$ be a $k$-scheme of finite type.

(5.1) Reminder on the De Rham complexes. Let $q: X \rightarrow S$ be a smooth $S$-scheme of finite type and $\mathcal{M} \in \mathbf{D}_{X / S}$ be a right $\mathcal{D}_{X / S}$-module. Its de Rham complex $\mathcal{D} \mathcal{R}(\mathcal{M})$ is given by

$$
\mathcal{D} \mathcal{R}^{i}(\mathcal{M})=\mathcal{H o m}_{\mathcal{O}_{X}}\left(\Omega_{X / S}^{-i}, \mathcal{M}\right)=\mathcal{M} \otimes_{\mathcal{O}_{X}} \bigwedge^{-i} \Theta_{X / S}, \quad i \leq 0 .
$$

If $\left(x_{j}\right)$ is a relative étale coordinate system on an open part of $X$, then the differential is given by the formula $d=\sum \partial_{x_{j}} \otimes d x_{j}$ where $d x_{j} \in \Omega_{X}^{1}$ is considered as the contraction operator $\bigwedge^{-i} \Theta_{X} \rightarrow \bigwedge^{-i-1} \Theta_{X}$. We denote by $D R(\mathcal{M})=q_{*}(\mathcal{D R}(\mathcal{M}))$ the complex of direct images.

Let $i: X \rightarrow Y$ be a closed embedding of smooth $S$-schemes and $\mathcal{M} \in \mathbf{D}_{X / S}$. The embedding (4.1.3) induces an embedding of the de Rham complexes $\mathcal{D} \mathcal{R}(\mathcal{M}) \hookrightarrow$ $i^{*} \mathcal{D} \mathcal{R}\left(i_{\bullet} \mathcal{M}\right)$ and therefore an embedding of the complexes of direct images

$$
D R(\mathcal{M}) \hookrightarrow D R\left(i_{\bullet}(\mathcal{M})\right)
$$

Let $p: X \rightarrow Y$ be a smooth morphism of smooth $S$-schemes of relative dimension $d$ and $\mathcal{M} \in \mathbf{D}_{Y / S}$. Let $q: X \rightarrow S, r: Y \rightarrow S$ be the structure maps. The embedding (4.1.2) induces an embedding of de Rham complexes which now involves a shift in the degrees:

$$
p^{*} \mathcal{D} \mathcal{R}(\mathcal{M}) \hookrightarrow \mathcal{D} \mathcal{R}\left(p^{\bullet}(\mathcal{M})\right)[d]
$$

It is induced by the map

$$
p^{*} \bigwedge^{i} \Theta_{Y / S} \rightarrow \omega_{X / Y} \otimes_{\mathcal{O}_{X}} \bigwedge^{i+d} \Theta_{X / S}
$$

In particular, we get an embedding of the complexes of direct images

$$
D R(\mathcal{M}) \hookrightarrow D R\left(p^{\bullet} \mathcal{M}\right)[d] .
$$

Note that without passing to the de Rham complexes there is no embedding of $r_{*} \mathcal{M}$ into $q_{*} p^{\bullet} \mathcal{M}$. The map (5.1.3) can be seen as a $\mathcal{D}$-module manifestation of the fact that "fermions cancel the determinantal anomaly".

(5.1.4) Example. Take $S=\operatorname{Spec}(k)$. Let $Y=\mathbb{A}^{d}$ with coordinates $a_{1}, \ldots, a_{d}$ and $i: X \hookrightarrow Y$ be the embedding of the affine subspace $\left\{a_{1}=\ldots=a_{l}=0\right\}, l \leq d$. The algebra $\Gamma\left(Y, \mathcal{D}_{Y}\right)$ is just the Heisenberg (Weyl) algebra $D_{Y}$ generated by $a_{1}, \ldots, a_{d}$ and $a_{1}^{*}, \ldots, a_{d}^{*}$ subject to the relations

$$
\left[a_{m}, a_{n}\right]=\left[a_{m}^{*}, a_{n}\right]=0, \quad\left[a_{m}^{*}, a_{n}\right]=\delta_{m n} .
$$


The space of global sections $\Gamma\left(Y, i_{\bullet} \omega_{X}\right)$ is the right $D_{Y}$-module

$$
\omega_{X Y}=D_{Y} /\left(a_{m}^{*}, a_{n} ; n \leq l<m\right) D_{Y} .
$$

Let also $C_{Y}$ be the Clifford algebra generated by odd elements $b_{1}, \ldots, b_{d}, b_{1}^{*}, \ldots, b_{d}^{*}$ subject to the relations

$$
\left[b_{m}, b_{n}\right]_{+}=\left[b_{m}^{*}, b_{n}^{*}\right]_{+}=0, \quad\left[b_{m}^{*}, b_{n}\right]_{+}=\delta_{m n} .
$$

Denote $C D_{Y}=C_{Y} \otimes_{k} D_{Y}$ the tensor product algebra. Then the global de Rham complex of $i_{\bullet} \omega_{X}$ is identified with the right $C D_{Y}$-module

$$
D R_{X Y}=C D_{Y} /\left(a_{m}^{*}, a_{n}, b_{p} ; n \leq l<m, p=1, \ldots, d\right) C D_{Y} .
$$

(5.2) De Rham complexes for ind-schemes. Let $X^{\infty}=\lim _{\alpha \in A} X^{\alpha}$ be a formally smooth discrete ind-S-scheme with structure maps $q, q^{\alpha}$. Denote by $i^{\alpha}$ : $X^{\alpha} \hookrightarrow X^{\infty}$ the canonical embedding. The considerations of (5.1) generalize easily to give the global de Rham complex of any $\mathcal{M} \in \mathbf{D}_{X^{\infty} / S}$. Explicitly, let $\mathcal{M}$ have the form $i_{\alpha} \cdot \mathcal{M}^{\alpha}$. Then the $i$ th term of its de Rham complex is

$$
D R^{i}(\mathcal{M})=\mathcal{M}^{\mathcal{O}} \otimes \bigwedge^{-i} \hat{\Theta}_{X \infty / S}=\lim _{\alpha^{\prime} \geq \alpha} q_{*}^{\alpha^{\prime}} \mathcal{H o m}\left(\Omega_{X^{\alpha^{\prime}} / S}^{-i},\left(i_{\bullet}^{\alpha \alpha^{\prime}} \mathcal{M}^{\alpha}\right)^{\mathcal{O}}\right)
$$

Here $\mathcal{M}^{\mathcal{O}}$ is defined in (4.3.6).

(5.2.2) Proposition. Let $p^{\infty}: X^{\infty} \rightarrow Y^{\infty}$ be a morphism of formally smooth discrete ind-S-schemes which is smooth of relative dimension $d$. Then for any $\mathcal{M} \in \mathbf{D}_{Y^{\infty} / S}$ we have an embedding of the shifted de Rham complexes $D R(\mathcal{M}) \hookrightarrow$

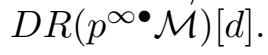

Proof: The conditions on $p^{\infty}$ in the proposition are equivalent to the following: we can represent $X^{\infty}=\lim _{\alpha \in A} X^{\alpha}, Y^{\infty}=\lim _{\alpha \in A} Y^{\alpha}$ with the same filtering poset $A$, and we can represent $p^{\infty}$ by a morphism of inductive systems $\left(p^{\alpha}: X^{\alpha} \rightarrow Y^{\alpha}\right)$ of $S$-schemes such that for each $\alpha \leq \beta$ the arising commutative square

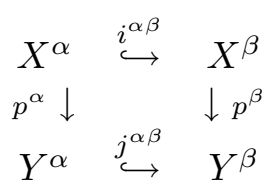

is Cartesian, and each $p^{\alpha}$ is smooth of relative dimension $d$. Then $p^{\infty}$ is formally smooth. By [BD2, Lemma 7.12.23] we have an exact sequence

$$
0 \rightarrow \hat{\Theta}_{X^{\infty} / Y^{\infty}} \rightarrow \hat{\Theta}_{X^{\infty} / S} \rightarrow p^{\infty *} \hat{\Theta}_{Y^{\infty} / S} \rightarrow 0
$$


Moreover

$$
\left(i^{\beta}\right)^{*}\left(\hat{\Theta}_{X^{\infty} / Y^{\infty}}\right)=\Theta_{X^{\beta} / Y^{\beta}} .
$$

Let $\mathcal{M}$ have the form $j_{\alpha} \cdot \mathcal{M}^{\alpha}$, where $j^{\alpha}: Y^{\alpha} \hookrightarrow Y^{\infty}$ is the canonical embedding.

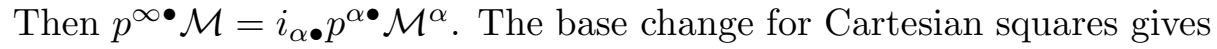

$$
\begin{aligned}
D R^{i}\left(p^{\infty} \mathcal{M}\right)[d] & =\lim _{\beta \geq \alpha} q_{*}^{\beta}\left(\left(p^{\beta \bullet} j_{\bullet}^{\alpha \beta} \mathcal{M}^{\alpha}\right)^{\mathcal{O}} \otimes_{\mathcal{O}_{X \beta} \beta} i^{\beta *} \bigwedge^{-i-d} \hat{\Theta}_{X^{\infty} / S}\right) \\
& =\lim _{\beta \geq \alpha} q_{*}^{\beta}\left(p^{\beta *}\left(j_{\bullet}^{\alpha \beta} \mathcal{M}^{\alpha}\right)^{\mathcal{O}} \otimes_{\mathcal{O}_{X^{\beta}}} \omega_{X^{\beta} / Y^{\beta}} \otimes_{\mathcal{O}_{X} \beta} i^{\beta *} \bigwedge^{-i-d} \hat{\Theta}_{X^{\infty} / S}\right),
\end{aligned}
$$

see Lemma 4.1.4. Let $r: Y^{\infty} \rightarrow S, r^{\alpha}: Y^{\alpha} \rightarrow S$ be the structure maps. By (5.2.3), (5.2.4) there is an embedding

$$
p^{\beta *} j^{\beta *} \bigwedge^{-i} \hat{\Theta}_{Y^{\infty} / S}=i^{\beta *} p^{\infty *} \bigwedge^{-i} \hat{\Theta}_{Y^{\infty} / S} \rightarrow \omega_{X^{\beta} / Y^{\beta}} \otimes_{\mathcal{O}_{X} \beta} i^{\beta *} \bigwedge^{-i-d} \hat{\Theta}_{X^{\infty} / S}
$$

Hence there is an embedding

$$
\begin{gathered}
D R^{i}(\mathcal{M})=\lim _{\beta \geq \alpha} r_{*}^{\beta}\left(\left(j_{\bullet}^{\alpha \beta} \mathcal{M}^{\alpha}\right)^{\mathcal{O}} \otimes_{\mathcal{O}_{Y} \beta} j^{\beta *} \bigwedge^{-i} \hat{\Theta}_{Y^{\infty} / S}\right) \rightarrow \\
\rightarrow \lim _{\beta \geq \alpha} q_{*}^{\beta}\left(p^{\beta *}\left(\left(j_{\bullet}^{\alpha \beta} \mathcal{M}^{\alpha}\right)^{\mathcal{O}} \otimes_{\mathcal{O}_{Y \beta}} j^{\beta *} \bigwedge^{-i} \hat{\Theta}_{Y^{\infty} / S}\right)\right) \rightarrow D R^{i}\left(p^{\infty} \mathcal{M}\right)[d] .
\end{gathered}
$$

We are done.

(5.3) De Rham complexes for ind-pro-schemes. Let $X_{\infty}^{\infty}$ be a locally compact smooth ind-S-scheme and $\mathcal{M}$ be an object of $\mathbf{D}_{X_{\infty}^{\infty} / S}$. We fix an ind-pro-system $\left(X_{\beta}^{\alpha}\right)$ for $X$ as in (4.4.1), (4.4.4). We have then the formally smooth discrete indschemes $X_{\beta}^{\infty}$ and projections $p_{\beta}: X_{\infty}^{\infty} \rightarrow X_{\beta}^{\infty}$. We also have schemes $X_{\infty}^{\alpha}$ and embeddings $i^{\alpha}: X_{\infty}^{\alpha} \rightarrow X_{\infty}^{\infty}$. Let also $i_{\beta}^{\alpha}: X_{\beta}^{\alpha} \rightarrow X_{\beta}^{\infty}, p_{\beta}^{\alpha}: X_{\infty}^{\alpha} \rightarrow X_{\beta}^{\alpha}$ be the natural embeddings and projections. The category $\mathbf{D}_{X_{\infty}^{\infty} / S}$ being the double direct limit of $\mathbf{D}_{X_{\beta}^{\alpha} / S}$, we can think of $\mathcal{M}$ as being of the form $p_{\beta}^{\bullet} i_{\beta}^{\alpha} \mathcal{M}_{\beta}^{\alpha}=i_{\bullet}^{\alpha} p_{\beta}^{\alpha \bullet} \mathcal{M}_{\beta}^{\alpha}$ for some $\mathcal{M}_{\beta}^{\alpha} \in \mathbf{D}_{X_{\beta}^{\alpha} / S}$. Recall that $d_{\beta \beta^{\prime}}$ denotes the relative dimension of the smooth morphism $p_{\beta \beta^{\prime}}^{\alpha}: X_{\beta^{\prime}}^{\alpha} \rightarrow X_{\beta}^{\alpha}, \beta \leq \beta^{\prime}$. We choose numbers $d_{\beta}, \beta \in B$, such that $d_{\beta \beta^{\prime}}=d_{\beta}-d_{\beta^{\prime}}$ (this can be done uniquely up to an overall constant). Set $\mathcal{M}_{\beta}=i_{\beta}^{\alpha} \mathcal{M}_{\beta}^{\alpha} \in \mathbf{D}_{X_{\beta}^{\infty} / S}$. Proposition 5.2.2 implies then that the shifted global de Rham complexes $D R\left(p_{\beta \beta^{\prime}}^{\bullet} \mathcal{M}_{\beta}\right)\left[d_{\beta^{\prime}}\right]$ form an inductive system of complexes of vector spaces and we define the de Rham complex of $\mathcal{M}$ to be

$$
D R(\mathcal{M})=\lim _{\beta^{\prime} \geq \beta} D R\left(p_{\beta \beta^{\prime}}^{\bullet} \mathcal{M}_{\beta}\right)\left[d_{\beta^{\prime}}\right]
$$

Explicitely, by (5.2.1) we have

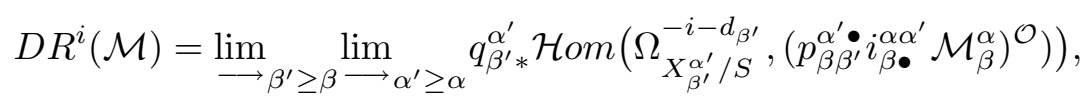

where $q_{\beta^{\prime}}^{\alpha^{\prime}}: X_{\beta^{\prime}}^{\alpha^{\prime}} \rightarrow S$ is the structure morphism. 
(5.3.3) Proposition. $D R^{i}(\mathcal{M})$ depends only on $X_{\infty}^{\infty}$ and $\mathcal{M}$ as an object of $\mathbf{D}_{X_{\infty}^{\infty} / S}$, but not on the choice of a system $\left(X_{\beta}^{\alpha}\right)$.

Proof: Interchanging the two inductive limits and using base change for Cartesian squares in the diagram $\left(X_{\beta}^{\alpha}\right)$, we can write

$$
\left.D R^{i}(\mathcal{M})=\lim _{\longrightarrow \alpha^{\prime} \geq \alpha} \lim _{\beta^{\prime} \geq \beta} q_{\beta^{\prime} *}^{\alpha^{\prime}} \mathcal{H o m}\left(\Omega_{X_{\beta^{\prime}}^{\alpha^{\prime} / S}}^{-i-d_{\beta^{\prime}}},\left(i_{\beta^{\prime} \bullet}^{\alpha \alpha^{\prime}} p_{\beta \beta^{\prime}}^{\alpha \bullet} \mathcal{M}_{\beta}^{\alpha}\right)^{\mathcal{O}}\right)\right)
$$

For any $\alpha^{\prime} \geq \alpha$ the limit over $\beta^{\prime}$ depends only on the scheme $X_{\infty}^{\alpha^{\prime}}$ and the object

$$
\mathcal{M}^{\alpha^{\prime}}=p_{\beta}^{\alpha^{\prime} \bullet} i_{\beta}^{\alpha \alpha^{\prime}} \mathcal{M}_{\beta}^{\alpha} \in \mathbf{D}_{X_{\infty}^{\alpha^{\prime}} / S}
$$

Therefore the limit over $\alpha^{\prime} \geq \alpha$ of the limits above depends only on the ind-object $\stackrel{\text { "lim }}{\longrightarrow \alpha^{\prime} \geq \alpha}$ " $X_{\infty}^{\alpha^{\prime}}$ (which is $\left.X_{\infty}^{\infty}\right)$ and the object

$$
i_{\alpha^{\prime} \bullet} \mathcal{M}^{\alpha^{\prime}} \in 2 \underset{\lim _{\alpha^{\prime} \geq \alpha}}{ } \mathbf{D}_{X_{\infty}^{\alpha^{\prime}} / S}=\mathbf{D}_{X_{\infty}^{\infty} / S}
$$

which is $\mathcal{M}$.

(5.4) The de Rham complexes on $\mathcal{L}(X)$. We now specialize to the particular case $X_{\infty}^{\infty}=\mathcal{L}(X)$ where $X$ is a smooth affine algebraic variety over $k$ admitting an étale map $\phi$ to $\mathbb{A}^{d}$. In this case $A=\mathbb{E}, B=\mathbb{N}$ with the terms of the indpro-systems being $\mathcal{L}_{n}^{\varepsilon}(\phi)$, see (2.6.7). We take $S=\operatorname{Spec}(k)$. Given an object $\mathcal{M} \in \mathbf{D}_{\mathcal{L}(X)}$ we associate to it its de Rham complex $D R(\mathcal{M})$ as in (5.3). Note that it is independent on the choice of an étale map to $\mathbb{A}^{d}$ because two such maps lead to isomorphic ind-pro-objects in the category $\mathbf{S} \mathbf{c h}^{\mathrm{ft}}$, see Proposition 2.7.1.(c).

Let now $X$ be an arbitrary smooth algebraic variety over $k$ and $\mathcal{M}$ be an object of $\mathbf{D}_{\mathcal{L}(X)}$. By covering $X$ with affine open $U$ admitting étale maps to $\mathbb{A}^{d}$, we get a complex of sheaves $U \mapsto D R\left(\left.\mathcal{M}\right|_{\mathcal{L}(U)}\right)$ which we denote $\mathcal{D} \mathcal{R}(\mathcal{M})$. Recall that we have the diagram

$$
X \stackrel{p}{\longleftarrow} \mathcal{L}^{0}(X) \stackrel{i}{\hookrightarrow} \mathcal{L}(X) .
$$

Thus, every right $\mathcal{D}_{X}$-module $\mathcal{N}$ gives an object $i_{\bullet} p^{\bullet} \mathcal{N}$ of $\mathbf{D}_{\mathcal{L}(X)}$. We write $\mathcal{C D} \mathcal{R}(\mathcal{N})$ for $\mathcal{D} \mathcal{R}\left(i_{\bullet} p^{\bullet} \mathcal{N}\right)$ and call it the chiral de Rham complex of $\mathcal{N}$. In particular, we write $\mathcal{C D} \mathcal{R}_{X}$ for $\mathcal{D} \mathcal{R}\left(i_{\bullet} p \omega^{\bullet}\right)$. More generally, denoting $p_{n}: \mathcal{L}^{0}(X) \rightarrow \mathcal{L}_{n}^{0}(X)$ the projection, we can start with any right $\mathcal{D}$-module $\mathcal{N}$ on the algebraic variety $\mathcal{L}_{n}^{0}(X)$ : then $i_{\bullet} p_{n}^{\bullet} \mathcal{N}$ is an object of $\mathbf{D}_{\mathcal{L}(X)}$ and we can form its de Rham complex. It is a complex of sheaves on $X$.

(5.4.2) Example. Let $X=\mathbb{A}^{1}$. Then the complex of global sections of $\mathcal{C D} \mathcal{R}_{X}$, i.e., the complex $D R\left(i_{\bullet} p^{\bullet} \omega_{\mathbb{A}^{1}}\right)$ can be found explicitly as follows. 
Let $V$ be the topological $k$-vector space $k((t))$ and $V^{*}$ be its topological dual (over $k$ ). Denote by $=\langle l, v\rangle$ the canonical pairing of $l \in V^{*}$ and $v \in V$.

Then $V^{*}$ can be identified with $k((t)) d t$, the space of 1 -forms, the pairing between $V$ and $V^{*}$ being $(f, \omega) \rightarrow \operatorname{res}(f \cdot \omega)$. Let $D$ be the Heisenberg algebra generated by $V^{*}$ and $V$ with $[l, v]=\langle l, v\rangle$ and $C$ be the Clifford algebra generated by $V^{*}, V$ with $[l, v]_{+}=\langle l, v\rangle$. Denote $C D=C \otimes_{k} D$. This is a certain completion of the algebra $\tilde{C D}$ generated by symbols $a_{n}, b_{n}, a_{n}^{*}, b_{n}^{*}$ for $n \in \mathbb{Z}$ subject to the relations

$$
\begin{gathered}
{\left[a_{m}, a_{n}\right]=\left[a_{m}^{*}, a_{n}^{*}\right]=0, \quad\left[a_{m}^{*}, a_{n}\right]=\delta_{m,-n},} \\
{\left[b_{m}, b_{n}\right]_{+}=\left[b_{m}^{*}, b_{n}^{*}\right]_{+}=0, \quad\left[b_{m}^{*}, b_{n}\right]_{+}=\delta_{m,-n},} \\
{\left[a_{m}, b_{n}\right]=\left[a_{m}^{*}, b_{n}\right]=\left[a_{m}, b_{n}^{*}\right]=\left[a_{m}^{*}, b_{n}^{*}\right]=0 .}
\end{gathered}
$$

More precisely, we write a generic element of $V$ as $\sum_{m} a_{m} t^{m}$, so $a_{m}, a_{m}^{*}$ are elements of $D$. Similarly, writing a generic element of $V^{*}$ as $\sum_{m} b_{m} t^{m-1} d t$ we view $b_{m}, b_{m}^{*}$ as elements of $C$. Let $\tilde{V}=k\left[t, t^{-1}\right], \tilde{V}^{*}=k\left[t, t^{-1}\right] d t$. Then $\tilde{C D}=\tilde{C} \otimes_{k} \tilde{D}$, where $\tilde{C}$ is the Clifford algebra generated by $V^{*}, V$ and $\tilde{D}$ is the Heisenberg algebra generated by $\tilde{V}, \tilde{V}^{*}$. Let $\tilde{C D}{ }^{+} \subset \tilde{C D}$ be the right ideal generated by linear combinations of $a_{n}, b_{n}, a_{n+1}^{*}, b_{n+1}^{*}$ with $n \geq 0$, and $C D^{+} \subset C D$ be the ideal generated by possibly infinite linear combinations with the above property. We denote $V a c=C D / C D^{+}$ and $\tilde{V a c}=\tilde{C D} / \tilde{C D}{ }^{+}$the corresponding vacuum modules.

(5.4.3) Proposition. (a) The natural morphism $\tilde{V a c} \rightarrow$ Vac is an isomorphism.

(b) The de Rham complex $\operatorname{DR}\left(i_{\bullet} p^{\bullet} \omega_{\mathbb{A}^{1}}\right)$ is identified (as a vector space) with Vac.

Remark. Although this description is similar to (5.1.4), there is a difference : here the ideal is generated by $b_{n+1}^{*}, b_{n}$ for $n<0$ while in (5.1.4) all the $b_{n}$ are in the ideal. This is because in our present situation we are dealing with a semiinfinite de Rham complex obtained as an inductive limit of usual de Rham complexes with respect to maps shifting the degrees.

Proof: (a) The quotient $k((t)) / k[[t]]$ is identified with $k\left[t, t^{-1}\right] / k[t]$. The ideal $C D^{+}$ includes the Taylor series part of $V, V^{*} \subset D$ and $V, V^{*} \subset C$. So $C D / C D^{+}$is identified with $\tilde{C D} / \tilde{C D}^{+}$.

(b) To simplify, we write $\mathcal{L}=\mathcal{L}\left(\mathbb{A}^{1}\right)$, etc. We have

$$
\mathcal{L}_{M}^{\varepsilon}=\operatorname{Spec}\left(k\left[a_{l} ;-N_{\varepsilon} \leq l \leq M\right] /\left(a_{l}^{1+\varepsilon_{l}}\right)\right),
$$

where, for each $\varepsilon \in \mathbb{E}$, we set $N_{\varepsilon}=\max \left\{l ; \varepsilon_{-l} \neq 0\right\}$. Hence

$$
\mathcal{L}_{M}=\lim _{N} \operatorname{Spf}\left(k\left[a_{l} ; 0 \leq l \leq M\right]\left[\left[a_{l} ;-N \leq l<0\right]\right]\right) .
$$


For any $M \in \mathbb{N}, N \in \mathbb{N} \cup\{\infty\}$, we put

$$
Y_{M}^{N}=\operatorname{Spec}\left(k\left[a_{l} ;-N \leq l \leq M\right]\right) .
$$

Then $\mathcal{L}_{M}$ is just the limit, over $N>0$, of the formal completions of $Y_{M}^{N}$ along $Y_{M}^{0}$. Since the de Rham complex with coefficients in the $\mathcal{D}$-module of distributions along a subvariety depends only on the completion along this subvariety, we can write

$$
D R\left(i \bullet p^{\bullet} \omega_{\mathbb{A}^{1}}\right)=\lim _{M, N} D R\left(i_{M, N, \bullet} \omega_{Y_{M}^{0}}\right)[M],
$$

where $i_{M, N}: Y_{M}^{0} \hookrightarrow Y_{M}^{N}$ is the embedding. Note that $i_{M, N}$ is just the embedding of an affine subspace, so we are in the situation of Example 5.1.4.

Let $D_{M}^{N}$ be the subalgebra in $C D$ generated by $a_{n},-N \leq n \leq M$, and $a_{n}^{*}$, $-M \leq n \leq N$. It is identified with the algebra of polynomial differential operators on functions of $a_{-N}, \ldots, a_{M}$, with $a_{-i}^{*}$ corresponding to $\partial / \partial a_{i}$. Similarly let $C_{M}^{N}$ be generated by $b_{n},-N \leq n \leq M$, and $b_{n}^{*},-M \leq n \leq N$. Denote $C D_{M}^{N}=$ $C_{M}^{N} \otimes_{k} D_{M}^{N}$. We see therefore that the $(M, N)$ th term of the inductive system in (5.4.4) is identified with

$$
\operatorname{Vac}_{M}^{N}:=C D_{M}^{N} /\left(a_{i}, i>0 ; a_{i}^{*}, i \geq 0 ; b_{i}^{*}\right) C D_{M}^{N}
$$

Denote by $1_{M}^{N}$ the generator of this module. Then for $N \leq N^{\prime}$ and any $M$ the embedding takes $1_{M}^{N}$ to $1_{M}^{N^{\prime}}$, while for $M \leq M^{\prime}$ and any $N$ it takes $1_{M}^{N}$ into $1_{M^{\prime}}^{N}, b_{M+1} \ldots b_{M^{\prime}}$. From the normal form of elements it is clear that $\tilde{C D}=$ $\lim _{M, N} C D_{M}^{N}, \tilde{V a c}=\lim _{M, N} V a c_{M}^{N}$, and we are done since $\tilde{V a c}=V a c$. 


\section{Identification of the Chiral De Rham Complex}

In this section we construct, in a geometric way, the structure of a vertex algebra on the chiral de Rham complex $\mathcal{C D} \mathcal{R}_{X}$ and compare it with the construction of [MSV].

(6.1) Factorization algebras, and De Rham complexes on $\mathcal{L}(X)_{C^{I}}$. Let $C$ be a smooth curve, as before. For any non-empty finite set $I$ we set $U^{(I)}=U^{(I / I)}$, $\Delta^{(I)}=\Delta^{(I /\{1\})}$ and $j^{(I)}=j^{(I / I)}$, see (3.2). Hereafter we write $U, \Delta, j$ instead of $U^{(I)}, \Delta^{(I)}, j^{(I)}$ if $I$ has cardinal 2. We will need the following notation : for any (possibly empty) $I$ let $\bar{I}=I \sqcup \varnothing$ be the corresponding pointed set. For any surjection $I \rightarrow J$ we denote $\bar{I} \rightarrow \bar{J}$ the surjection equal to $I \rightarrow J$ on $I$ and taking $\varnothing$ to $\varnothing$. Let us recall the definition of a factorization algebra, see [BD1, §3.4].

(6.1.1) Definition. (a) Let $\mathcal{E}$ be a quasi-coherent sheaf on $C$. A structure of a factorization algebra on $\mathcal{E}$ is a collection of quasi-coherent $\mathcal{O}_{C^{I}}$-modules $\mathcal{E}_{I}$ for each non-empty finite set $I$, such that $\mathcal{E}_{I}$ is flat along the diagonal strata, $\mathcal{E}_{\{1\}}=\mathcal{E}$, and

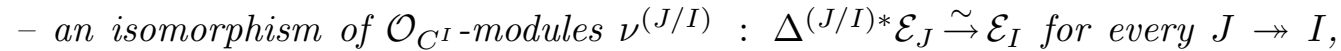
compatible with the compositions of $J \rightarrow I$,

- an isomorphism of $\mathcal{O}_{U^{(J / I)}}$-modules

$$
\varkappa^{(J / I)}: j^{(J / I) *}\left(\bigotimes_{I} \mathcal{E}_{J_{i}}\right) \stackrel{\sim}{\rightarrow} j^{(J / I) *} \mathcal{E}_{J}
$$

for every $J \rightarrow I$, compatible with the compositions of $J \rightarrow I$, and compatible with $\nu$,

- a global section $1_{\mathcal{E}} \in \mathrm{H}^{0}(C, \mathcal{E})$ such that for every $f \in \mathcal{E}$ one has $1_{\mathcal{E}} \otimes f \in$ $\mathcal{E}_{\{1,2\}} \subset j_{*} j^{*}(\mathcal{E} \otimes \mathcal{E})$ and $\Delta^{*}\left(1_{\mathcal{E}} \otimes f\right)=f$.

(b) A module over $\mathcal{E}$ is a quasi-coherent sheaf $\mathcal{M}$ on $C$ with a collection of quasicoherent $\mathcal{O}_{C^{\bar{I}} \text {-modules }} \mathcal{M}_{\bar{I}}$ for each non-empty finite set $I$, such that $\mathcal{M}_{\bar{I}}$ is flat along the diagonal strata, $\mathcal{M}_{\{\odot\}}=\mathcal{M}$, and

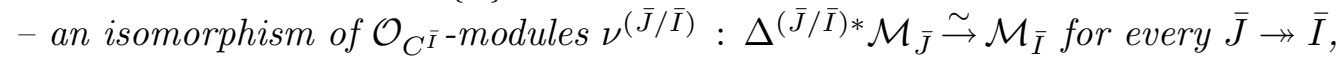
compatible with the compositions of $\bar{J} \rightarrow \bar{I}$,

- an isomorphism of $\mathcal{O}_{U^{(\bar{J} / \bar{I})}}$-modules

$$
\varkappa^{(\bar{J} / \bar{I})}: j^{(\bar{J} / \bar{I}) *}\left(\left(\bigotimes_{I} \mathcal{E}_{J_{i}}\right) \otimes \mathcal{M}_{\bar{J}_{\bigodot}}\right) \stackrel{\sim}{\rightarrow} j^{(\bar{J} / \bar{I}) *} \mathcal{M}_{\bar{J}}
$$

for every $\bar{J} \rightarrow \bar{I}$, compatible with the compositions of $\bar{J} \rightarrow \bar{I}$, and compatible with $\nu$, such that

- for any $f \in \mathcal{M}$ one has $1_{\mathcal{E}} \otimes f \in \mathcal{M}_{\{1, \mathcal{O}\}} \subset j_{*} j^{*}(\mathcal{E} \otimes \mathcal{M})$ and $\Delta^{*}\left(1_{\mathcal{E}} \otimes f\right)=f$.

We have the following immediate global counterpart of (5.4). 
(6.1.2) Proposition. For any right $\mathcal{D}_{X}$-module $\mathcal{M}$ there is a unique complex

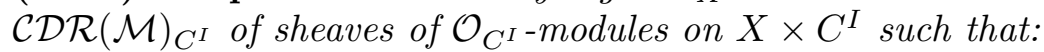

(a) the fiber of $\mathcal{C D} \mathcal{R}(\mathcal{M})_{C}$ at a point of the curve $C$ is isomorphic to the complex $\mathcal{C D} \mathcal{R}(\mathcal{M})$

(b) the collection $\left(\mathcal{C D} \mathcal{R}\left(\omega_{X}\right)_{C^{I}}\right)$ is a factorization algebra on the curve $C$. The collection $\left(\mathcal{C D} \mathcal{R}(\mathcal{M})_{C^{I}}\right)$ is a $\left(\mathcal{C D} \mathcal{R}\left(\omega_{X}\right)_{C^{I}}\right)$-module.

Proof: A choice of an element $i_{0} \in I$ defines a morphism of schemes $p_{i_{0}, I}$ : $\mathcal{L}^{0}(X)_{C^{I}} \rightarrow X$ as follows. Recall that $\mathcal{L}^{0}(X)_{C^{I}}$ represents the functor $\lambda_{X, C^{I}}^{0}$ which takes a scheme $S$ into the set of pairs $\left(f_{I}, \rho\right)$ where $f_{I}: S \rightarrow C^{I}$ is a morphism of schemes and $\rho$ is a morphism of $\widehat{\Gamma\left(f_{I}\right)}$, the formal neighborhood of $\Gamma\left(f_{I}\right) \subset S \times C$, into $X$. Now, restricting $\rho$ onto the graph of $f_{i_{0}}$, which is a subscheme in $\widehat{\Gamma\left(f_{I}\right)}$ isomorphic to $S$, we get a natural transformation from $\lambda_{X, C^{I}}^{0}$ into the functor represented by $X$, so a morphism $p_{i_{0}, I}$.

Denote by $\iota_{\bar{I}}: \mathcal{L}^{0}(X)_{C^{\bar{I}}} \rightarrow \mathcal{L}(X)_{C^{\bar{I}}}$ the embedding. For any $\mathcal{M} \in \mathbf{D}_{X}$, we form the object $\left(\iota_{\bar{I}}\right)_{\bullet}\left(p_{\odot, \bar{I}}\right)^{\bullet \mathcal{M}} \in \mathbf{D}_{\mathcal{L}(X)_{C \bar{I}} / C^{\bar{I}}}$. The general construction of (5.3), applied to the restrictions of $\left(\iota_{\bar{I}}\right) \bullet\left(p_{\odot, \bar{I}}\right) \bullet \mathcal{M}$ onto open subsets in $X \times C^{\bar{I}}$, gives then a complex of sheaves on $X \times C^{\bar{I}}$ which we denote $\mathcal{C D} \mathcal{R}(\mathcal{M})_{C^{\bar{I}}}$.

Notice that in the particular case where $\mathcal{M}=\omega_{X}$ the object $\left(p_{i_{0}, I}\right)^{\bullet} \mathcal{M}$ of the category $\mathbf{D}_{\mathcal{L}^{0}(X)_{C I} / C^{I}}$ is independent (up to a unique isomorphism) of the choice of $i_{0} \in I$. Indeed, objects of the latter category are, by definition, pairs $(n, \mathcal{N})$ where $\mathcal{N}$ is a right $\mathcal{D}$-module on $\mathcal{L}_{n}^{0}(X)_{C^{I}}$ and two such pairs $(n, \mathcal{N})$ and $\left(n^{\prime}, \mathcal{N}^{\prime}\right)$ are isomorphic, if the pullbacks of $\mathcal{N}$ and $\mathcal{N}^{\prime}$ to $\mathcal{L}_{m}^{0}(X)_{C^{I}}, m \geq n, n^{\prime}$, are isomorphic as right $\mathcal{D}$-modules. Since the pullback for right $\mathcal{D}$-modules is just the $\mathcal{O}$-module pullback tensored with the relative canonical class, $\left(p_{i_{0}, I}\right)^{\bullet} \omega_{X}$ is represented by $\left(n, \omega_{\mathcal{L}_{n}^{0}(X)_{C I}}\right)$ for any $n$, and thus is clearly independent on $i_{0}$.

Then, the general construction in (5.3) gives a complex of sheaves on $X \times C^{I}$, denoted by $\mathcal{C D} \mathcal{R}\left(\omega_{X}\right)_{C^{I}}$.

To prove Claim $(a)$ it is sufficient to observe that the fiber of $\mathcal{L}(X)_{C}$ at a point $0 \in C$ is isomorphic to $\mathcal{L}(X)$. Recall that $\mathcal{L}(X)_{C}$ represents the contravariant functor $\lambda_{X, C}: \mathbf{S c h} \rightarrow$ Sets such that $\lambda_{X, C}(S)$ is the set of pairs $(f, \rho)$ such that

$$
f \in \operatorname{Hom}_{\mathbf{S c h}}(S, C) \quad \text { and } \quad \rho \in \operatorname{Hom}_{\mathbf{L r s}}\left(\left(\Gamma(f), \mathcal{K}_{f}^{\sqrt{ }}\right), X\right)
$$

Thus the fiber at 0 represents the subfunctor

$$
S \mapsto\left\{(f, \rho) \in \lambda_{X, C}(S) \mid f(S)_{\mathrm{red}}=\{0\}\right\}
$$

Let $t$ be a local coordinate on $C$ centered at 0 . For any $f$ as above we have $\left(\Gamma(f), \mathcal{K}_{f}^{\sqrt{ }}\right)=\left(S, \mathcal{O}_{S}((t))^{\sqrt{ }}\right)$ and this proves $(\mathrm{a})$. Note that the isomorphism of 
$\mathcal{L}(X)$ and the fiber of $\mathcal{L}(X)_{C}$ at 0 is compatible with the ind-pro-systems in (2.7), (3.9).

(b) Both $\mathcal{L}^{0}(X)_{C^{I}}$ and $\mathcal{L}(X)_{C^{I}}$ form factorization monoids in the categories of ind-schemes. Since passing to the De Rham complex takes Cartesian products of (ind-)schemes to tensor products of vector spaces, we see that $\left(\mathcal{C D} \mathcal{R}\left(\omega_{X}\right)_{C^{I}}\right)$ form a factorization algebra.

Next, given any surjection $J \rightarrow I$ and the corresponding surjection $\bar{J} \rightarrow \bar{I}$, we have

$$
\prod_{\bar{i} \in \bar{I}} \mathcal{L}(X)_{C_{\bar{i}}^{\bar{J}}}=\mathcal{L}(X)_{C} \times \prod_{i \in I} \mathcal{L}(X)_{C^{J_{i}}}
$$

the first factor in the RHS corresponding to $\bar{i}=\varnothing$. Let us use the notation $\kappa$ for the factorization monoid structure of $\mathcal{L}(X)_{C^{I}}$ as in (3.2.1). Then, with respect to the identification (6.1.3), we have an isomorphism of $\mathcal{D}$-modules

$$
\left(\kappa^{(\bar{J} / \bar{I})}\right)^{\bullet}\left(\iota_{\bar{I}}\right) \bullet\left(p_{\odot, \bar{I}}\right)^{\bullet} \mathcal{M}=\left(p_{\odot,\{\odot\}}\right)^{\bullet} \mathcal{M} \otimes \bigotimes_{i \in I}\left(\iota_{I_{i}}\right) \bullet\left(p_{\odot, I_{i}}\right)^{\bullet} \omega_{X}
$$

over $U^{\bar{J} / \bar{I}} \rightarrow C^{\bar{I}}$. Using again the fact that passing to the De Rham complexes takes Cartesian products to tensor products, we conclude that $\left(\mathcal{C D} \mathcal{R}(\mathcal{M})_{C^{\bar{I}}}\right)$ is a factorization module over $\left(\mathcal{C D} \mathcal{R}\left(\omega_{X}\right)_{C^{I}}\right)$.

(6.2) Reminder on chiral and vertex algebras. Let us recall the basic facts on chiral and vertex algebras. See [BD1, §3], [K] and [FLM] for more details. Let $C$ be a smooth curve, as before. For any right $\mathcal{D}_{C}$-module $\mathcal{M}$ the projection formula yields an isomorphism of right $\mathcal{D}_{C^{2}}$-modules $\Delta_{\bullet} \Delta \bullet\left(\omega_{C} \otimes \mathcal{M}\right) \stackrel{\sim}{\rightarrow} \Delta_{.} \mathcal{M}$. Let

$$
\varepsilon_{\mathcal{M}}: j_{\bullet} j^{\bullet}\left(\omega_{C} \otimes \mathcal{M}\right) \rightarrow \Delta_{\bullet} \mathcal{M}
$$

be the composition of the projection $j_{\bullet} j^{\bullet}\left(\omega_{C} \otimes \mathcal{M}\right) \rightarrow j_{\bullet} j^{\bullet}\left(\omega_{C} \otimes \mathcal{M}\right) /\left(\omega_{C} \otimes \mathcal{M}\right)$ and of the isomorphism $\left(j_{\bullet} j^{\bullet}\left(\omega_{C} \otimes \mathcal{M}\right)\right) /\left(\omega_{C} \otimes \mathcal{M}\right) \stackrel{\sim}{\rightarrow} \Delta_{\bullet} \Delta^{\bullet}\left(\omega_{C} \otimes \mathcal{M}\right) \stackrel{\sim}{\rightarrow} \Delta_{\bullet} \mathcal{M}$.

(6.2.1) Definition. (a) A chiral algebra over $C$ is a right $\mathbb{Z} / 2 \mathbb{Z}$-graded $\mathcal{D}_{C}$-module $\mathcal{A}=\mathcal{A}^{0} \oplus \mathcal{A}^{1}$ with two even maps $\mu_{\mathcal{A}} \in \operatorname{Hom}_{\mathbf{D}_{C^{2}}}\left(j_{\bullet} j^{\bullet}(\mathcal{A} \otimes \mathcal{A}), \Delta_{\bullet} \mathcal{A}\right)$ and $1_{\mathcal{A}} \in$ $\operatorname{Hom}_{\mathbf{D}_{C}}\left(\omega_{C}, \mathcal{A}^{0}\right)$ such that

- the map $\mu_{\mathcal{A}}\left(1_{\mathcal{A}}, i d_{\mathcal{A}}\right)$ coincides with $\varepsilon_{\mathcal{A}}$,

- the map $\mu_{\mathcal{A}}$ is antisymmetric, and it satisfies the Jacobi identity.

(b) A module over a chiral algebra $\mathcal{A}$ over $C$ is a right $\mathbb{Z} / 2 \mathbb{Z}$-graded $\mathcal{D}_{C}$-module $\mathcal{M}_{C}$ with an even map $\mu_{\mathcal{M}} \in \operatorname{Hom}_{\mathbf{D}_{C^{2}}}\left(j_{\bullet} j^{\bullet}(\mathcal{A} \otimes \mathcal{M}), \Delta \cdot \mathcal{M}\right)$ such that

- the map $\mu_{\mathcal{M}}\left(1_{\mathcal{A}}, i d_{\mathcal{M}}\right)$ coincides with $\varepsilon_{\mathcal{M}}$,

- the map $\mu_{\mathcal{M}}$ is compatible with $\mu_{\mathcal{A}}$.

For any factorization algebra on $\mathcal{E}$, each sheaf $\mathcal{E}_{I}$ has a canonical left $\mathcal{D}_{C^{I}}$-module structure, compatible with the factorization structure, such that the section $1_{\mathcal{E}}$ is 
a horizontal, see [BD1, Proposition 3.4.8]. It is proved in [BD1, §3.4.9] that the right $\mathcal{D}_{C}$-module $\mathcal{E}^{r}:=\mathcal{E} \otimes_{\mathcal{O}_{C}} \omega_{C}$ is a chiral algebra over $C$. The map $\mu_{\mathcal{A}}$ is the composition of the chain of maps

$$
j \bullet j \cdot\left(\mathcal{E}^{r} \otimes \mathcal{E}^{r}\right)=j \bullet j \cdot \omega_{C^{2}} \otimes_{\mathcal{O}_{C^{2}}} \mathcal{E}_{\{1,2\}} \rightarrow \Delta_{\bullet} \omega_{C} \otimes_{\mathcal{O}_{C^{2}}} \mathcal{E}_{\{1,2\}}=\Delta_{\bullet} \mathcal{E}^{r}
$$

Here the 1-st equality is the 2-nd isomorphism in 6.1.1.(a), the second arrow is $\varepsilon_{\omega_{C}}$, and the last equality results from the 1-st isomorphism in 6.1.1.(a) and the projection formula for $\Delta$.

(6.2.2) Definition. (a) A vertex algebra is a $k$-supervector space $V$ with an even vector $1_{V} \in V$, an even endomorphism $\partial_{V} \in \operatorname{End}(V)$, and an even linear map $V \rightarrow \operatorname{End}(V)\left[\left[z, z^{-1}\right]\right], a \mapsto a(z)=\sum_{n} a_{n} z^{-n-1}$. These data satisfy the following axioms:

$-\partial_{V}\left(1_{V}\right)=0,1_{V}(z)=i d_{V}, a_{n}\left(1_{V}\right)=0$ if $n \geq 0, a_{-1}\left(1_{V}\right)=a$,

$-\left[\partial_{V}, a(z)\right]=\partial_{z} a(z)$,

- we have $(z-w)^{N}[a(z), b(w)]=0$ for $N>>0$.

We will also assume that for all elements $a, b \in V$ we have $a_{n}(b)=0$ for $n>>0$.

(b) A module over a vertex algebra $V$ is a $k$-supervector space $W$ with an even endomorphism $\partial^{W} \in \operatorname{End}(W)$, and an even linear map $V \rightarrow \operatorname{End}(W)\left[\left[z, z^{-1}\right]\right], a \mapsto$ $a^{W}(z)=\sum_{n} a_{n}^{W} z^{-n-1}$. These data satisfy the following axioms :

$-1_{V}^{W}(z)=i d_{W}$

$-\left[\partial^{W}, a^{W}(z)\right]=\partial_{z} a^{W}(z)=\left(\partial_{V} a\right)^{W}(z)$,

- (Borcherds identity)

$$
\begin{gathered}
z_{0}^{-1} \delta\left(\frac{z_{1}-z_{2}}{z_{0}}\right) a^{W}\left(z_{1}\right) b^{W}\left(z_{2}\right)-z_{0}^{-1} \delta\left(\frac{-z_{2}+z_{1}}{z_{0}}\right) b^{W}\left(z_{2}\right) a^{W}\left(z_{1}\right)= \\
=z_{2}^{-1} \delta\left(\frac{z_{1}-z_{0}}{z_{2}}\right)\left(a\left(z_{0}\right)(b)\right)^{W}\left(z_{2}\right)
\end{gathered}
$$

where

$$
z_{0}^{-1} \delta\left(\frac{z_{1}-z_{2}}{z_{0}}\right)=\sum_{m \in \mathbb{N}} \sum_{n \in \mathbb{Z}}(-1)^{m}\left(\begin{array}{c}
n \\
m
\end{array}\right) z_{0}^{-n-1} z_{1}^{n-m} z_{2}^{m}
$$

Assume that $C$ is the formal disk Spec $k[[t]]$. Let 0 be the closed point of $C$. Let $t_{1}=t \otimes 1, t_{2}=1 \otimes t$ be the coordinates on $C^{2}$. We have the following basic fact, due to Beilinson-Drinfeld (see [HL], [B] for details). Fix a vertex algebra $V$. The $k[[t]]$-module $V[[t]]$ has a unique structure of vertex algebra such that

$$
\partial_{V[[t]]}=\partial_{V}+\partial_{t}, \quad 1_{V[[t]]}=1_{V}, \quad\left(a t^{n}\right)(z)=(t+z)^{n} a(z),
$$

for any elements $a \in V, n \in \mathbb{Z}$. Let $\mathcal{A}_{V}$ be the sheaf on $C$ associated to the $k[[t]]$ module $V[[t]] \cdot d t$. The sheaf $\mathcal{A}_{V}$ has a unique structure of a chiral algebra over $C$ 
such that the field $\partial_{t}$ acts on $\mathcal{A}_{V}$ as the operator $\partial_{V[t]]}$, and such that the chiral product is induced by the map

$$
V \otimes V\left[\left[t_{1}, t_{2}\right]\right]\left[\left(t_{1}-t_{2}\right)^{-1}\right] \rightarrow V\left[\left[t_{1}, t_{2}\right]\right]\left[\left(t_{1}-t_{2}\right)^{-1}\right] / V\left[\left[t_{1}, t_{2}\right]\right]
$$

which takes the element $f\left(t_{1}, t_{2}\right) a \otimes b$, with $f\left(t_{1}, t_{2}\right) \in k\left(t_{1}-t_{2}\right)$ and $a, b \in V$, to the element $f\left(t_{1}, t_{2}\right) a\left(t_{1}-t_{2}\right)(b)+V\left[\left[t_{1}, t_{2}\right]\right]$. Similarly, if $W$ is a $V$-module then the $k[[t]]$-module $W[[t]]$ has a natural structure of a $V[[t]]$-module, and the corresponding sheaf $\mathcal{M}_{W}$ on $C$ has a natural structure of a $\mathcal{A}_{V}$-module. Conversely, we have the following.

(6.2.3) Lemma. Assume that $C$ is a smooth curve. Fix a point $0 \in C$ and a formal coordinate $t$ at 0 .

(a) Let $\mathcal{A}$ be a chiral algebra on $C$. Assume that $\mathcal{A}$ is a locally free $\mathcal{O}_{C}$-module. The fiber, $V$, of $\mathcal{A}$ at 0 has a unique structure of a vertex algebra such that the chiral algebra $\mathcal{A}_{V}$ is isomorphic to $\left.\mathcal{A}\right|_{\text {Spec } k[[t]]}$.

(b) Let $\mathcal{M}$ be a module over a chiral algebra $\mathcal{A}$ on $C$. Assume that $\mathcal{A}, \mathcal{M}$ are locally free $\mathcal{O}_{C}$-modules. Let $V, W$ be the fibers of $\mathcal{A}, \mathcal{M}$ at 0 . The space $W$ has a unique structure of a module over $V$, see Part $(a)$, such that the $\mathcal{A}_{V}$-module $\mathcal{M}_{W}$ is isomorphic to $\left.\mathcal{M}\right|_{\text {Spec } k[[t]]}$.

Thus, Proposition 6.1.2 gives the following.

(6.2.4) Theorem. The De Rham complex $\mathcal{C D R}\left(\omega_{X}\right)$ is a sheaf of vertex algebras on $X$. For any right $\mathcal{D}_{X}$-module $\mathcal{M}$ the De Rham complex $\mathcal{C D} \mathcal{R}(\mathcal{M})$ is a sheaf of $\operatorname{CD} \mathcal{R}\left(\omega_{X}\right)$-modules.

(6.3) The vacuum module and the chiral de Rham complex. Here we recall the original construction of the chiral de Rham complex of $X$ as given in [MSV]. One first considers the case $X=\mathbb{A}^{d}$. Similarly to Example 5.4.2, let $C D_{M}^{N}$ be the $\mathbb{Z} / 2 \mathbb{Z}$-graded $k$-algebra generated by even elements $a_{i n}, a_{i n}^{*}$ and odd elements $b_{i n}, b_{i n}^{*}$, with $i=1, \ldots, d$ and $-N \leq n \leq M$ for $a_{i n}, b_{i n}$ and $-M \leq n \leq N$ for $a_{i n}^{*}, b_{i n}^{*}$, modulo the relations

$$
\begin{array}{ll}
a_{i m}^{*} a_{j n}-a_{j n} a_{i m}^{*}=\delta_{i j} \delta_{m,-n}, & a_{i m} a_{j n}-a_{j n} a_{i m}=a_{i m}^{*} a_{j n}^{*}-a_{j n}^{*} a_{i m}^{*}=0, \\
b_{i m}^{*} b_{j n}+b_{j n} b_{i m}^{*}=\delta_{i j} \delta_{m,-n}, & b_{i m} b_{j n}+b_{j n} b_{i m}=b_{i m}^{*} b_{j n}^{*}+b_{j n}^{*} b_{i m}^{*}=0 .
\end{array}
$$

We further require that the letters $a$ and $b$ commute in all cases. Let $\tilde{C D}=$ $\lim _{M, N} C D_{M}^{N}$. Consider the super vector space $k^{d \mid d}$ with basis consisting of even vectors $v_{1}, \ldots v_{d}$ and odd vectors $v_{d+1}, \ldots v_{2 d}$. The space

$$
\tilde{\mathfrak{h}}=\left(k\left[t, t^{-1}\right] \oplus k\left[t, t^{-1}\right] d t\right) \otimes k^{d \mid d} \oplus k \gamma
$$


is then a Lie super algebra over $k$ with respect to the brackets given by

$$
\left[f \otimes v_{i}, \omega \otimes v_{j}\right]=\delta_{i j} \operatorname{Res}(f \cdot \omega) \gamma
$$

all other brackets being zero. It is clear that

$$
\tilde{C D}=U(\tilde{\mathfrak{h}}) /(\gamma-1)
$$

with $a_{i n} \mapsto v_{i} t^{n}, a_{i n}^{*} \mapsto v_{i} t^{n-1} d t, b_{i n} \mapsto v_{i+d} t^{n}, b_{i n}^{*} \mapsto v_{i+d} t^{n-1} d t$. Let also

$$
\mathfrak{h}=(k((t)) \oplus k((t)) d t) \otimes k^{d \mid d} \oplus k \gamma
$$

with the bracket defined in the same way, and

$$
C D=U(\mathfrak{h}) /(\gamma-1)
$$

Obviously $\tilde{C D} \subset C D$. Let

$$
\tilde{\mathfrak{h}}^{+}=(k[t] \oplus k[t] d t) \otimes k^{d \mid d}, \quad \mathfrak{h}^{+}=(k[[t]] \oplus k[[t]] d t) \otimes k^{d \mid d} .
$$

These are Abelian subalgebras in $\tilde{\mathfrak{h}}, \mathfrak{h}$. Set $\tilde{C D^{+}}=U\left(\tilde{\mathfrak{h}}^{+}\right) \tilde{C D}, C D^{+}=U\left(\mathfrak{h}^{+}\right) C D$. The vacuum module $\tilde{V a c}=\tilde{C D} / \tilde{C D}{ }^{+}, V a c=C D / C D^{+}$are identified as in (5.4.3)(a). We denote by $1 \in \operatorname{Vac}$ (the vacuum vector) the image of $1 \in C D$. As well-known, $V a c$ has a structure of a vertex algebra such that the generating series associated to the (-1)- and 0 -modes of $a_{i}, b_{i}$ are given by:

$$
\left(a_{i,-1} 1\right)(z)=\sum_{n \in \mathbb{Z}} a_{i n} z^{-n-1}, \quad\left(a_{i 0}^{*} 1\right)(z)=\sum_{n \in \mathbb{Z}} a_{i n}^{*} z^{-n}
$$

and similarly with $\left(b_{i,-1} 1\right)(z),\left(b_{i 0}^{*} 1\right)(z)$. The generating series associated to other modes are obtained by differentiation, using the action of $\partial$ given by

$$
\partial\left(a_{i n}\right)=n a_{i, n-1}, \quad \partial\left(a_{i n}^{*}\right)=(n-1) a_{i, n-1}^{*},
$$

and similarly for $b_{i n}^{*}, b_{i n}$. The map

$$
\delta=\sum_{i, n} a_{i n}^{*} b_{i,-n}: V a c \rightarrow V a c
$$

is a derivation of vertex algebras with zero square.

Setting $x_{i}=a_{i 0}$ makes $\operatorname{Vac}$ into a module over $k\left[x_{1}, \ldots, x_{n}\right]$, the coordinate ring of $\mathbb{A}^{d}$. We denote this ring shortly by $k[x]$. In [MSV] the authors consider the quasicoherent sheaf $\Omega_{\mathbb{A}^{d}}^{c h}:=V a c \otimes_{k[x]} \mathcal{O}_{\mathbb{A}^{d}}$ corresponding to $k[x]$-module $V a c$ and 
extend the vertex algebra structure to it. One also has a vertex algebra structure on

$$
V a c^{\wedge}:=\operatorname{Vac} \otimes_{k\left[x_{i}\right]} k\left[\left[x_{i}\right]\right] .
$$

Now let $X$ be a smooth algebraic variety, $U \subset X$ be an open subset and $\phi: U \rightarrow$ $\mathbb{A}^{d}$ be an étale map. Let $0 \in X$ be a point such that $\phi(0)=0$ and let $X^{\wedge}$ be the formal neighborhood of 0 in $X$. Then $x_{i}^{\prime}=\phi^{*} x_{i}$ are the coordinates on $X^{\wedge}$.

In [MSV] the authors construct a sheaf $\Omega_{X}^{c h}$ of differential vertex algebras on $X$ as the unique such sheaf satisfying the following condition. For any $\phi$ as above, there is an isomorphism of vertex algebras

$$
\phi_{c h}: \Omega_{X}^{c h} \otimes_{\mathcal{O}_{X}} \mathcal{O}_{X^{\wedge}} \rightarrow \phi^{*} \Omega_{\mathbb{A}^{d}}^{c h} \otimes_{\mathcal{O}_{\mathbb{A}^{d}}} \mathcal{O}_{\mathbb{A}^{d \wedge}}=V a c^{\wedge}
$$

which coincides, for $U \subset \mathbb{A}^{d}$, with the automorphism of $V a c^{\wedge}$ introduced in [MSV, Theorem 3.7]. Our aim in the rest of this paper is to prove the following fact.

(6.3.1) Theorem. There is an isomorphism of sheaves of differential vertex algebras $\Omega_{X}^{c h} \simeq \mathcal{C D} \mathcal{R}\left(\omega_{X}\right)$.

(6.4) The factorization algebra associated to the vacuum module. As the first step in proving Theorem 6.3.1, let us describe the factorization algebra corresponding to $\operatorname{Vac}=\Gamma\left(\mathbb{A}^{d}, \Omega_{\mathbb{A}^{d}}^{c h}\right)$. In this, we follow $[\mathrm{BD} 1],[\mathrm{G}]$ : the constructions below are a particular instance of the general concept of the chiral enveloping algebra of a Lie*-algebra. For the convenience of the reader we give a self-contained presentation.

Fix a smooth projective curve $C$. Recall that $\omega_{C}$ is the sheaf of 1-forms on $C$ (in the Zariski topology). Let $I$ be a finite set. Consider the product $C^{I} \times C$ and its projections $p, q$ to $C^{I}$ and $C$. Let us specialize the notation of (3.3) to the case when $S=C^{I}$ and $f_{I}=\operatorname{Id}: C^{I} \rightarrow C^{I}$. We denote the subvariety $\Gamma\left(f_{I}\right)$ simply by

$$
\Gamma_{I}=\left\{\left(\left(c_{i}\right), x\right) \in C^{I} \times C \mid x \in\left\{c_{i}\right\}\right\} .
$$

Similarly we write $\mathcal{O}_{I}^{\wedge}, \mathcal{K}_{I}^{\wedge}$ for $\mathcal{O}_{f_{I}}^{\wedge}, \mathcal{K}_{f_{I}}^{\wedge}$. Set

$$
\begin{gathered}
\mathcal{O}_{[[I]]}=p_{*} \mathcal{O}_{I}^{\wedge}, \quad \mathcal{O}_{((I))}=p_{*} \mathcal{K}_{I}^{\wedge}, \\
\omega_{[[I]]}=p_{*}\left(\mathcal{O}_{I}^{\wedge} \otimes q^{*} \omega_{C}\right), \quad \omega_{((I))}=p_{*}\left(\mathcal{K}_{I}^{\wedge} \otimes q^{*} \omega_{C}\right) .
\end{gathered}
$$

These are (non-quasicoherent) sheaves of $\mathcal{O}$-modules on $C^{I}$. Informally, the "fiber" of, say, $\omega_{((I))}$ at a point $\left(c_{i}\right) \in C^{I}$ is the space of sections of $\omega_{C}$ on the punctured formal neighborhood of the set $\left\{c_{i}\right\}$, and similarly in the other cases. Note that the sum of residues defines a morphism

$$
\operatorname{Res}_{\left(c_{i}\right)}: \omega_{((I))} \rightarrow \mathcal{O}_{C^{I}},
$$


trivial on $\omega_{[[I]]}$.

Consider the super-vector space $k^{d \mid d}$ as in (6.3). The sheaf

$$
\mathfrak{h}_{I}=\left(\mathcal{O}_{((I))} \oplus \omega_{((I))}\right) \otimes k^{d \mid d} \oplus \mathcal{O}_{C^{I}} \cdot \gamma
$$

is then a Lie superalgebra in the category of left $\mathcal{D}_{C^{I}}$-modules, with respect to the super-bracket

$$
\begin{aligned}
& {\left[\mathcal{O}_{C}^{d \mid d}, \mathcal{O}_{C}^{d \mid d}\right]=\left[\omega_{C}^{d \mid d}, \omega_{C}^{d \mid d}\right]=0,} \\
& {\left[v_{i} \otimes f, v_{j} \otimes \omega\right]=\delta_{i j} \operatorname{Res}_{\left(c_{i}\right)}(f \omega) \cdot \gamma, \quad \forall f \in \mathcal{O}_{C}, \forall \omega \in \omega_{C},}
\end{aligned}
$$

and with $\gamma$ being a central element. Similarly, let $\mathfrak{h}_{I}^{+} \subset \mathfrak{h}_{I}$ be the super-Lie subalgebra $\left(\mathcal{O}_{[[I]]} \oplus \omega_{[[I]]}\right) \otimes k^{d \mid d}$. For any surjective map $J \rightarrow I$ there are obvious isomorphisms

$$
\Delta^{(J / I) *} \mathfrak{h}_{J} \stackrel{\sim}{\rightarrow} \mathfrak{h}_{I}, \quad j^{(J / I) *}\left(\prod_{I} \mathfrak{h}_{J_{i}}\right) \stackrel{\sim}{\rightarrow} j^{(J / I) * \mathfrak{h}_{J}}
$$

Let $\mathcal{U}_{C^{I}}$ be the quotient of the associative enveloping algebra of $\mathfrak{h}_{I}$, in the category of left $\mathcal{D}_{C^{I}}$-modules, by the right ideal generated by $\gamma-1$. Consider the sheaf

$$
\mathcal{V} a c_{C^{I}}=\mathcal{U}_{C^{I}} / \mathcal{U}_{C^{I}}^{+}
$$

where $\mathcal{U}_{C^{I}}^{+} \subset \mathcal{U}_{C^{I}}$ is the right ideal generated by $\mathfrak{h}_{I}^{+}$. The collection $\left(\mathcal{V} a c_{C^{I}}\right)$ is clearly a factorization algebra. To simplify we may omit the subscript $C$, writing $\mathcal{V} a c$ instead of $\mathcal{V} a c_{C}$.

(6.4.1) Lemma. Vac is isomorphic, as a vertex algebra, to the fiber of the chiral algebra $\mathcal{V}$ ac at any point of $C$.

Proof: Fix a point $0 \in C$ and a formal coordinate $t$ at 0 . Let $D=\operatorname{Spec} k[[t]]$ be the formal neighborhood of 0 in $C$. We compute the chiral product, $\mu$, on the right $\mathcal{D}_{C}$-module $\mathcal{V} a c^{r}:=\mathcal{V} a c \otimes_{\mathcal{O}_{C}} \omega_{C}$. The scheme $C \times C$ is equipped with the coordinates $t:=t \otimes 1, z:=1 \otimes t$. The $\mathcal{O}_{C}$-module $\mathfrak{h}_{\{1\}}$ is locally free, such that

$$
\Gamma\left(D, \mathfrak{h}_{\{1\}}\right)=\left(k^{d \mid d} \oplus k^{d \mid d} d z\right)[[z-t, t]]\left[(z-t)^{-1}\right] \oplus k[[t]] \cdot \gamma
$$

Thus the map

$$
\begin{aligned}
(z-t)^{m} v_{i} & \mapsto a_{i m}, \quad(z-t)^{m} v_{i+d} \mapsto b_{i m}, \\
(z-t)^{m-1} v_{i} d z & \mapsto a_{i m}^{*}, \quad(z-t)^{m-1} v_{i+d} d z \mapsto b_{i m}^{*}
\end{aligned}
$$

extends uniquely to an isomorphism of $k[[t]]$-vector spaces $\Gamma\left(D, \mathcal{U}_{C}\right) \stackrel{\sim}{\rightarrow} C D[[t]]$, where $C D$ was defined in (6.3). Let $1,1_{C}, 1_{C^{2}}$ be the vacuum elements of $V a c, \mathcal{V} a c$, 
$\mathcal{V} a c_{C^{2}}$. We consider the unique isomorphism of $C D[[t]]$-modules $\Gamma(D, \mathcal{V} a c) \stackrel{\sim}{\rightarrow} \operatorname{Vac}[[t]]$ such that $1_{C} \mapsto 1$.

The scheme $C^{2} \times C$ is equipped with the local coordinates $t_{i}:=t_{i} \otimes 1, z:=1 \otimes t$, $i=1,2$. Put $R=k\left[\left[t_{1}, t_{2}\right]\right]$. The $\mathcal{O}_{C^{2}}$-module $\mathfrak{h}_{\{1,2\}}$ is locally free, such that

$$
\Gamma\left(D^{2}, \mathfrak{h}_{\{1,2\}}\right)=\left(R^{d \mid d} \oplus R^{d \mid d} d z\right)\left[\left[z-t_{1}, z-t_{2}\right]\right]\left[\left(z-t_{1}\right)^{-1},\left(z-t_{2}\right)^{-1}\right] \oplus R \cdot \gamma
$$

Let $T_{1}$ (resp. $T_{2}$ ) be the Taylor expansion

$$
R\left[\left[z-t_{1}, z-t_{2}\right]\right]\left[\left(z-t_{1}\right)^{-1},\left(z-t_{2}\right)^{-1},\left(t_{1}-t_{2}\right)^{-1}\right] \rightarrow R\left(\left(z-t_{2}\right)\right)\left(\left(z-t_{1}\right)\right)
$$

(resp. $\left.R\left(\left(z-t_{1}\right)\right)\left(\left(z-t_{2}\right)\right)\right)$. The factorization map

$$
j \bullet j \bullet\left(\mathfrak{h}_{\{1,2\}}\right) \stackrel{\sim}{\rightarrow} j \bullet j^{\bullet}\left(\mathfrak{h}_{\{1\}} \times \mathfrak{h}_{\{1\}}\right)
$$

takes an element $a \in \Gamma\left(D^{2}, j_{\bullet} j^{\bullet}\left(\mathfrak{h}_{\{1,2\}}\right)\right)$ to $\left(T_{1}(a), T_{2}(a)\right)$. It induces an action of the sheaf of Lie algebras $j_{\bullet} j^{\bullet}\left(\mathfrak{h}_{\{1,2\}}\right)$ on $j_{\bullet} j^{\bullet}\left(\mathcal{V} a c^{r} \otimes \mathcal{V} a c^{r}\right)$. The factorization map

$$
j \cdot j^{\bullet}\left(\mathcal{V} a c^{r} \otimes \mathcal{V} a c^{r}\right) \stackrel{\sim}{\rightarrow} j \bullet j^{\bullet}\left(\mathcal{V} a c_{C^{2}}^{r}\right)
$$

is the unique morphism of sheaves of $j_{\bullet} j^{\bullet}\left(\mathfrak{h}_{\{1,2\}}\right)$-modules taking $1_{C} \otimes 1_{C}$ to $1_{C^{2}}$. The chiral product $\mu$ is the composition of the chain of maps

$j_{\bullet} j^{\bullet}\left(\mathcal{V} a c^{r} \otimes \mathcal{V} a c^{r}\right) \stackrel{\sim}{\rightarrow} j_{\bullet} j^{\bullet}\left(\mathcal{V} a c_{C^{2}}^{r}\right) \rightarrow j \bullet j^{\bullet}\left(\mathcal{V} a c_{C^{2}}^{r}\right) / \mathcal{V} a c_{C^{2}}^{r}=\Delta_{\bullet} \Delta^{\bullet}\left(\mathcal{V} a c_{C^{2}}^{r}\right)=\Delta_{\bullet}\left(\mathcal{V} a c^{r}\right)$.

The right $\Gamma\left(D^{2}, \mathcal{D}_{C^{2}}\right)$-module $\Gamma\left(D^{2}, \Delta_{\bullet}\left(\mathcal{V} a c^{r}\right)\right)$ is spanned by the symbols $a(t) \delta\left(t_{1}-\right.$ $\left.t_{2}\right)$, for any $a(t) \in \Gamma\left(D, \mathcal{V} a c^{r}\right)$, modulo the relations

$$
\begin{gathered}
\left(a(t) \delta\left(t_{1}-t_{2}\right)\right)\left(\partial_{t_{1}}+\partial_{t_{2}}\right)=\left(a(t) \partial_{t}\right) \delta\left(t_{1}-t_{2}\right), \\
\left(a(t) \delta\left(t_{1}-t_{2}\right)\right) f\left(t_{1}, t_{2}\right)=(a(t) f(t, t)) \delta\left(t_{1}-t_{2}\right),
\end{gathered}
$$

for any $f\left(t_{1}, t_{2}\right) \in k\left[\left[t_{1}, t_{2}\right]\right]$. Fix $b \in \Gamma\left(D, \mathcal{V} a c^{r}\right)$. Note that

$$
T_{2}\left(\left(z-t_{1}\right)^{-1}\right)=-\sum_{m \geq 0}\left(t_{1}-t_{2}\right)^{-m-1}\left(z-t_{2}\right)^{m} .
$$

Hence,

$$
\begin{aligned}
\mu\left(\left(t_{1}-t_{2}\right)^{n}\left(a_{i,-1} 1_{C}\right) \otimes b\right) & =\mu\left(\left(a_{i,-1} 1_{C}\right) \otimes b\right)\left(t_{1}-t_{2}\right)^{n} \\
& =\sum_{m \in \mathbb{Z}} a_{i m} b \delta\left(t_{1}-t_{2}\right) \partial_{t_{2}}^{(m-n)} .
\end{aligned}
$$


Similarly, we get

$$
\begin{gathered}
\mu\left(\left(t_{1}-t_{2}\right)^{n}\left(b_{i,-1} 1_{C}\right) \otimes b\right)=\sum_{m \in \mathbb{Z}} b_{i m} b \delta\left(t_{1}-t_{2}\right) \partial_{t_{2}}^{(m-n)}, \\
\mu\left(\left(t_{1}-t_{2}\right)^{n}\left(a_{i 0}^{*} 1_{C}\right) \otimes b\right)=\sum_{m \in \mathbb{Z}} a_{i m}^{*} b \delta\left(t_{1}-t_{2}\right) \partial_{t_{2}}^{(m-n)}, \\
\mu\left(\left(t_{1}-t_{2}\right)^{n}\left(b_{i 0}^{*} 1_{C}\right) \otimes b\right)=\sum_{m \in \mathbb{Z}} b_{i m}^{*} b \delta\left(t_{1}-t_{2}\right) \partial_{t_{2}}^{(m-n)}
\end{gathered}
$$

On the other hand, the chiral product associated to the vertex algebra $V[[t]]$ is the map

$$
V a c \otimes V a c\left[\left[t_{1}, t_{2}\right]\right]\left[\left(t_{1}-t_{2}\right)^{-1}\right] \rightarrow \operatorname{Vac}\left[\left[t_{1}, t_{2}\right]\right]\left[\left(t_{1}-t_{2}\right)^{-1}\right] / V a c\left[\left[t_{1}, t_{2}\right]\right]
$$

taking $\left(t_{1}-t_{2}\right)^{n}\left(a_{i,-1} 1\right) \otimes b$ to

$$
\sum_{m \in \mathbb{Z}} a_{i m} b \partial_{t_{2}}^{(m-n)} \delta\left(t_{1}-t_{2}\right)
$$

where $\partial_{t_{2}}^{(m)} \delta\left(t_{1}-t_{2}\right)$ stands for the element $\left(t_{1}-t_{2}\right)^{-m-1}+k\left[\left[t_{1}, t_{2}\right]\right]$, and similarly for $b_{i,-1}, a_{i 0}^{*}, b_{i 0}^{*}$. Thus, to prove that the chiral algebra $\mathcal{V} a c^{r}$ is isomorphic to the chiral algebra on $D$ built from $V a c$ as in (6.2) it is sufficient to check that the corresponding right $\mathcal{D}_{C}$-modules coincide. See [BD1, Remark 3.4.8.(i)] for an elementary definition of the the canonical left $\mathcal{D}_{C}$-module structure on $\mathcal{V} a c$. By construction we have $\partial_{t}\left(1_{C}\right)=0$. It is easy to see that $\partial_{t}\left(a_{i m} 1_{C}\right)=m a_{i, m-1} 1_{C}$ for all $m<0$. Hence, the operators $\partial$ on $\Gamma\left(D, \mathcal{V} a c^{r}\right)$ and $V a c[[t]]$ coincide on $a_{i m}$. The case of $a_{i m}^{*}, b_{i m}^{*}, b_{i m}$ is similar.

(6.5) The action of étale morphisms I. To prove Theorem 6.3.1 in full generality, it suffices to establish the following lemma. Let $U \subset X$ be any affine open set and $\phi: U \rightarrow \mathbb{A}^{d}$ be any étale map. Fix a point $0 \in U$ such that $\phi(0)=0$. Let $X^{\wedge}$ be the formal neighborhood of 0 . In particular we write $\mathbb{A}^{d, \wedge}$ for $\left(\mathbb{A}^{d}\right)^{\wedge}$.

(6.5.1) Lemma. (a) There is an isomorphism of differential vertex algebras $F_{\phi}$ : $\phi^{*} \Omega_{\mathbb{A}^{d, \wedge}}^{c h} \stackrel{\sim}{\rightarrow} k\left[X^{\wedge}\right] \otimes_{\mathcal{O}_{X}} \mathcal{C D R}\left(\omega_{X}\right)$.

(b) If $X=\mathbb{A}^{d}$ then $F_{\phi}^{-1} \circ F_{\mathrm{Id}}=\phi_{c h}$ is the isomorphism constructed in [MSV].

The plan of the proof is as follows. We will construct an isomorpism $\left(F_{I}\right)$ of factorization algebras and obtain $F_{\phi}$ as the fiber of $F_{\{1\}}$ at a point of $C$. It is enough to assume that $C=\mathbb{A}^{1}$. Set

$$
C D_{I}=\Gamma\left(\mathbb{A}^{I}, \mathcal{U}_{C^{I}}\right), \quad C D_{I}^{+}=\Gamma\left(\mathbb{A}^{I}, \mathcal{U}_{C^{I}}^{+}\right)
$$




$$
\operatorname{DR}\left(\omega_{U}\right)_{I}=\Gamma\left(\mathcal{L}(U)_{\mathbb{A}^{I}}, \mathcal{C D} \mathcal{R}\left(\omega_{U}\right)_{\mathbb{A}^{I}}\right), \quad \operatorname{Vac}_{I}=\Gamma\left(\mathbb{A}^{I}, \mathcal{V} a c_{C^{I}}\right)
$$

Thus, $V a c_{I}=C D_{I} / C D_{I}^{+}$. If $X=\mathbb{A}^{d}$ we introduce the algebra

$$
\tilde{C D_{I}}=\lim _{n, N} C D_{X_{n}^{N}}
$$

where

$$
A=k\left[\mathbb{A}^{I}\right], \quad A_{n}^{N}=A\left[a_{l \nu}^{(j)} ;-N \leq l \leq n\right], \quad X_{n}^{N}=\operatorname{Spec} A_{n}^{N},
$$

see Example 5.4.2. Note that $C \tilde{D}_{I}$ is a subalgebra of $C D_{I}$. Let

$$
\tilde{C D_{I}^{+}}=\lim _{n, N} C D_{X_{n}^{N}}^{+}
$$

where $C D_{X_{n}^{N}}^{+}$is the right ideal generated by $a_{l \nu}^{(j)}, a_{l+1, \nu}^{(j) *}, b_{l \nu}^{(j)}, b_{l+1, \nu}^{(j) *}$ with $l \geq 0$ and $\tilde{V a c_{I}}=\tilde{C D_{I}} / \tilde{C D_{I}^{+}}$.

(6.5.3) Lemma. $\tilde{V a c_{I}}$ is an irreducible $\tilde{C D_{I}}$-module and the natural map of vector spaces $\tilde{V_{a c}} \rightarrow V_{I} c_{I}$ is an isomorphism.

Proof: Irreducibility follows from the fact that $C D_{X_{n}^{N}} / C D_{X_{n}^{N}}^{+}$is irreducible over $C D_{X_{n}^{N}}$. The isomorphism follows from the normal form of elements of $V a c_{I}$ and $\tilde{V a c}{ }_{I}$.

Now, to prove Lemma (6.5.1) we will construct a right action

$$
D R\left(\omega_{U}\right)_{I} \otimes \tilde{C D} D_{I} \rightarrow D R\left(\omega_{U}\right)_{I}
$$

commuting with the factorization maps. To prove that the factorization algebras $\left(\mathcal{V} a c_{\mathbb{A}^{I}}\right),\left(\mathcal{C D} \mathcal{R}\left(\omega_{U}\right)_{\mathbb{A}^{I}}\right)$ are isomorphic, it is then sufficient to check that the right $\tilde{C D_{I}}$-module $D R\left(\omega_{U}\right)_{I}$ has a cyclic vector whose annihilator is $\tilde{C D}+$. Observe that, since the map (6.5.3) depends on $\phi$, the resulting isomorphism of sheaves of vertex algebras $\phi^{*} \Omega_{\mathbb{A}^{d, \wedge}}^{c h} \stackrel{\sim}{\rightarrow} k\left[X^{\wedge}\right] \otimes_{\mathcal{O}_{X}} \mathcal{C D} \mathcal{R}\left(\omega_{X}\right)$ will also depend on $\phi$.

(6.5.5) The case $U=\mathbb{A}^{d}$. First we consider the particular case where $U=\mathbb{A}^{d}$ and $\phi=\mathrm{Id}$. What we do in this case is to provide an explicit identification of the

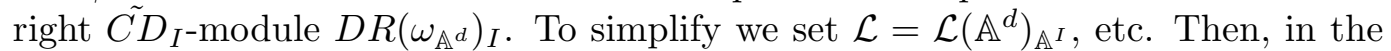
notations of (6.5.2),

$\mathcal{L}_{n}=\lim _{N} \operatorname{Spf}\left(A_{n}^{0}\left[\left[a_{l \nu}^{(j)} ;-N \leq l<0\right]\right]\right), \quad \mathcal{L}_{n}^{\varepsilon}=\operatorname{Spec}\left(A_{n}^{N_{\varepsilon}} /\left(a_{l \nu_{1}}^{(j)} \cdots a_{l \nu_{1+\varepsilon_{l}}}^{(j)} ; l<0\right)\right)$, 
see (3.9.1), where we set $N_{\varepsilon}=\max \left\{l ; \varepsilon_{-l} \neq 0\right\}$ for each $\varepsilon \in \mathbb{E}$. To simplify again we set $X_{n}^{\varepsilon}=X_{n}^{N_{\varepsilon}}$. Thus $X_{N}^{\varepsilon}$ is an affine space of finite dimension. There are closed embeddings $\mathcal{L}_{n}^{0} \subset \mathcal{L}_{n}^{\varepsilon} \subset X_{n}^{\varepsilon}$. Let $i_{n \varepsilon}: \mathcal{L}_{n}^{0} \hookrightarrow X_{n}^{\varepsilon}$ be the composite embedding. We write $\omega_{\mathcal{L}_{n}^{0}, X_{n}^{\varepsilon}}$ for the right $\mathcal{D}$-module $i_{n \varepsilon} \bullet \omega_{\mathcal{L}_{n}^{0}}$ on $X_{n}^{\varepsilon}$. Let $\mathcal{D} \mathcal{R}\left(\omega_{n}^{\varepsilon}\right) \in \mathbf{O}_{\mathcal{L}_{n}^{\varepsilon}}$ be the subsheaf of $\mathcal{D} \mathcal{R}\left(\omega_{\mathcal{L}_{n}^{0}, X_{n}^{\varepsilon}}\right)$ consisting of the sections supported (scheme-theoretically) on $\mathcal{L}_{n}^{\varepsilon}$. By definition,

$$
\lim _{\varepsilon} \Gamma\left(\mathcal{L}_{n}^{\varepsilon}, \mathcal{D} \mathcal{R}\left(\omega_{n}^{\varepsilon}\right)\right)={\underset{\lim }{\longrightarrow}}_{N} \Gamma\left(\mathcal{L}_{n}^{0}, \mathcal{D} \mathcal{R}\left(\omega_{\mathcal{L}_{n}^{0}, X_{n}^{N}}\right)\right)=D R_{\mathcal{L}_{n}^{0}, X_{n}^{\infty}}
$$

Let us denote this space by $D R\left(\omega_{n}\right)_{I}$. Hence,

$$
D R\left(\omega_{\mathbb{A}^{d}}\right)_{I}=\lim _{n} D R\left(\omega_{n}\right)_{I}[n d],
$$

and there is a right $\lim _{N} C D_{X_{n}^{N}}$-action on $D R\left(\omega_{n}\right)_{I}$, such that $D R\left(\omega_{n}\right)_{I}$ is the quotient of $\lim _{N} C D_{X_{n}^{N}}$ by $\lim _{N} C D_{X_{n}^{N}}^{+}$. Using (6.5.4) we get a right action of $\tilde{C D_{I}}$ on $D R\left(\omega_{\mathbb{A}^{d}}\right)_{I}$ such that

$$
\operatorname{DR}\left(\omega_{\mathbb{A}^{d}}\right)_{I} \simeq \tilde{C D_{I}} / \tilde{C D_{I}^{+}} \simeq \tilde{\operatorname{Vac}}{ }_{I}=V a c_{I},
$$

thus achieving our goal in the case $X=\mathbb{A}^{d}$.

(6.5.7) Corollary. Theorem 6.3.1 is true for $X=\mathbb{A}^{d}$.

(6.6) Etale change of coordinates in Clifford algebras. In order to prove Lemma 6.5.1 for general $\phi: U \rightarrow \mathbb{A}^{d}$ we need some elementary observations about Clifford algebras.

If $X$ is a smooth algebraic variety, we denote by $\mathcal{C} \mathcal{D}_{X}$ the sheaf of differential operators in $\Omega_{X}^{\bullet}$, the commutative superalgebra of differential forms.

In particular, if $X=\mathbb{A}^{d}$ with coordinates $x_{1}, \ldots, x_{d}$, then $x_{i}, d x_{i}$ are free generators of (the algebra of global sections of) $\Omega_{X}^{\bullet}$ and we denote $\partial_{i}=\partial / \partial x_{i}$ and $\xi_{i}=" \partial / \partial d x_{i}$ " the corresponding derivations, which are thus global sections of $\mathcal{C D}_{\mathbb{A}^{d}}$.

Let now $U$ be an affine open subset of a smooth variety $X$, and let $\phi: U \rightarrow \mathbb{A}^{d}$ be an étale map. Let $x_{i}^{\prime}=\phi^{*} x_{i}$ be the coordinate on $X^{\wedge}$. There are then uniquely determined derivations $\partial_{i}^{\prime}, \xi_{i}^{\prime}$ of $\Omega_{X^{\wedge}}$ such that

$$
\left[\partial_{i}^{\prime}, x_{j}^{\prime}\right]=\left[\xi_{i}^{\prime}, d x_{j}^{\prime}\right]=\delta_{i j}, \quad\left[\partial_{i}^{\prime}, d x_{j}^{\prime}\right]=\left[\xi_{i}^{\prime}, x_{j}^{\prime}\right]=0
$$

Set $C D_{X^{\wedge}}=k\left[X^{\wedge}\right] \hat{\otimes}_{\mathcal{O}_{X}} \mathcal{C} \mathcal{D}_{X}$. The étale map $\phi$ gives an isomorphism of formal schemes $X^{\wedge} \stackrel{\sim}{\rightarrow} \mathbb{A}^{d, \wedge}$. Let $\phi^{\prime}$ be the inverse isomorphism. 
(6.6.1) Lemma. For any $\phi$ as above, there is a unique $k\left[X^{\wedge}\right]$-algebra isomorphism

$$
\phi_{\sharp}: C D_{X^{\wedge}} \stackrel{\sim}{\rightarrow} \phi^{*} C D_{\mathbb{A}^{d, \wedge}}
$$

such that

$$
\begin{gathered}
\phi_{\sharp}\left(d x_{i}^{\prime}\right)=\sum_{j} \partial_{j} \phi_{i}(x) d x_{j}, \quad \phi_{\sharp}\left(\xi_{i}^{\prime}\right)=\sum_{j} \partial_{i}^{\prime} \phi_{j}^{\prime}(\phi(x)) \xi_{j}, \\
\phi_{\sharp}\left(\partial_{i}^{\prime}\right)=\sum_{j} \partial_{i}^{\prime} \phi_{j}^{\prime}(\phi(x)) \partial_{j}+\sum_{j, k, l} \partial_{i}^{\prime} \partial_{k}^{\prime} \phi_{j}^{\prime}(\phi(x)) \partial_{l} \phi_{k}(x) d x_{k} \xi_{j},
\end{gathered}
$$

where $\phi=\left(\phi_{1}, \ldots, \phi_{d}\right)$.

Proof: It suffices to observe that, since the map $\phi$ is étale, $C D_{X^{\wedge}}$ is a free $k\left[X^{\wedge}\right]$ module with basis

$$
\left(\partial_{1}^{\prime}\right)^{r_{1}}\left(\partial_{2}^{\prime}\right)^{r_{2}} \cdots\left(\partial_{d}^{\prime}\right)^{r_{d}} \otimes\left(\xi_{1}^{\prime}\right)^{m_{1}} \cdots\left(\xi_{d}^{\prime}\right)^{m_{d}}\left(d x_{1}^{\prime}\right)^{n_{1}} \cdots\left(d x_{d}^{\prime}\right)^{n_{d}},
$$

where $r_{i}, m_{i}, n_{i} \in \mathbb{N}$. Then use the coordinates change formulas, see [L, chap. II] for instance.

(6.7) The action of étale morphisms II. Let now $\phi: U \rightarrow \mathbb{A}^{d}$ be a general étale morphism, with $U$ affine. By (3.9.2) we have an isomorphism of schemes

$$
\mathcal{L}_{n}^{\varepsilon}(\phi)_{\mathbb{A}^{I}} \simeq \mathcal{L}_{n}^{\varepsilon} \times \mathcal{L}_{0}^{0} \mathcal{L}_{0}^{0}(U)_{\mathbb{A}^{I}}, \quad \text { where } \mathcal{L}_{n}^{\varepsilon}=\mathcal{L}_{n}^{\varepsilon}\left(\mathbb{A}^{d}\right)_{\mathbb{A}^{I}}
$$

We will use freely the notations in (6.5), (6.6). There is an obvious map $X_{n}^{N} \rightarrow \mathcal{L}_{0}^{0}$, since $\mathcal{L}_{0}^{0}=X_{0}^{0}=\operatorname{Spec} A_{0}^{0}$, which restricts to the map $\mathcal{L}_{n}^{\varepsilon} \rightarrow \mathcal{L}_{0}^{0}$ when $N=N_{\varepsilon}$. For any $N$, consider the fiber product

$$
X_{n \phi}^{N}=X_{n}^{N} \times \mathcal{L}_{0}^{0} \mathcal{L}_{0}^{0}(U)_{\mathbb{A}^{I}}
$$

Denote by $\phi_{n}^{N}: X_{n \phi}^{N} \rightarrow X_{n}^{N}$ the projection to the first factor. Being a base change of an étale morphism $\mathcal{L}_{0}^{0}(\phi)_{\mathrm{Id}}: \mathcal{L}_{0}^{0}(U)_{\mathbb{A}^{I}} \rightarrow \mathcal{L}_{0}^{0}$, see Proposition 3.9.3, the map $\phi_{n}^{N}$ is étale. Set $0=\left\{a_{0 \nu}^{(j)}=0\right\} \in \operatorname{Hom}_{\mathbf{S c h}}\left(\mathbb{A}^{I}, X_{0}^{0}\right)$. Fix $0 \in \operatorname{Hom}_{\mathbf{S c h}}\left(\mathbb{A}^{I}, X_{0 \phi}^{0}\right)$ mapping to 0 by $\phi_{n}^{N}$. Let $X_{0}^{0, \wedge}, X_{0 \phi}^{0, \wedge}$ be the formal neighborhoods of 0 , and set

$$
X_{n}^{N, \wedge}=X_{0}^{0, \wedge} \times_{X_{0}^{0}} X_{n}^{N}, \quad X_{\phi}^{N, \wedge}=X_{0 \phi}^{0, \wedge} \times_{X_{0}^{0}} X_{n}^{N}
$$

By Lemma 6.6.1 applied to $\phi_{n}^{N}$, we have a ring isomorphism

$$
\phi_{n \sharp}^{N}: C D_{X_{n \phi}^{N, \wedge}} \stackrel{\sim}{\rightarrow} C D_{X_{n}^{N}, \wedge} .
$$


Let

$$
\tilde{C D_{I}^{\wedge}}=\underline{\lim }_{n, N} C D_{X_{n}^{N}, \wedge}, \tilde{C D_{I, \phi}^{\wedge}}=\lim _{n, N} C D_{X_{n \phi}^{N, \wedge}}
$$

so that the $\phi_{n \sharp}^{N}$ give a ring isomorphism

$$
\phi_{\infty \sharp}^{\infty}: \tilde{C D_{I, \phi}^{\wedge}} \rightarrow \tilde{C D_{I}^{\wedge}}
$$

Let also

$$
\tilde{C D_{I}^{\wedge,+}}=\lim _{n, N} C D_{X_{n}^{N, \wedge}}^{+},
$$

where

$$
C D_{X_{n}^{N, \wedge}}^{+}=k\left[X_{n}^{N, \wedge}\right] \otimes_{k\left[X_{n}^{N}\right]} C D_{X_{n}^{N}}^{+}
$$

and $C D_{X_{n}^{N}}^{+}$is introduced after (6.5.2). We have then the vacuum modules

$$
V a c_{I}^{\wedge}=\tilde{C D_{I}^{\wedge}} / \tilde{C D_{I}^{\wedge,+}}
$$

which form the factorization algebra corresponding to the vertex algebra $V a c^{\wedge}$ defined in (6.3). Note that

$$
D R\left(\omega_{U}\right)_{I}=\lim _{n, N} D R\left(\omega_{\mathcal{L}_{n}^{0}(U)_{\mathbb{A}}, X_{n \phi}^{N}}^{N}\right)[n d],
$$

and after tensoring with $k\left[U^{\wedge}\right]$ we get a module $D R\left(\omega_{U^{\wedge}}\right)_{I}$ over $\tilde{C D_{I, \phi}^{\wedge}}$.

(6.7.2) Lemma. With respect to the above structure of a $\tilde{C D_{I}^{\wedge}}$-module, $D R\left(\omega_{U^{\wedge}}\right)_{I}$ is isomorphic to $\operatorname{Vac}_{I}^{\wedge}$.

Proof: Follows from the fact that each $D R\left(\omega_{\mathcal{L}_{n}^{0}(U), X_{n \phi}^{N}}\right)$ is isomorphic to the vacuum module over $C D_{X_{n \phi}^{N}}$.

Note that both $D R\left(\omega_{U^{\wedge}}\right)_{I}$ and $V a c_{I}^{\wedge}$ have distinguished generators. Namely $D R\left(\omega_{U^{\wedge}}\right)_{I}$ is the limit of an inductive system with the first term $D R\left(\omega_{\mathcal{L}_{0}^{0}(U)_{\mathbb{A}^{I}} / \mathbb{A}^{I}}\right)$. But for any smooth morphism $Z \rightarrow S$ there is a canonical element $1_{Z / S}$ in $D R\left(\omega_{Z / S}\right)$ and we take $1_{I}^{\prime} \in D R\left(\omega_{U^{\wedge}}\right)_{I}$ to be the image of $1_{\mathcal{L}_{0}^{0}(U)_{\mathbb{A}^{I}} / \mathbb{A}^{I}}$ in the limit. The generator $1_{I}^{\prime \prime} \in V a c_{I}^{\wedge}$ is the image of $1 \in \tilde{C D_{I}^{\wedge}}$. We denote $F_{I}: V a c_{I}^{\wedge} \rightarrow D R\left(\omega_{U^{\wedge}}\right)_{I}$ the unique module isomorphism taking $1_{I}^{\prime \prime}$ to $1_{I}^{\prime}$. Let $\mathcal{F}_{I}: \mathcal{V} a c_{I}^{\wedge} \rightarrow \mathcal{D} \mathcal{R}\left(\omega_{U^{\wedge}}\right)_{I}$ be the corresponding morphism of quasicoherent sheaves on $\mathcal{L}_{0}^{0}(U)_{\mathbb{A}^{I}}$.

(6.7.3) Lemma. The $\left(\mathcal{F}_{I}\right)$ commute with factorization maps and thus form an isomorphism of factorization algebras. 
Proof: Follows from the fact that $\left(1_{I}^{\prime}\right)$ and $\left(1_{I}^{\prime \prime}\right)$ are compatible with factorization structures: in the notation of (6.1.1) we have $\varkappa^{J / I}\left(\nabla 1_{J_{i}}^{\prime}\right)=1_{J}^{\prime}$ and similarly for $1_{I}^{\prime \prime}$.

Set $I=\{1\}$. Let $F_{\phi}$ be the fiber of $F_{\{1\}}$ at the point $0 \in \mathbb{A}^{1}$. Lemma 6.7.3 implies that $F_{\phi}$ is a morphism of vertex algebras. This establishes part (a) of Lemma 6.5.1

We now prove Lemma 6.5.1(b). So we assume $U \subset \mathbb{A}^{d}$ and need to compare two automorphisms of the vertex algebra $\Gamma(U, \mathcal{V} a c)$, namely $F_{\phi}^{-1} \circ F_{\mathrm{Id}}$ and $\phi_{c h}$. Notice that the vertex algebra $V a c$ is strongly generated by the fields $\left(a_{i,-1} 1\right)(z),\left(a_{i 0}^{*} 1\right)(z)$, $\left(b_{i,-1} 1\right)(z),\left(b_{i 0}^{*} 1\right)(z)$, see $[\mathrm{K}]$, and that $\Gamma(U, \mathcal{V} a c)$ is obtained by localization. Thus it is enough to compare the two automorpisms on the elements

$$
\left(a_{i,-1} 1\right),\left(a_{i 0}^{*} 1\right),\left(b_{i,-1} 1\right),\left(b_{i 0}^{*} 1\right) \in \text { Vac. }
$$

Since $U \subset \mathbb{A}^{d}$, the sheaf of algebras $\tilde{C D_{I, \phi}^{\wedge}}$ is identified with $\tilde{C D_{I}^{\wedge}}$, so $\phi_{\infty, \sharp}^{\infty}$ is an automorphism of the latter.

Lemma. (a) The morphism $\phi_{\infty \sharp}^{\infty}$ preserves $\tilde{C D}_{I}^{\wedge,+}$ and thus induces an automorphism $\phi_{\sharp, I}: V a c_{I}^{\wedge} \rightarrow V a c_{I}^{\wedge}$.

(b) For $I=\{\bullet\}$, the morphism of vertex algebras $F_{\phi}^{-1} \circ F_{\text {Id }}: V a c^{\wedge} \rightarrow V a c^{\wedge}$ is equal to $\phi_{\sharp, 0}$, which is the fiber over $0 \in \mathbb{A}^{1}$ of the morphism $\phi_{\sharp, I}$.

Proof: (a) is enough to verify for each $\phi_{n \sharp}^{N}: C D_{X_{n \phi}^{N, \wedge}} \rightarrow C D_{X_{n \phi}^{N, \wedge}}$, in which case it follows from Lemma 6.6.1. Claim (b) follows from construction of $F_{\phi}$.

To prove $(6.5 .1)(b)$ it suffices therefore to check that $\phi_{\sharp 0}=\phi_{c h}$ on elements (6.7.4). Recall that $\mathcal{L}_{0}^{0}(X)_{\mathbb{A}^{1}}=X \times \mathbb{A}^{1}$ for any $X$. Hence, there is a commutative diagram

$$
\begin{array}{ccccc}
X_{n \phi}^{N} \rightarrow & X_{0 \phi}^{0} & = & U \times \mathbb{A}^{1} \\
\phi_{n}^{N} \downarrow & & \downarrow & & \downarrow \phi \\
X_{n}^{N} \rightarrow & \rightarrow & X_{0}^{0} & = & \mathbb{A}^{d} \times \mathbb{A}^{1} .
\end{array}
$$

This diagram induces a diagram of $A$-algebra homomorphisms

$$
\begin{array}{ccc}
C D_{Y_{\phi}^{\wedge}} & \hookleftarrow & C D_{X_{0 \phi}^{0 \wedge}} \\
\phi_{\sharp} \downarrow & & \downarrow \phi_{0 \sharp}^{0} \\
C D_{Y^{\wedge}} & \hookleftarrow & C D_{X_{0}^{0 \wedge}}^{0 \wedge} .
\end{array}
$$

Note that the images of the elements (6.7.4) by $\phi_{\sharp}$ and $\phi_{0 \sharp}^{0}$ coincide, modulo the identification

$$
a_{i 0} \mapsto x_{i}, \quad b_{i 0} \mapsto d x_{i}, \quad b_{i,-1}^{*} \mapsto \xi_{i}, \quad a_{i,-1}^{*} \mapsto \partial_{i} .
$$

On the other hand the images of the elements (6.7.4) by $\phi_{c h}$ and $\phi_{0 \sharp}^{0}$ coincide, see the formulas [MSV, (3.17)] for $\phi_{c h}$, and Lemma 6.6.1 for $\phi_{0 \sharp}^{0}$. We are done. 


\section{REFERENCES}

[AM] Artin, M., Mazur, B., Etale Homotopy, Lecture Notes in Math, vol. 100, Springer, 1970.

[B] Bakalov, B., Beilinson-Drinfeld's definition of a chiral algebra, available from http://www. math.berkeley.edu/ bakalov/.

[BB] Beilinson, A., Bernstein, I., A proof of the Jantzen conjectures, I.M. Gelfand Seminar (S. Gelfand, S. Gindikin, ed.), vol. 1, pp. 1-50.

[BD1] Beilinson, A., Drinfeld, V., Chiral algebras, available from http://zaphod.uchicago.edu/ benzvi/.

[BD2] Beilinson, A., Drinfeld, V., Quantization of Hitchin's integrable system and Hecke eigensheaves, available from http://zaphod.uchicago.edu/ benzvi/

[BLR] Bosch, S., Lütkebohmert, W., Raynaud, M., Néron models, Springer-Verlag, 1990.

[CC] Contou-Carrère, C., Jacobienne locale, groupe de bivecteurs de Witt universel et symbole modéré, C.R. Acad. Sci. Paris Sér. I Math. 318 (1994), 743-746.

[DL] Denef, J., Loeser, F., Germs of arcs on singular algebraic varieties and motivic integration, Invent. Math. 135 (1999), 201-232.

[EGA0] Grothendieck, A., Dieudonné, J., Eléments de géométrie algébrique, Grund. Math. Wiss., vol. 166, Springer, Boston-Basel-Berlin, 1971.

[EGAIV] Grothendieck, A., Dieudonné, J., Eléments de géométrie algébrique IV, Publ. Math. IHES, 1967.

[F] Frenkel, E., Vertex algebras and algebraic curves, Séminaire Bourbaki, Éxp. 875, Juin 2000.

[FLM] Frenkel, I. B., Lepowsky, J., Meurman, A., Vertex Operator Algebras and the Monster, Pure and Appl. Math., vol. 134, Academic Press, Boston, 1988.

[G] Gaitsgory, D., Notes on 2d conformal field theory and string theory, Quantum fields and strings: a course for mathematicians (P. Deligne et al., ed.), vol. 2, Amer. Math. Soc., Providence RI, 1999, pp. 1017-1089.

[GKF] Gelfand, I.M. Kazhdan, D.A., Fuks, D.B., The actions of infinite-dimensional Lie algebras, Funct. Anal. Appl. 6 (1972), 9-13.

[GV] Grothendieck, A., Verdier, J.-L., Théorie des topos et cohomologie étale des schémas, SGA IV, Exp. I, Lecture Notes in Math, vol. 269, Springer, 1970.

[HL] Huang,Y.-Z., Lepowsky, J., On the $\mathcal{D}$-module and the formal variable approachs to vertex algebras, in Topics in geometry, Birkhäuser, 1996, pp. 175-202.

[K] Kac, V., Vertex algebras for beginners, University Lecture Series, vol. 10, Amer. Math. Soc., Providence RI, 1997.

[Kap] Kapranov, M., Double affine Hecke algebras and 2-dimensional local fields, Jour. AMS 14 (2001), 239-262.

[Kat] Kato, K., Existence theorem for higher local class field theory, math.AG/0012150.

[KT] Kashiwara, M., Tanisaki, T., Kazhdan-Lusztig Conjecture for Symmetrizable KacMoody Lie Algebra. II Intersection Cohomologies of Schubert varieties, Operator algebras, unitary representations, enveloping algebras, and invariant theory (Paris, 1989), Progr. Math., vol. 92, Birkhäuser, 1990, pp. 159-195.

[L] Leites, D. A., Introduction to the theory of supermanifolds. (Russian), Uspekhi Mat. Nauk 35 (1980), 3-57.

[MSV] Malikov, A., Schechtman, V., Vaintrob, A., Chiral De Rham complex, Comm. Math. Phys. 204 (1999), 439-473.

[P] Parshin, A.N., Higher-dimensional local fields and L-functions, math.AG/0012151. 
[TT] Thomason, R.W., Trobaugh, T., Higher algebraic K-theory of schemes and of derived categories, Grothendieck Festschrift (P. Cartier et al. Eds.) vol. III, Progr. Math., vol. 88, Birkhäuser, 1990, pp. 247-435.

Mikhail Kapranov

Department of Mathematics

University of Toronto

100 St. George St.

Toronto, Ontario M5S 3G3

Canada

email: kapranov@math.toronto.edu
Eric Vasserot

Département de Mathématiques

Université de Cergy-Pontoise

2 Av. A. Chauvin

95302 Cergy-Pontoise Cedex

France

email: eric.vasserot@math.u-cergy.fr 